\title{
Spectroscopic Studies on Hydrazine-Boranes, Key Compounds for Chemical Hydrogen Storage
}

\author{
Javier Torres Escalona ${ }^{1,2}$, Jean-Claude Guillemin ${ }^{2 *}$, Clovis Darrigan ${ }^{1}$, Anna Chrostowska ${ }^{1 *}$ \\ ${ }^{1}$ CNRS / Université de Pau et des Pays de l'Adour / E2S UPPA, Institut des Sciences \\ Analytiques et de Physicochimie pour l'Environnement et les Matériaux, UMR5254, \\ 64000, Pau, France. \\ e-mail: anna.chrostowska@univ-pau.fr \\ ${ }^{2}$ Univ Rennes, Ecole Nationale Supérieure de Chimie de Rennes, CNRS, ISCR - UMR6226, \\ F-35000 Rennes, France. \\ e-mail: jean-claude.guillemin@ensc-rennes.fr
}

\section{Supporting information}

Experimental details fist of compounds 


\section{List of compounds}

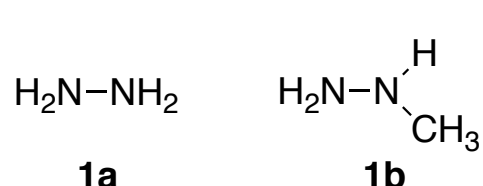

$\mathrm{H}_{3} \mathrm{~B}^{\stackrel{\mathrm{N}}{\mathrm{N}}-\mathrm{NH}_{2}}$

$2 a$

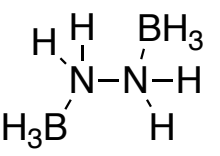

$3 a$<smiles>[B]N</smiles>

2b<smiles>CN(C)N</smiles><smiles>BN(C)N</smiles>

2c<smiles>[B]N([B])N(C)C</smiles>

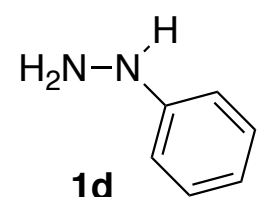<smiles>[B]NCc1ccccc1</smiles>

2d

Chart 1 


\section{Experimental details}

\section{Materials}

Hydrazine (1.0 mol/L in THF), methylhydrazine, 1,1-dimethylhydrazine, phenylhydrazine and borane-dimethylsulfide complex were purchased from Aldrich and used without further purification. Phenylhydrazine monoborane was prepared as previously reported. ${ }^{1}$

Mono- and bisborane complexes of hydrazine derivatives were synthesized in dichloromethane by mixing the free hydrazine derivative with borane-dimethylsulfide complex in dichloromethane (Scheme 1).

$$
\begin{aligned}
& \mathrm{H}_{2} \mathrm{~N}-\mathrm{NRR} \text { ' (in excess) }+\mathrm{BH}_{3} \longrightarrow \mathrm{H}_{2} \mathrm{~N}-\stackrel{\text { N }}{\mathrm{H}} \\
& \text { 1a-1c } \mathrm{R}=\mathrm{H}, \mathrm{Me} \text { 2a-2c } \mathrm{BH}_{3} \\
& \mathrm{R}=\mathrm{H}, \mathrm{Me} \stackrel{\text { 2a-2c }}{\mid \begin{array}{c}
\mathrm{BH}_{3} \\
(\text { excess })
\end{array}}
\end{aligned}
$$

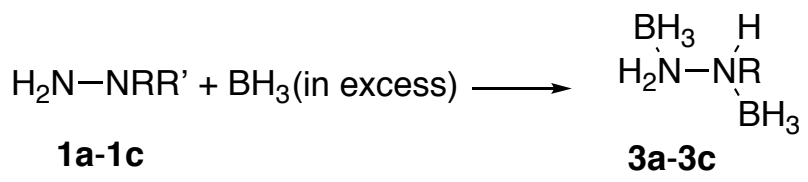

$$
\begin{aligned}
& \mathrm{H}_{2} \mathrm{~N}-\mathrm{NHPh}+\mathrm{BH}_{3} \longrightarrow \stackrel{\mathrm{H}_{3} \mathrm{~B}}{\mathrm{H}_{2}} \mathrm{~N}-\mathrm{NH}^{\mathrm{Ph}} \\
& \text { 1d 2d }
\end{aligned}
$$

Scheme 1: Synthesis of a) mono- and b) bisborane derivatives, with $R=H-, R^{\prime}=H, H_{3} C$, Ph and $\mathrm{R}=\mathrm{CH}_{3}, \mathrm{R}^{\prime}=\mathrm{H}, \mathrm{H}_{3} \mathrm{C}$.

\section{Hydrazine-monoborane derivatives}

\section{General procedure for the synthesis of hydrazine monoborane derivatives}

In a three-neck round-bottom flask under nitrogen, borane-dimethylsulfide complex (9 mmol) was added dropwise to a cooled $\left(-30^{\circ} \mathrm{C}\right)$ solution of hydrazine $(10 \mathrm{mmol})$ in dry dichloromethane $(2.5 \mathrm{~mL})$. At the end of the addition, the reaction mixture was allowed to warm to room temperature and was stirred for 30 minutes. The solvent and low boiling 
compounds were removed in vacuo at room temperature. The crude hydrazine monoborane was then washed with pentane and dried in vacuo at room temperature. Yield: $96 \%$.

\section{Hydrazine bisborane derivatives}

In a three-neck round-bottom flask under nitrogen, borane-dimethylsulfide complex (15 mmol) was added dropwise to a cooled $\left(-30^{\circ} \mathrm{C}\right)$ solution of the hydrazine $(5 \mathrm{mmol})$ in dry dichloromethane $(5 \mathrm{~mL})$. The reaction mixture was allowed to warm to room temperature and was stirred for 30 minutes. The solvent and low boiling compounds were removed in vacuo at room temperature; the crude hydrazine bisborane complex was washed with pentane and then dried in vacuo at room temperature. Yield: $95 \%$.

\section{NMR data}

\section{Hydrazine 1a}

${ }^{1} \mathrm{H}$ NMR (DMSO-d6, $400 \mathrm{MHz}$ ) $\delta 3.41$ (s brd, 4H, $\mathrm{NH}_{2}$ ).

\section{Hydrazine-monoborane 1b}

${ }^{1} \mathrm{H}$ NMR (DMSO-d6, $400 \mathrm{MHz}$ ) $\delta 1.33$ (q brd, $3 \mathrm{H},{ }^{1} \mathrm{~J}_{\mathrm{BH}}=95 \mathrm{~Hz}, \mathrm{BH}_{3}$ ) ; 3.44 (s brd, $2 \mathrm{H}, \mathrm{NH}_{2}$ ) ; 6.48 (s brd, $2 \mathrm{H}, \mathrm{NH}_{2} \mathrm{~B}$ ).

${ }^{11} \mathrm{~B} N M R(D M S O-d 6,128 \mathrm{MHz}) \delta-18.4\left(q,{ }^{1} \mathrm{~J}_{\mathrm{BH}}=95 \mathrm{~Hz}, \mathrm{BH}_{3}\right)$.

The chemical shift at down-field ( $\delta 6.42 \mathrm{ppm}$ ) corresponds to 2 hydrogen atoms on a complexed nitrogen.

\section{Hydrazine-bisborane 1c}

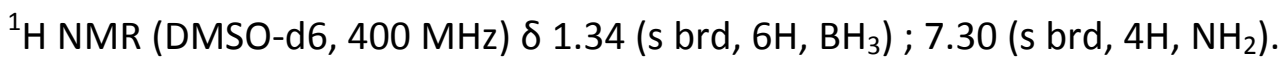

${ }^{11}$ B NMR (DMSO-d6, $\left.128 \mathrm{MHz}\right) \delta-18.9\left(\mathrm{q},{ }^{1} \mathrm{~J}_{\mathrm{BH}}=96 \mathrm{~Hz}, \mathrm{BH}_{3}\right)$.

\section{Methylhydrazine 2a}

${ }^{1} \mathrm{H}$ NMR (DMSO-d6, $\left.400 \mathrm{MHz}\right) \delta 2.38\left(\mathrm{~d}, 3 \mathrm{H},{ }^{3} \mathrm{~J}_{\mathrm{HH}}=8 \mathrm{~Hz}, \mathrm{CH}_{3}\right) ; 3.30$ (s brd, $3 \mathrm{H}, \mathrm{NHNH}_{2}$ ).

${ }^{13} \mathrm{C}$ NMR (DMSO-d6, $\left.100 \mathrm{MHz}\right) \delta 42.8\left(\mathrm{q},{ }^{1} \mathrm{~J}_{\mathrm{CH}}=131 \mathrm{~Hz}, \mathrm{CH}_{3}\right)$. 


\section{Methylhydrazine-monoborane $2 \mathrm{~b}$}

${ }^{1} \mathrm{H}$ NMR (DMSO-d6, $\left.400 \mathrm{MHz}\right) \delta 1.38$ (q brd, 3H, $\left.{ }^{1} J_{\mathrm{BH}}=96 \mathrm{~Hz}, \mathrm{BH}_{3}\right) ; 2.40\left(\mathrm{~d}, 3 \mathrm{H},{ }^{3} \mathrm{~J}_{\mathrm{HH}}=8 \mathrm{~Hz}\right.$, $\mathrm{CH}_{3}$ ); 3.93 (s brd, $2 \mathrm{H},-\mathrm{NH}_{2}$ ) ; 7.33 (s brd, $1 \mathrm{H},-\mathrm{NH}-$ ).

${ }^{13} \mathrm{C}$ NMR (DMSO-d6, $\left.100 \mathrm{MHz}\right) \delta 46.2\left(\mathrm{q},{ }^{1} \mathrm{~J}_{\mathrm{CH}}=142 \mathrm{~Hz}, \mathrm{CH}_{3}\right)$.

${ }^{11}$ B NMR (DMSO-d6, $\left.128 \mathrm{MHz}\right) \delta-13.7\left(\mathrm{q},{ }^{1} J_{\mathrm{BH}}=96 \mathrm{~Hz}, \mathrm{BH}_{3}\right)$.

The chemical shift at $3.93 \mathrm{ppm}$ with an intensity of 2 corresponds to 2 hydrogen atoms on a free nitrogen, and the signal at down-field $(\delta 7.33 \mathrm{ppm})$ with an intensity of 1 corresponds to 1 hydrogen atom on a complexed nitrogen.

\section{Methylhydrazine-bisborane 2c}

${ }^{1} \mathrm{H}$ NMR (DMSO-d6, $\left.400 \mathrm{MHz}\right) \delta 1.39$ (s brd, $\left.6 \mathrm{H}, \mathrm{BH}_{3}\right) ; 2.56\left(\mathrm{~d}, 3 \mathrm{H},{ }^{3} \mathrm{~J}_{\mathrm{HH}}=8 \mathrm{~Hz}, \mathrm{CH}_{3}\right.$ ) ; 7.5-7.7 ( $\mathrm{AB}$ system brd, $\left.2 \mathrm{H}, \mathrm{NH}_{2}\right) ; 7.76(\mathrm{~s}, 1 \mathrm{H},-\mathrm{NH})$.

${ }^{13} \mathrm{C}$ NMR (DMSO-d6, $100 \mathrm{MHz}$ ) $\delta 40.7$ (q, ${ }^{1} \mathrm{~J}_{\mathrm{CH}}=142 \mathrm{~Hz}, \mathrm{CH}_{3}$ ).

${ }^{11} \mathrm{~B} N M R(D M S O-d 6,128 \mathrm{MHz}) \delta-13.8\left(q,{ }^{1} J_{\mathrm{BH}}=96 \mathrm{~Hz}, \mathrm{BH}_{3}\right) ;-20.9\left(q,{ }^{1} J_{\mathrm{BH}}=96 \mathrm{~Hz}, \mathrm{BH}_{3}\right)$.

\section{1,1-dimethylhydrazine 3a}

${ }^{1} \mathrm{H} \mathrm{NMR}\left(\mathrm{CDCl}_{3}, 400 \mathrm{MHz}\right) \delta 2.34\left(\mathrm{~s}, 3 \mathrm{H}, \mathrm{CH}_{3}\right) ; 2.93$ (s brd, $\left.2 \mathrm{H}, \mathrm{NH}_{2}\right)$.

${ }^{13} \mathrm{C} \mathrm{NMR}\left(\mathrm{CDCl}_{3}, 100 \mathrm{MHz}\right) \delta 51.5\left(\mathrm{q},{ }^{1} \mathrm{~J}_{\mathrm{CH}}=133.4 \mathrm{~Hz}, \mathrm{CH}_{3}\right)$.

\section{1,1-dimethylhydrazine-monoborane 3b}

${ }^{1} \mathrm{H}$ NMR (DMSO-d6, $400 \mathrm{MHz}$ ) $\delta 1.69$ (q brd, 3H, $\left.{ }^{1}{ }^{J_{\mathrm{BH}}}=96 \mathrm{~Hz}, \mathrm{BH}_{3}\right) ; 2.72\left(\mathrm{~s}, 6 \mathrm{H}, \mathrm{CH}_{3}\right.$ ) ; 4.55 (s brd, $2 \mathrm{H},-\mathrm{NH}_{2}$ ).

${ }^{13} \mathrm{C}$ NMR (DMSO-d6, $\left.100 \mathrm{MHz}\right) \delta 56.1\left(\mathrm{q},{ }^{1} \mathrm{~J}_{\mathrm{CH}}=139.6 \mathrm{~Hz}, \mathrm{CH}_{3}\right)$.

${ }^{11} \mathrm{~B} N M R(D M S O-d 6,128 \mathrm{MHz}) \delta-7.4\left(\mathrm{q},{ }^{1} \mathrm{~J}_{\mathrm{BH}}=96 \mathrm{~Hz}, \mathrm{BH}_{3}\right)$.

The chemical shift at $\delta 4.55 \mathrm{ppm}$ with an intensity of 2 corresponds to 2 hydrogen atoms on a free nitrogen.

\section{1,1-dimethylhydrazine-bisborane 3c}

${ }^{1} \mathrm{H}$ NMR (DMSO-d6, $400 \mathrm{MHz}$ ) $\delta 1.47$ (q brd, 6H, $\left.{ }^{1} J_{\mathrm{BH}}=101 \mathrm{~Hz}, \mathrm{BH}_{3}\right) ; 2.75\left(\mathrm{~s}, 6 \mathrm{H}, \mathrm{CH}_{3}\right.$ ) ; 8.02 (s brd, $2 \mathrm{H},-\mathrm{NH}_{2}$ ).

${ }^{13} \mathrm{C}$ NMR (DMSO-d6, $\left.100 \mathrm{MHz}\right) \delta 49.7\left(\mathrm{q},{ }^{1} \mathrm{~J}_{\mathrm{CH}}=142 \mathrm{~Hz}, \mathrm{CH}_{3}\right.$ ). 
${ }^{11} \mathrm{~B}$ NMR (DMSO-d6, $\left.\left.128 \mathrm{MHz}\right) \delta-6.0\left(\mathrm{q},{ }^{1} J_{\mathrm{BH}}=102 \mathrm{~Hz}, \mathrm{BH}\right)_{3}\right) ;-23.0\left(\mathrm{q},{ }^{1} J_{\mathrm{BH}}=101 \mathrm{~Hz}, \mathrm{BH}_{3}\right)$.

\section{Phenylhydrazine 4a}

${ }^{1} \mathrm{H}$ NMR (DMSO-d6, $\left.400 \mathrm{MHz}\right) \delta 3.96$ (s brd, $2 \mathrm{H},-\mathrm{NH}_{2}$ ); $6.66\left(\mathrm{t}, 1 \mathrm{H},{ }^{3} \mathrm{~J}_{\mathrm{HH}}=8 \mathrm{~Hz}, \mathrm{H}_{\text {para- }}\right.$ ); 6.69 (s

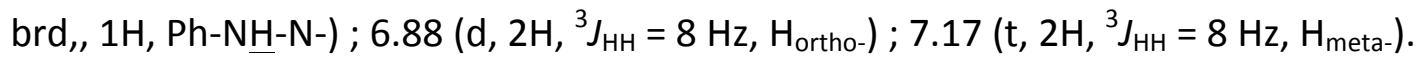

${ }^{13} \mathrm{C}$ NMR (DMSO-d6, $100 \mathrm{MHz}$ ) $\delta 111.9$ (d, ${ }^{1} \mathrm{~J}_{\mathrm{CH}}=156 \mathrm{~Hz}, \mathrm{C}_{\text {ortho-}}$ ) ; 117.2 (d, ${ }^{1} J_{\mathrm{CH}}=159 \mathrm{~Hz}, \mathrm{C}_{\text {para- }}$ ) ; $128.9\left(\mathrm{~d},{ }^{1} \mathrm{~J}_{\mathrm{CH}}=155 \mathrm{~Hz}, \mathrm{C}_{\text {meta- }}\right) ; 152.8\left(\mathrm{~s},-\underline{\mathrm{C}}_{(\mathrm{i})}-\mathrm{NH}-\right)$.

\section{Phenylhydrazine-monoborane 4b}

${ }^{1} \mathrm{H}$ NMR (DMSO-d6, $400 \mathrm{MHz}$ ) $\delta 1.51$ (s brd, 3H, BH $) ; 6.55$ (s brd, $\left.1 \mathrm{H}, \mathrm{NH}\right) ; 6.76\left(\mathrm{t}, 1 \mathrm{H},{ }^{3} \mathrm{~J}_{\mathrm{HH}}=8\right.$ $\mathrm{Hz}, \mathrm{H}_{\text {para- }}$ ); 6.90 (d, $2 \mathrm{H},{ }^{3} \mathrm{~J}_{\mathrm{HH}}=8 \mathrm{~Hz}, \mathrm{H}_{\text {ortho-) }}$; 7.15 (t, $2 \mathrm{H},{ }^{3} \mathrm{~J}_{\mathrm{HH}}=8 \mathrm{~Hz}, \mathrm{H}_{\text {meta-)}}$ ); 7.56 (s brd, $, 2 \mathrm{H}, \mathrm{NH}_{2}$ ). ${ }^{13} \mathrm{C}$ NMR (DMSO-d6, $100 \mathrm{MHz}$ ) $\delta 113.5$ (d, ${ }^{1} \mathrm{~J}_{\mathrm{CH}}=156 \mathrm{~Hz}, \mathrm{C}_{\text {ortho-}}$ ) ; 119.4 (d, ${ }^{1} J_{\mathrm{CH}}=160 \mathrm{~Hz}, \mathrm{C}_{\text {para- }}$ ) ; $128.5\left(\mathrm{~d},{ }^{1} J_{\mathrm{CH}}=155 \mathrm{~Hz}, \mathrm{C}_{\text {meta- }}\right) ; 147.8\left(\mathrm{~s},-\underline{\mathrm{C}}_{(\mathrm{i})}-\mathrm{NH}-\right)$.

${ }^{11} \mathrm{~B} N M R(D M S O, 128 \mathrm{MHz}) \delta-19.3\left(q,{ }^{1} \mathrm{~J}_{\mathrm{BH}}=98 \mathrm{~Hz}, \mathrm{BH}_{3}\right)$.

The chemical shift at down-field ( $\delta 7.56 \mathrm{ppm})$ with an intensity of 2 corresponds to 2 hydrogen atoms on a complexed nitrogen. 


\section{Computational details of optimized geometry}

\section{Hydrazines}

\section{Hydrazine (1a) \\ Neutral, ground state, CAM-B3LYP/6-311G $(d, p)$}

Atomic coordinates and value of zero point energy:

$\begin{array}{rrrr}\mathrm{H} & -0.2351220 & 1.1013280 & 0.8343270 \\ \mathrm{H} & 0.9281460 & 1.0258430 & -0.3227300 \\ \mathrm{H} & 0.2351220 & -1.1013280 & 0.8343270 \\ \mathrm{H} & -0.9281460 & -1.0258430 & -0.3227300 \\ \mathrm{~N} & 0.0000000 & 0.7115760 & -0.0730850 \\ \mathrm{~N} & 0.0000000 & -0.7115760 & -0.0730850\end{array}$

$\mathrm{ZPE}=-111.793865 \mathrm{Ha}$

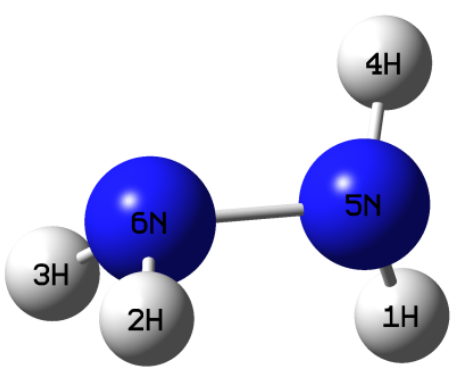

Geometrical parameters [bond distances (R) in $\AA$, angles (A) and dihedral (D) in degrees] :

$\begin{array}{lr}R(1,5) & 1.0152 \\ R(2,5) & 1.0112 \\ R(3,6) & 1.0152 \\ R(4,6) & 1.0112 \\ R(5,6) & 1.4232 \\ A(1,5,2) & 108.2966 \\ A(1,5,6) & 112.5770 \\ A(2,5,6) & 108.1065 \\ A(3,6,4) & 108.2966 \\ A(3,6,5) & 112.5770 \\ A(4,6,5) & 108.1065 \\ D(1,5,6,3) & -29.0532 \\ D(1,5,6,4) & 90.5280 \\ D(2,5,6,3) & 90.5280 \\ D(2,5,6,4) & -149.8908\end{array}$


Methylhydrazine (1b)

Neutral, ground state, CAM-B3LYP/6-311G (d,p)

Atomic coordinates and value of zero point energy:

\section{Conformer}

Form1

$-151.055322$

Energy

( $\mathrm{Ha})$

$\triangle E$

$(\mathrm{kcal} / \mathrm{mol})$

$$
3.46
$$

0.00
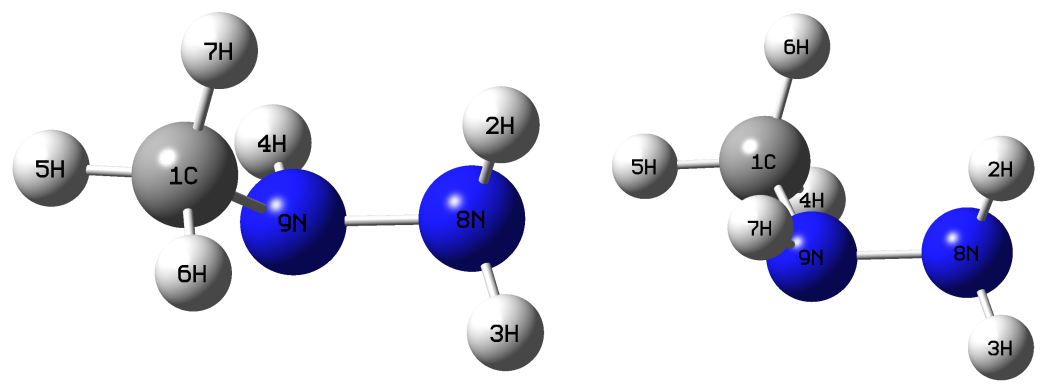

Form 2: $\quad \mathrm{ZPE}=-151.060841 \mathrm{Ha}$

$\begin{array}{lrrr}\mathrm{C} & -1.16974300 & -0.23909000 & -0.00109400 \\ \mathrm{H} & 1.12252400 & -0.90827300 & 0.72441400 \\ \mathrm{H} & 1.49826200 & -0.55744500 & -0.83633100 \\ \mathrm{H} & 0.02423900 & 1.31732700 & 0.55674300 \\ \mathrm{H} & -2.04839300 & 0.40808100 & -0.04747500 \\ \mathrm{H} & -1.22610800 & -0.94133500 & -0.83668200 \\ \mathrm{H} & -1.21597000 & -0.82182800 & 0.93488000 \\ \mathrm{~N} & 1.23719600 & -0.14878200 & 0.05280800 \\ \mathrm{~N} & 0.02907600 & 0.56849900 & -0.12266400\end{array}$

Geometrical parameters [bond distances

(D) in degrees]:

(R) in $\AA$, angles (A) and dihedral

$\begin{array}{lr}R(1,5) & 1.0922 \\ R(1,6) & 1.0929 \\ R(1,7) & 1.1035 \\ R(1,9) & 1.4506 \\ R(2,8) & 1.0203 \\ R(3,8) & 1.0128 \\ R(4,9) & 1.0111 \\ R(8,9) & 1.4159 \\ A(5,1,6) & 107.8636 \\ A(5,1,7) & 108.3732\end{array}$




$\begin{array}{lr}A(5,1,9) & 109.3535 \\ A(6,1,7) & 107.8779 \\ A(6,1,9) & 109.6496 \\ A(7,1,9) & 113.5595 \\ A(2,8,3) & 107.8478 \\ A(2,8,9) & 111.2701 \\ A(3,8,9) & 108.3940 \\ A(1,9,4) & 110.6128 \\ A(1,9,8) & 114.3769 \\ A(4,9,8) & 107.2164 \\ D(5,1,9,4) & 53.0539 \\ D(5,1,9,8) & 174.2262 \\ D(6,1,9,4) & 171.1299 \\ D(6,1,9,8) & -67.6977 \\ \text { D }(7,1,9,4) & -68.1191 \\ \text { D }(7,1,9,8) & 53.0533 \\ \text { D }(2,8,9,1) & -32.9588 \\ \text { D }(2,8,9,4) & 90.0692 \\ \text { D }(3,8,9,1) & 85.4693 \\ \text { D }(3,8,9,4) & -151.5027\end{array}$


Atomic coordinates and value of zero point energy:

Conformer

Total

Energy

( $\mathrm{Ha})$

$\Delta \mathrm{E}$

$(\mathrm{kcal} / \mathrm{mol})$
Form 1

$-190.323473$

3.24

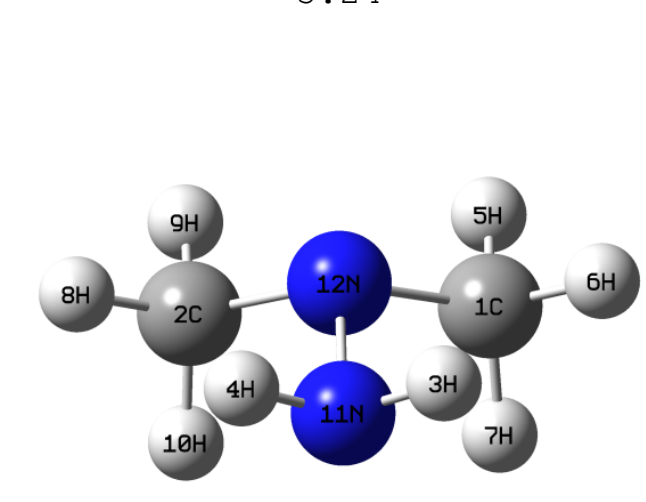

Form2

$-190.328629$

0.00

Image

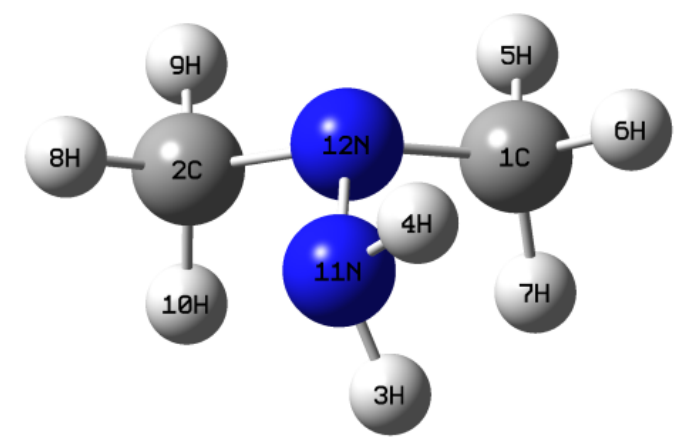

Form 2: $\mathrm{ZPE}=-190.328629 \mathrm{Ha}$

$\begin{array}{lr}\mathrm{C} & -1.15833350750 \\ \mathrm{C} & 0.02645008550 \\ \mathrm{H} & 1.13929918940 \\ \mathrm{H} & 1.39533115960 \\ \mathrm{H} & -2.05440705970 \\ \mathrm{H} & -1.14408355820 \\ \mathrm{H} & -1.22822962950 \\ \mathrm{H} & 0.92713465330 \\ \mathrm{H} & -0.84879903340 \\ \mathrm{H} & 0.00437366180 \\ \mathrm{~N} & 1.22566508010 \\ \mathrm{~N} & 0.02549946870\end{array}$

Geometrical parameters (D) in degrees] :

$\begin{array}{rr}-0.23832423790 & -0.02046452820 \\ 1.64842733290 & 0.88647504670 \\ -0.86326936640 & 0.73719595470 \\ -0.62160137980 & -0.87192526900 \\ 0.37345605760 & -0.14173616930 \\ -0.98214970190 & -0.81919387820 \\ -0.76860372470 & 0.94764274150 \\ 2.24711325440 & 0.76538816960 \\ 2.28532539050 & 0.74431472930 \\ 1.25604066020 & 1.91895523120 \\ -0.14984461960 & 0.00830693420 \\ 0.59437919460 & -0.10780353260\end{array}$

[bond distances

(R) in $\AA$, angles

(A) and dihedral
$R(1,5)$
1.092
$\mathrm{R}(1,6)$
1.092
$\mathrm{R}(1,7)$
1.106
$\mathrm{R}(1,12)$
1.450
$\mathrm{R}(2,8)$
1.088
$R(2,9)$
1.092
$\mathrm{R}(2,10)$
1.105 


\begin{tabular}{|c|c|}
\hline$R(2,12)$ & 1.449 \\
\hline$R(3,11)$ & 1.024 \\
\hline $\mathrm{R}(4,11)$ & 1.013 \\
\hline$R(11,12)$ & 1.417 \\
\hline$A(5,1,6)$ & 108.137 \\
\hline$A(5,1,7)$ & 108.302 \\
\hline$A(5,1,12)$ & 109.975 \\
\hline$A(6,1,7)$ & 108.337 \\
\hline$A(6,1,12)$ & 109.670 \\
\hline$A(7,1,12)$ & 112.312 \\
\hline$A(8,2,9)$ & 109.153 \\
\hline$A(8,2,10)$ & 108.417 \\
\hline$A(8,2,12)$ & 108.928 \\
\hline$A(9,2,10)$ & 108.233 \\
\hline$A(9,2,12)$ & 109.542 \\
\hline$A(10,2,12)$ & 112.514 \\
\hline $\mathrm{A}(3,11,4)$ & 107.958 \\
\hline$A(3,11,12)$ & 110.669 \\
\hline$A(4,11,12)$ & 108.377 \\
\hline$A(1,12,2)$ & 112.145 \\
\hline$A(1,12,11)$ & 112.642 \\
\hline$A(2,12,11)$ & 108.983 \\
\hline$D(5,1,12,2)$ & 58.129 \\
\hline$D(5,1,12,11)$ & -178.485 \\
\hline$D(6,1,12,2)$ & 176.924 \\
\hline$D(6,1,12,11)$ & -59.691 \\
\hline$D(7,1,12,2)$ & -62.556 \\
\hline$D(7,1,12,11)$ & 60.829 \\
\hline $\mathrm{D}(8,2,12,1)$ & -179.225 \\
\hline$D(8,2,12,11)$ & 55.358 \\
\hline $\mathrm{D}(9,2,12,1)$ & -59.900 \\
\hline$D(9,2,12,11)$ & 174.683 \\
\hline$D(10,2,12,1)$ & 60.532 \\
\hline$D(10,2,12,11)$ & -64.885 \\
\hline$D(3,11,12,1)$ & -41.381 \\
\hline$D(3,11,12,2)$ & 83.746 \\
\hline $\mathrm{D}(4,11,12,1)$ & 76.819 \\
\hline $\mathrm{D}(4,11,12,2)$ & -158.054 \\
\hline
\end{tabular}


Phenylhydrazine (1d)

Neutral, ground state, CAM-B3LYP/6-311G(d,p)

Atomic coordinates and value of zero point energy:

$\begin{array}{lrrr}\mathrm{C} & -0.457150 & 0.270142 & -0.068202 \\ \mathrm{C} & -0.020673 & -1.054968 & -0.072937 \\ \mathrm{C} & 0.498023 & 1.292052 & -0.006664 \\ \mathrm{C} & 1.336686 & -1.343197 & -0.016334 \\ \mathrm{C} & 1.845206 & 0.991141 & 0.048209 \\ \mathrm{C} & 2.279256 & -0.330463 & 0.044838 \\ \mathrm{H} & -2.038608 & 1.531485 & 0.153188 \\ \mathrm{H} & -2.968102 & -0.933124 & -0.636714 \\ \mathrm{H} & -2.559579 & -0.877846 & 0.961348 \\ \mathrm{H} & -0.740295 & -1.860839 & -0.129500 \\ \mathrm{H} & 0.169598 & 2.326114 & -0.004931 \\ \mathrm{H} & 1.655109 & -2.379287 & -0.022223\end{array}$

$\mathrm{ZPE}=-342.688979 \mathrm{Ha}$

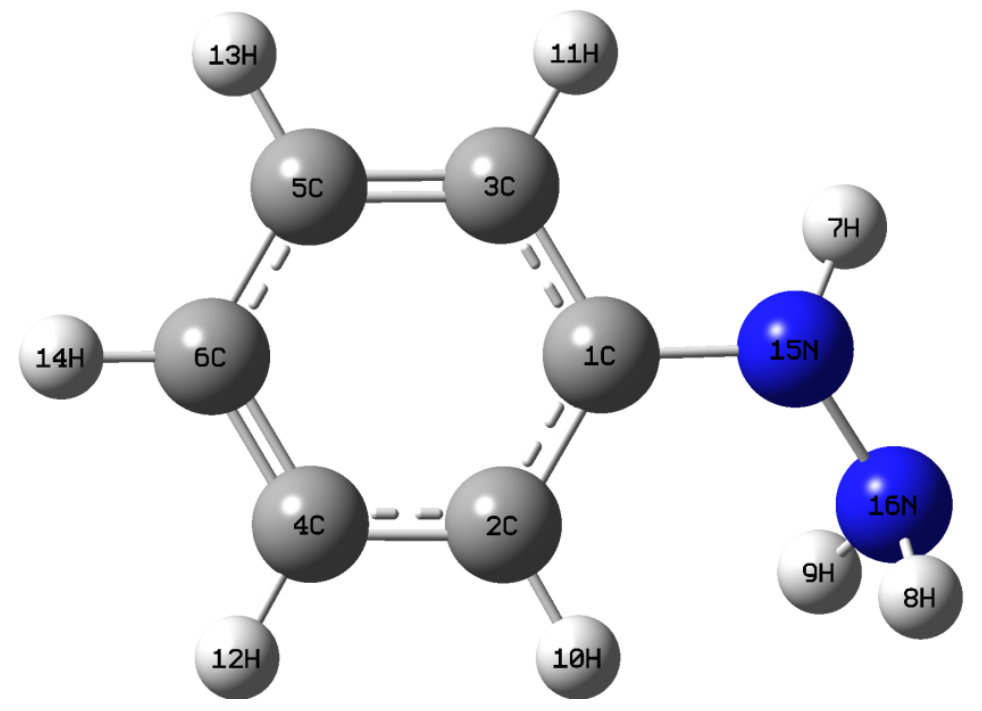

Geometrical parameters [bond distances (R) in $\AA$, angles (A) and dihedral (D) in degrees ]:

$\begin{array}{ll}R(1,2) & 1.3952 \\ R(1,3) & 1.4002 \\ R(1,15) & 1.3918 \\ R(2,4) & 1.3888 \\ R(2,10) & 1 \cdot 0819 \\ R(3,5) & 1.3815 \\ R(3,11) & 1.0850 \\ R(4,6) & 1.3849 \\ R(4,12) & 1 \cdot 0839 \\ R(5,6) & 1.3911 \\ R(5,13) & 1.0839\end{array}$




\begin{tabular}{lr} 
R $(6,14)$ & 1.0825 \\
R $(7,15)$ & 1.0076 \\
R $(8,16)$ & 1.0126 \\
R $(9,16)$ & 1.0174 \\
R $(15,16)$ & 1.3981 \\
A $(2,1,3)$ & 118.6812 \\
A $(2,1,15)$ & 122.0222 \\
A $(3,1,15)$ & 119.2686 \\
A $(1,2,4)$ & 120.1827 \\
A $(1,2,10)$ & 119.9709 \\
A $(4,2,10)$ & 119.8443 \\
A $(1,3,5)$ & 120.5325 \\
A $(1,3,11)$ & 119.2866 \\
A $(5,3,11)$ & 120.1807 \\
A $(2,4,6)$ & 121.0147 \\
A $(2,4,12)$ & 119.0308 \\
A $(6,4,12)$ & 119.9544 \\
A $(3,5,6)$ & 120.7392 \\
A $(3,5,13)$ & 119.2723 \\
A $(6,5,13)$ & 119.9882 \\
A $(4,6,5)$ & 118.8496 \\
A $(4,6,14)$ & 120.6243 \\
A $(5,6,14)$ & 120.5261 \\
A $(1,15,7)$ & 115.0707 \\
A $(1,15,16)$ & 121.5733 \\
A $(7,15,16)$ & 111.6674 \\
A $(8,16,9)$ & 108.7792 \\
A $(8,16,15)$ & 109.7800 \\
A $(9,16,15)$ & 111.3683 \\
D $(3,1,2,4)$ & 0.0506 \\
D $(3,1,2,10)$ & -179.4174 \\
D $(15,1,2,4)$ & 178.1133 \\
D $(15,1,2,10)$ & -135.4700 \\
D $(2,1,3,5)$ & -0.0010 \\
D $(2,1,3,11)$ & 179.8483 \\
D $(15,1,3,5)$ & -178.1181 \\
D $(15,1,3,11)$ & 173.1200 \\
D $(2,1,15,7)$ & 160.4305 \\
D $(2,1,15,16)$ & 20.43950 \\
D $(3,1,15,7)$ & -21.51780 \\
D $(3,1,15,16)$ & -161.5088 \\
D $(1,2,4,6)$ & -0.0341 \\
D $(1,2,4,12)$ & -179.9310 \\
D $(10,2,4,6)$ & 179.4346 \\
D $(10,2,4,12)$ & -0.4623 \\
D $(1,3,5,6)$ & -0.0664 \\
\hline
\end{tabular}




$\begin{array}{lr}\text { D }(1,3,5,13) & -179.8971 \\ \text { D }(11,3,5,6) & -179.9144 \\ \text { D }(11,3,5,13) & 0.2550 \\ \text { D }(2,4,6,5) & -0.0326 \\ \text { D }(2,4,6,14) & 179.9554 \\ \text { D }(12,4,6,5) & 179.8634 \\ \text { D }(12,4,6,14) & -0.1486 \\ \text { D }(3,5,6,4) & 0.0827 \\ \text { D }(3,5,6,14) & -179.9053 \\ \text { D }(13,5,6,4) & 179.9122 \\ \text { D }(13,5,6,14) & -0.0759 \\ \text { D }(1,15,16,8) & -81.4260 \\ \text { D }(1,15,16,9) & 39.1092 \\ \text { D }(7,15,16,8) & 137.3746 \\ \text { D }(7,15,16,9) & -102.0903\end{array}$




\section{Hydrazines borane derivatives}

Borane $\left(\mathrm{BH}_{3}\right)$

Neutral, ground state, CAM-B3LYP/6-311G(d,p)

Atomic coordinates and value of zero point energy:

$\begin{array}{cccc}\mathrm{H} & 0 . & 1.18892 & 0 . \\ \mathrm{H} & 1.02963 & -0.59446 & 0 . \\ \mathrm{H} & -1.02963 & -0.59446 & 0 . \\ \mathrm{B} & 0 . & 0 . & 0 .\end{array}$

$\mathrm{ZPE}=-26.562498 \mathrm{Ha}$

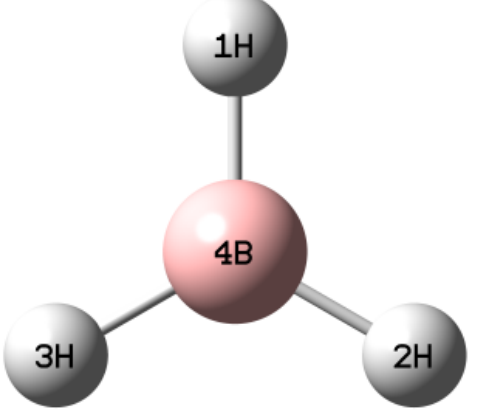

Geometrical parameters [bond distances (R) in $\AA$, angles (A) and dihedral (D) in degrees]:

$\begin{array}{lr}\mathrm{R}(1,4) & 1.1889 \\ \mathrm{R}(2,4) & 1.1889 \\ \mathrm{R}(3,4) & 1.1889 \\ \mathrm{~A}(1,4,2) & 120.0000 \\ \mathrm{~A}(1,4,3) & 120.0000 \\ \mathrm{~A}(2,4,3) & 120.0000 \\ \mathrm{D}(1,4,3,2) & 180.0000\end{array}$


Hydrazine borane (2a)

Neutral, ground state, CAM-B3LYP/6-311G(d,p)

Atomic coordinates and value of zero point energy:

Conformer

Total

Energy

( $\mathrm{Ha})$

$\triangle \mathrm{E}$

$(\mathrm{kcal} / \mathrm{mol})$
Form1

$-138.409891$

0.00

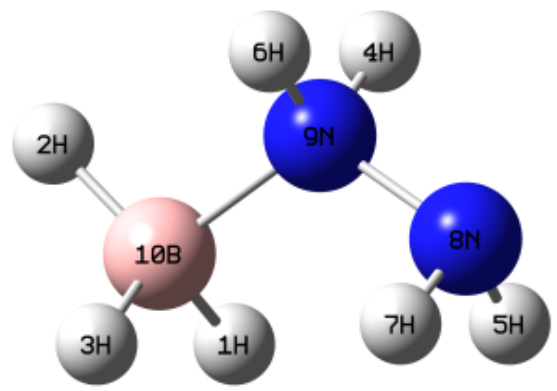

Form2

$-138.408043$

1.16

Image

Form 1: $\mathrm{ZPE}=-138.409891 \mathrm{Ha}$

$\begin{array}{lrrr}\mathrm{H} & -1.2851574208 & -0.9800026388 & -1.0112758295 \\ \mathrm{H} & -2.2371257100 & 0.4893558103 & 0.0000000000 \\ \mathrm{H} & -1.2851574208 & -0.9800026388 & 1.0112758295 \\ \mathrm{H} & 0.0647250744 & 1.1649310142 & -0.8092639242 \\ \mathrm{H} & 1.1723940735 & -0.8306660170 & -0.8011512644 \\ \mathrm{H} & 0.0647250744 & 1.1649310142 & 0.8092639242 \\ \mathrm{H} & 1.1723940735 & -0.8306660170 & 0.8011512644 \\ \mathrm{~N} & 1.2622980837 & -0.2084278451 & 0.0000000000 \\ \mathrm{~N} & 0.0256392232 & 0.5492270191 & 0.0000000000 \\ \mathrm{~B} & -1.3364759511 & -0.3166097810 & 0.0000000000\end{array}$

Geometrical parameters [bond distances

(R) in $\AA$, angles (A) and dihedral (D) in degrees ] :

$\begin{array}{ll}R(2,10) & 1.2086 \\ R(3,10) & 1.2105 \\ R(4,9) & 1.0176 \\ R(5,8) & 1.0184 \\ R(6,9) & 1.0176 \\ R(7,8) & 1.0184 \\ R(8,9) & 1.4502 \\ R(9,10) & 1.6141\end{array}$




$\begin{array}{lr}A(5,8,7) & 103.7555 \\ A(5,8,9) & 104.1222 \\ \text { A }(7,8,9) & 104.1222 \\ \text { A }(4,9,6) & 105.3607 \\ \text { A }(4,9,8) & 106.4636 \\ \text { A }(4,9,10) & 110.9081 \\ \text { A }(6,9,8) & 106.4636 \\ \text { A }(6,9,10) & 110.9081 \\ \text { A }(8,9,10) & 116.0693 \\ \text { A }(1,10,2) & 113.3944 \\ \text { A }(1,10,3) & 113.3228 \\ \text { A }(1,10,9) & 104.9602 \\ \text { A }(2,10,3) & 113.3944 \\ \text { A }(2,10,9) & 105.7256 \\ \text { A }(3,10,9) & 104.9602 \\ \text { D }(5,8,9,4) & -69.7620 \\ \text { D }(5,8,9,6) & 178.1912 \\ \text { D }(5,8,9,10) & 54.2146 \\ \text { D }(7,8,9,4) & -178.1912 \\ \text { D }(7,8,9,6) & 69.7620 \\ \text { D }(7,8,9,10) & -54.2146 \\ \text { D }(4,9,10,1) & 61.7903 \\ \text { D }(4,9,10,2) & -58.3558 \\ \text { D }(4,9,10,3) & -178.5019 \\ \text { D }(6,9,10,1) & 178.5019 \\ \text { D }(6,9,10,2) & 58.3558 \\ \text { D }(6,9,10,3) & -61.7903 \\ \text { D }(8,9,10,1) & -59.8539 \\ \text { D }(8,9,10,2) & 180.0000 \\ \text { D }(8,9,10,3) & 59.8539\end{array}$




\section{Methylhydrazine borane (2b)}

Neutral, ground state, CAM-B3LYP/6-311G(d,p)

Atomic coordinates and value of zero point energy:

\begin{tabular}{|c|c|c|c|c|c|c|}
\hline Regioisomer & Form7 & Form 8 & Form 9 & Form13 & Form 14 & Form 15 \\
\hline $\begin{array}{c}\text { Total } \\
\text { Energy (Ha) }\end{array}$ & -177.677755 & -177.675669 & -177.675587 & -177.673135 & -177.674343 & -177.6679867 \\
\hline $\begin{array}{c}\Delta \mathrm{E} \\
(\mathrm{kcal} / \mathrm{mol})\end{array}$ & 0.00 & 0.002 & 0.002 & 0.005 & 0.003 & 0.010 \\
\hline
\end{tabular}

Form 7: $\mathrm{ZPE}=-177.677755 \mathrm{Ha}$

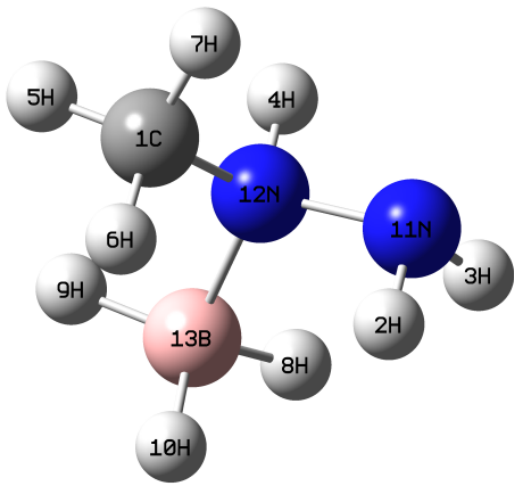

$\begin{array}{lrrr}\mathrm{C} & -1.29396 & -0.55058 & 0.09631 \\ \mathrm{H} & 0.08709 & 1.36826 & 1.06607 \\ \mathrm{H} & 1.00598 & 1.68405 & -0.21033 \\ \mathrm{H} & -0.00949 & 0.01338 & -1.36011 \\ \mathrm{H} & -1.39841 & -1.55365 & -0.31009 \\ \mathrm{H} & -1.28325 & -0.61435 & 1.18358 \\ \mathrm{H} & -2.10471 & 0.09713 & -0.23625 \\ \mathrm{H} & 2.23671 & -0.29312 & -0.33777 \\ \mathrm{H} & 1.12653 & -1.9783 & -0.31639 \\ \mathrm{H} & 1.25023 & -0.82892 & 1.34371 \\ \mathrm{~N} & 0.06414 & 1.39442 & 0.04745 \\ \mathrm{~N} & 0.00087 & -0.00213 & -0.34286 \\ \mathrm{~B} & 1.27961 & -0.86741 & 0.13352\end{array}$

Geometrical parameters [bond distances (R) in $\AA$, angles (A) and dihedral (D) in degrees ] : 


\begin{tabular}{|c|c|}
\hline $\mathrm{R}(1,5)$ & 1.0873 \\
\hline$R(1,6)$ & 1.0892 \\
\hline$R(1,7)$ & 1.0897 \\
\hline$R(1,12)$ & 1.4732 \\
\hline$R(2,11)$ & 1.0192 \\
\hline$R(3,11)$ & 1.0185 \\
\hline$R(4,12)$ & 1.0174 \\
\hline$R(8,13)$ & 1.2116 \\
\hline$R(9,13)$ & 1.2083 \\
\hline$R(10,13)$ & 1.2112 \\
\hline $\mathrm{R}(11,12)$ & 1.4514 \\
\hline $\mathrm{R}(12,13)$ & 1.6158 \\
\hline$A(5,1,6)$ & 108.6654 \\
\hline$A(5,1,7)$ & 111.2727 \\
\hline $\mathrm{A}(5,1,12)$ & 108.4487 \\
\hline$A(6,1,7)$ & 110.2897 \\
\hline$A(6,1,12)$ & 108.1038 \\
\hline$A(7,1,12)$ & 109.9791 \\
\hline $\mathrm{A}(2,11,3)$ & 103.8518 \\
\hline$A(2,11,12)$ & 104.1838 \\
\hline$A(3,11,12)$ & 104.2321 \\
\hline $\mathrm{A}(1,12,4)$ & 107.1443 \\
\hline$A(1,12,11)$ & 108.4425 \\
\hline$A(1,12,13)$ & 114.0998 \\
\hline$A(4,12,11)$ & 104.7524 \\
\hline$A(4,12,13)$ & 108.1199 \\
\hline$A(11,12,13)$ & 113.6709 \\
\hline$A(8,13,9)$ & 113.0186 \\
\hline$A(8,13,10)$ & 113.1271 \\
\hline$A(8,13,12)$ & 104.8712 \\
\hline$A(9,13,10)$ & 113.4663 \\
\hline$A(9,13,12)$ & 106.3979 \\
\hline$A(10,13,12)$ & 104.9734 \\
\hline$D(5,1,12,4)$ & 59.9502 \\
\hline$D(5,1,12,11)$ & 172.5185 \\
\hline$D(5,1,12,13)$ & -59.6891 \\
\hline$D(6,1,12,4)$ & 177.5966 \\
\hline$D(6,1,12,11)$ & -69.8351 \\
\hline $\mathrm{D}(6,1,12,13)$ & 57.9573 \\
\hline$D(7,1,12,4)$ & -61.9362 \\
\hline$D(7,1,12,11)$ & 50.6321 \\
\hline$D(7,1,12,13)$ & 178.4245 \\
\hline $\mathrm{D}(2,11,12,1)$ & 66.6035 \\
\hline$D(2,11,12,4)$ & -179.2454 \\
\hline$D(2,11,12,13)$ & -61.4328 \\
\hline$D(3,11,12,1)$ & 175.1976 \\
\hline
\end{tabular}




$\begin{array}{lr}\text { D }(3,11,12,4) & -70.6513 \\ \text { D }(3,11,12,13) & 47.1613 \\ \text { D }(1,12,13,8) & 178.4651 \\ \text { D }(1,12,13,9) & 58.4731 \\ \text { D }(1,12,13,10) & -62.0834 \\ \text { D }(4,12,13,8) & 59.3769 \\ \text { D }(4,12,13,9) & -60.6151 \\ \text { D }(4,12,13,10) & 178.8284 \\ \text { D }(11,12,13,8) & -56.4714 \\ \text { D }(11,12,13,9) & -176.4633 \\ \text { D (11,12,13,10) } & 62.9801\end{array}$


1,1-Dimethylhydrazine borane (2c)

Neutral, ground state, CAM-B3LYP/6-311G $(d, p)$

Atomic coordinates and value of zero point energy:

Regioisomer

Total

Energy (Ha)

$\triangle \mathrm{E}$

$(\mathrm{kcal} / \mathrm{mol})$
Form1

$-216.944407$

0.00

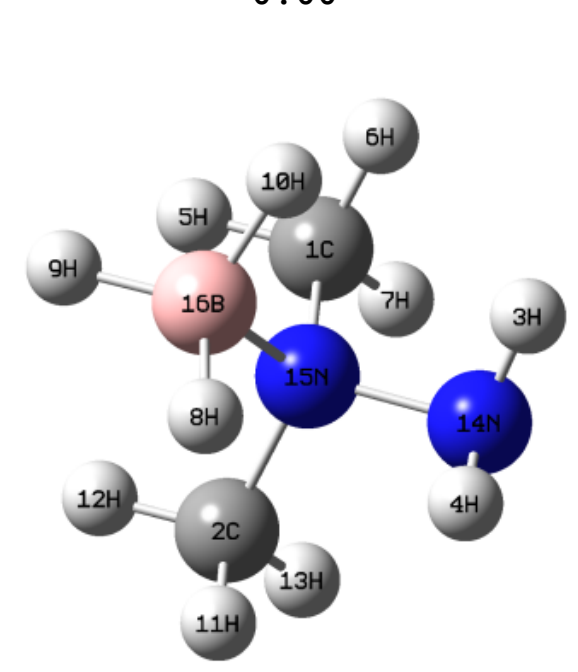

Form2

$-216.932887$

Image

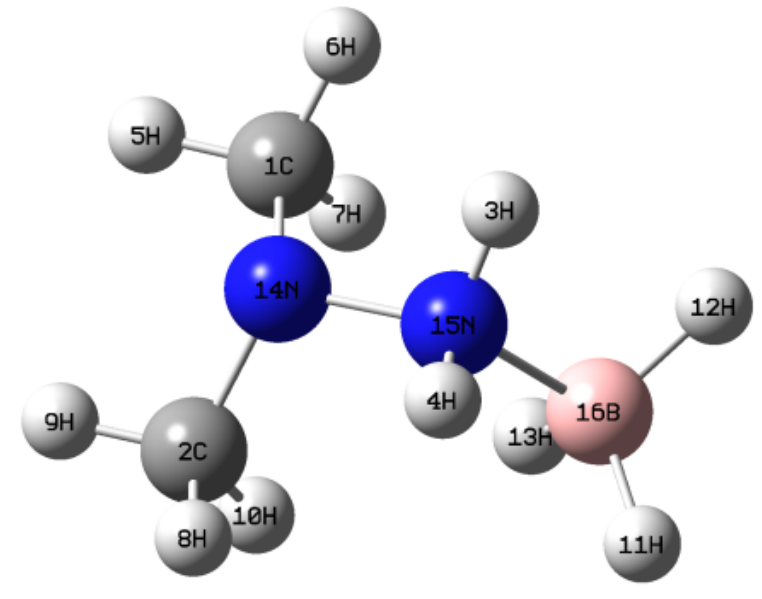

Form1: $\mathrm{ZPE}=-216.944407 \mathrm{Ha}$

$\begin{array}{rrrr}\mathrm{C} & -1.293963 & -0.550580 & 0.096312 \\ \mathrm{H} & 0.087092 & 1.368259 & 1.066067 \\ \mathrm{H} & 1.005973 & 1.684052 & -0.210330 \\ \mathrm{H} & -0.009494 & 0.013379 & -1.360109 \\ \mathrm{H} & -1.398410 & -1.553650 & -0.310090 \\ \mathrm{H} & -1.283250 & -0.614354 & 1.183577 \\ \mathrm{H} & -2.104711 & 0.097123 & -0.236246 \\ \mathrm{H} & 2.236709 & -0.293119 & -0.337766 \\ \mathrm{H} & 1.126535 & -1.978303 & -0.316389 \\ \mathrm{H} & 1.250226 & -0.828914 & 1.343708 \\ \mathrm{~N} & 0.064134 & 1.394424 & 0.047446 \\ \mathrm{~N} & 0.000873 & -0.002129 & -0.342862 \\ \mathrm{~B} & 1.279612 & -0.867411 & 0.133524\end{array}$

Geometrical parameters [bond distances

(R) in $\AA$, angles (A) and dihedral

(D) in degrees ]:
$R(1,5)$
1.0872
$\mathrm{R}(1,6)$
1.0880
$\mathrm{R}(1,7)$
1.0904
$\mathrm{R}(1,15)$
1.4768
$\mathrm{R}(2,11)$
1.0880 


\begin{tabular}{|c|c|}
\hline$R(2,12)$ & 1.0872 \\
\hline$R(2,13)$ & 1.0904 \\
\hline$R(2,15)$ & 1.4768 \\
\hline$R(3,14)$ & 1.0189 \\
\hline$R(4,14)$ & 1.0189 \\
\hline$R(8,16)$ & 1.2109 \\
\hline$R(9,16)$ & 1.2079 \\
\hline$R(10,16)$ & 1.2109 \\
\hline $\mathrm{R}(14,15)$ & 1.4554 \\
\hline$R(15,16)$ & 1.6270 \\
\hline$A(5,1,6)$ & 108.7423 \\
\hline$A(5,1,7)$ & 111.1376 \\
\hline$A(5,1,15)$ & 108.4444 \\
\hline$A(6,1,7)$ & 110.2751 \\
\hline$A(6,1,15)$ & 108.5463 \\
\hline$A(7,1,15)$ & 109.6337 \\
\hline $\mathrm{A}(11,2,12)$ & 108.7423 \\
\hline$A(11,2,13)$ & 110.2751 \\
\hline$A(11,2,15)$ & 108.5463 \\
\hline$A(12,2,13)$ & 111.1376 \\
\hline$A(12,2,15)$ & 108.4444 \\
\hline$A(13,2,15)$ & 109.6337 \\
\hline $\mathrm{A}(3,14,4)$ & 103.7812 \\
\hline$A(3,14,15)$ & 104.3101 \\
\hline$A(4,14,15)$ & 104.3101 \\
\hline$A(1,15,2)$ & 109.4270 \\
\hline$A(1,15,14)$ & 106.6118 \\
\hline$A(1,15,16)$ & 111.3121 \\
\hline$A(2,15,14)$ & 106.6118 \\
\hline$A(2,15,16)$ & 111.3121 \\
\hline $\mathrm{A}(14,15,16)$ & 111.3482 \\
\hline$A(8,16,9)$ & 113.1387 \\
\hline$A(8,16,10)$ & 112.8684 \\
\hline$A(8,16,15)$ & 104.9892 \\
\hline$A(9,16,10)$ & 113.1387 \\
\hline$A(9,16,15)$ & 106.8149 \\
\hline$A(10,16,15)$ & 104.9892 \\
\hline$D(5,1,15,2)$ & 62.2053 \\
\hline $\mathrm{D}(5,1,15,14)$ & 177.1390 \\
\hline $\mathrm{D}(5,1,15,16)$ & -61.2489 \\
\hline$D(6,1,15,2)$ & -179.8150 \\
\hline $\mathrm{D}(6,1,15,14)$ & -64.8814 \\
\hline $\mathrm{D}(6,1,15,16)$ & 56.7308 \\
\hline$D(7,1,15,2)$ & -59.3003 \\
\hline $\mathrm{D}(7,1,15,14)$ & 55.6334 \\
\hline$D(7,1,15,16)$ & 177.2455 \\
\hline
\end{tabular}




$\begin{array}{lr}\text { D }(11,2,15,1) & 179.8150 \\ \text { D }(11,2,15,14) & 64.8814 \\ \text { D }(11,2,15,16) & -56.7308 \\ \text { D }(12,2,15,1) & -62.2053 \\ \text { D }(12,2,15,14) & -177.1390 \\ \text { D }(12,2,15,16) & 61.2489 \\ \text { D }(13,2,15,1) & 59.3003 \\ \text { D }(13,2,15,14) & -55.6334 \\ \text { D }(13,2,15,16) & -177.2455 \\ \text { D }(3,14,15,1) & 67.2945 \\ \text { D }(3,14,15,2) & -175.8839 \\ \text { D }(3,14,15,16) & -54.2947 \\ \text { D }(4,14,15,1) & 175.8839 \\ \text { D }(4,14,15,2) & -67.2945 \\ \text { D }(4,14,15,16) & 54.2947 \\ \text { D }(1,15,16,8) & -178.4236 \\ \text { D }(1,15,16,9) & 61.1864 \\ \text { D }(1,15,16,10) & -59.2036 \\ \text { D }(2,15,16,8) & 59.2036 \\ \text { D }(2,15,16,9) & -61.1864 \\ \text { D (2,15,16,10) } & 178.4236 \\ \text { D (14,15,16,8) } & -59.6100 \\ \text { D (14,15,16,9) } & 180.0000 \\ \text { D (14,15,16,10) } & 59.6100\end{array}$




\section{Phenylhydrazine borane (2d)}

Neutral, ground state, CAM-B3LYP/6-311G(d,p)

Atomic coordinates and value of zero point energy:

gioisomer

Total

lergy (Ha)

$\Delta \mathrm{E}$

$\mathrm{kcal} / \mathrm{mol}$ )
Form1

$-369.289809$

4.96

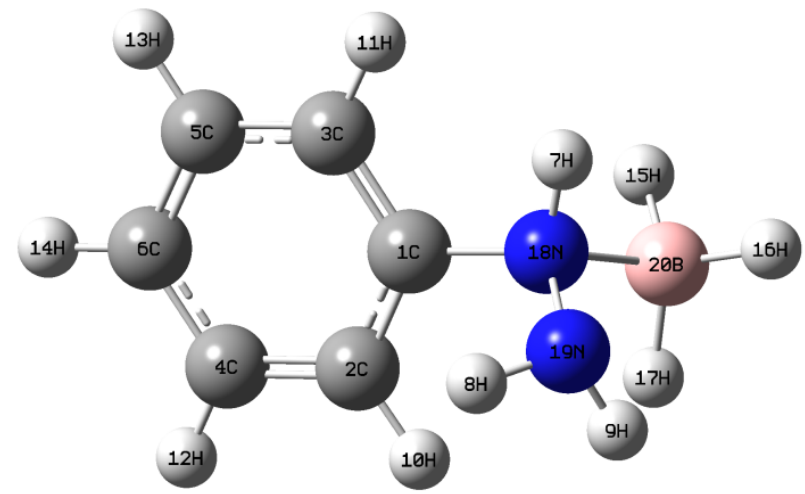

Form2

$-369.297706$

Image

Form2: ZPE $=-369.297706 \mathrm{Ha}$

$\begin{array}{lrrr}\mathrm{C} & -0.061817 & -0.213906 & -0.185238 \\ \mathrm{C} & -0.942242 & -1.289462 & -0.050697 \\ \mathrm{C} & -0.563076 & 1.084938 & -0.143002 \\ \mathrm{C} & -2.294041 & -1.063489 & 0.125658 \\ \mathrm{C} & -1.923789 & 1.298182 & 0.041635 \\ \mathrm{C} & -2.795975 & 0.232312 & 0.176779 \\ \mathrm{H} & 2.349886 & 1.177679 & -0.749192 \\ \mathrm{H} & 1.911571 & 0.988101 & 0.829787 \\ \mathrm{H} & -0.557201 & -2.302390 & -0.086422 \\ \mathrm{H} & 0.090603 & 1.938784 & -0.272079 \\ \mathrm{H} & -2.963289 & -1.908860 & 0.230790 \\ \mathrm{H} & -2.298561 & 2.314146 & 0.072940 \\ \mathrm{H} & -3.855033 & 0.404491 & 0.319073 \\ \mathrm{H} & 1.618568 & -1.379423 & -0.076236 \\ \mathrm{H} & 3.474728 & -0.953878 & 1.211502 \\ \mathrm{H} & 4.074953 & -0.661926 & -0.697417 \\ \mathrm{H} & 4.374083 & 0.779293 & 0.689679 \\ \mathrm{~N} & 1.297010 & -0.480755 & -0.412595 \\ \mathrm{~N} & 2.238818 & 0.494507 & -0.003685 \\ \mathrm{~B} & 3.702907 & -0.156747 & 0.330144\end{array}$


Geometrical parameters [bond distances (R) in $\AA$, angles $(A)$ and dihedral (D) in degrees] :

\begin{tabular}{|c|c|}
\hline$R(1,2)$ & 1.3964 \\
\hline$R(1,3)$ & 1.3929 \\
\hline $\mathrm{R}(1,18)$ & 1.4033 \\
\hline$R(2,4)$ & 1.3819 \\
\hline$R(2,9)$ & 1.0842 \\
\hline$R(3,5)$ & 1.3896 \\
\hline$R(3,10)$ & 1.0831 \\
\hline$R(4,6)$ & 1.3906 \\
\hline $\mathrm{R}(4,11)$ & 1.0833 \\
\hline$R(5,6)$ & 1.3839 \\
\hline$R(5,12)$ & 1.0833 \\
\hline$R(6,13)$ & 1.0824 \\
\hline$R(7,19)$ & 1.0173 \\
\hline$R(8,19)$ & 1.0224 \\
\hline$R(14,18)$ & 1.0120 \\
\hline$R(15,20)$ & 1.2101 \\
\hline$R(16,20)$ & 1.2040 \\
\hline$R(17,20)$ & 1.2066 \\
\hline $\mathrm{R}(18,19)$ & 1.4161 \\
\hline$R(19,20)$ & 1.6368 \\
\hline$A(2,1,3)$ & 119.2360 \\
\hline$A(2,1,18)$ & 118.6615 \\
\hline$A(3,1,18)$ & 122.0529 \\
\hline$A(1,2,4)$ & 120.2057 \\
\hline$A(1,2,9)$ & 119.5039 \\
\hline$A(4,2,9)$ & 120.2904 \\
\hline$A(1,3,5)$ & 119.9677 \\
\hline$A(1,3,10)$ & 120.9532 \\
\hline$A(5,3,10)$ & 119.0678 \\
\hline$A(2,4,6)$ & 120.6761 \\
\hline $\mathrm{A}(2,4,11)$ & 119.2821 \\
\hline$A(6,4,11)$ & 120.0406 \\
\hline$A(3,5,6)$ & 120.7919 \\
\hline$A(3,5,12)$ & 119.1101 \\
\hline$A(6,5,12)$ & 120.0969 \\
\hline$A(4,6,5)$ & 119.1204 \\
\hline$A(4,6,13)$ & 120.4150 \\
\hline$A(5,6,13)$ & 120.4646 \\
\hline $\mathrm{A}(1,18,14)$ & 115.0041 \\
\hline $\mathrm{A}(1,18,19)$ & 117.7906 \\
\hline$A(14,18,19)$ & 107.7144 \\
\hline$A(7,19,8)$ & 107.9470 \\
\hline$A(7,19,18)$ & 108.8751 \\
\hline$A(7,19,20)$ & 108.6031 \\
\hline
\end{tabular}




\begin{tabular}{|c|c|}
\hline$A(8,19,18)$ & 110.7932 \\
\hline$A(8,19,20)$ & 108.1866 \\
\hline$A(18,19,20)$ & 112.319 \\
\hline$A(15,20,16)$ & 113.797 \\
\hline$A(15,20,17)$ & 13.508 \\
\hline$A(15,20,19)$ & 104.003 \\
\hline$A(16,20,17)$ & $114.0^{-}$ \\
\hline$A(16,20,19)$ & 105.622 \\
\hline$A(17,20,19)$ & $104.457^{\circ}$ \\
\hline$D(3,1,2,4)$ & -0.1682 \\
\hline$D(3,1,2,9)$ & 179.7860 \\
\hline $\mathrm{D}(18,1,2,4)$ & -177.6601 \\
\hline $\mathrm{D}(18,1,2,9)$ & 22.9410 \\
\hline$D(2,1,3,5)$ & 0.49 \\
\hline$D(2,1,3,10)$ & -178.2756 \\
\hline $\mathrm{D}(18,1,3,5)$ & 177.895 \\
\hline$D(18,1,3,10)$ & -0.8723 \\
\hline $\mathrm{D}(2,1,18,14)$ & -25.3966 \\
\hline $\mathrm{D}(2,1,18,19)$ & -154.1020 \\
\hline $\mathrm{D}(3,1,18,14)$ & 157.1857 \\
\hline$D(3,1,18,19)$ & 28.4803 \\
\hline$D(1,2,4,6)$ & -0.2443 \\
\hline$D(1,2,4,11)$ & -179.8549 \\
\hline$D(9,2,4,6)$ & 179.8018 \\
\hline$D(9,2,4,11)$ & 0.1913 \\
\hline$D(1,3,5,6)$ & -0.4109 \\
\hline$D(1,3,5,12)$ & 179.9596 \\
\hline$D(10,3,5,6)$ & 178.3797 \\
\hline$D(10,3,5,12)$ & -124.9800 \\
\hline$D(2,4,6,5)$ & 0.3289 \\
\hline$D(2,4,6,13)$ & -179.7522 \\
\hline$D(11,4,6,5)$ & 179.9365 \\
\hline$D(11,4,6,13)$ & -0.1446 \\
\hline$D(3,5,6,4)$ & -0.0006 \\
\hline$D(3,5,6,13)$ & -179.9194 \\
\hline$D(12,5,6,4)$ & 179.6253 \\
\hline$D(12,5,6,13)$ & -0.2936 \\
\hline $\mathrm{D}(1,18,19,7)$ & -86.2956 \\
\hline$D(1,18,19,8)$ & 32.2699 \\
\hline$D(1,18,19,20)$ & 153.3865 \\
\hline $\mathrm{D}(14,18,19,7)$ & 141.6437 \\
\hline$D(14,18,19,8)$ & -99.7908 \\
\hline $\mathrm{D}(14,18,19,20)$ & 21.3258 \\
\hline $\mathrm{D}(7,19,20,15)$ & -179.5964 \\
\hline$D(7,19,20,16)$ & -59.49 \\
\hline$D(7,19,20,17)$ & 61.13 \\
\hline
\end{tabular}




$\begin{array}{lr}D(8,19,20,15) & 63.4801 \\ D(8,19,20,16) & -176.4175 \\ D(8,19,20,17) & -55.7852 \\ D(18,19,20,15) & -59.1211 \\ D(18,19,20,16) & 60.9813 \\ D(18,19,20,17) & -178.3865\end{array}$




\section{Hydrazine bisborane derivatives}

\section{Hydrazine bisborane (3a)}

Neutral, ground state, CAM-B3LYP/6-311G $(d, p)$

Atomic coordinates and value of zero point energy:

$\begin{array}{lccr}\mathrm{H} & 1.02903 & 0.48815 & 0.81376 \\ \mathrm{H} & 1.02903 & 0.48815 & -0.81376 \\ \mathrm{H} & -1.02903 & -0.48815 & 0.81376 \\ \mathrm{H} & -1.02903 & -0.48815 & -0.81376 \\ \mathrm{H} & 1.0806 & -1.9234 & -1.01153 \\ \mathrm{H} & -0.40705 & -2.84796 & 0 . \\ \mathrm{H} & 1.0806 & -1.9234 & 1.01153 \\ \mathrm{H} & -1.0806 & 1.9234 & -1.01153 \\ \mathrm{H} & 0.40705 & 2.84796 & 0 . \\ \mathrm{H} & -1.0806 & 1.9234 & 1.01153 \\ \mathrm{~N} & 0.42042 & 0.58675 & 0 . \\ \mathrm{N} & -0.42042 & -0.58675 & 0 . \\ \mathrm{B} & 0.42042 & -1.97498 & 0 . \\ \mathrm{B} & -0.42042 & 1.97498 & 0 .\end{array}$

$\mathrm{ZPE}=-165.017941 \mathrm{Ha}$

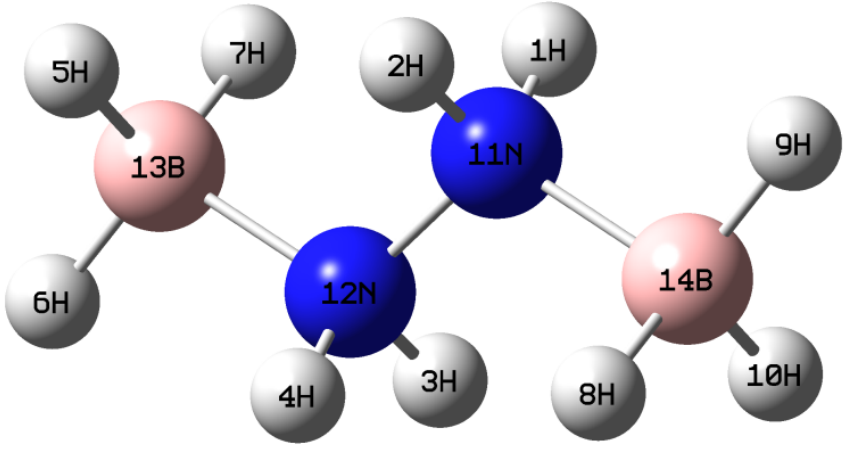

Geometrical parameters [bond distances

(R) in $\AA$, angles (A) and dihedral

(D) in degrees]:

$\begin{array}{ll}R(1,11) & 1.0209 \\ R(2,11) & 1.0209 \\ R(3,12) & 1.0209 \\ R(4,12) & 1.0209 \\ R(5,13) & 1.2090 \\ R(6,13) & 1.2028 \\ R(7,13) & 1.2090 \\ R(8,14) & 1.2090 \\ R(9,14) & 1.2028 \\ R(10,14) & 1.2090 \\ R(11,12) & 1.4436 \\ R(11,14) & 1.6230 \\ R(12,13) & 1.6230\end{array}$




\begin{tabular}{|c|c|}
\hline$A(1,11,2)$ & 105.7009 \\
\hline$A(1,11,12)$ & 105.5869 \\
\hline$A(1,11,14)$ & 113.0441 \\
\hline$A(2,11,12)$ & 105.5869 \\
\hline$A(2,11,14)$ & 113.0441 \\
\hline $\mathrm{A}(12,11,14)$ & 113.1751 \\
\hline$A(3,12,4)$ & 105.7009 \\
\hline$A(3,12,11)$ & 105.5869 \\
\hline$A(3,12,13)$ & 113.0441 \\
\hline$A(4,12,11)$ & 105.5869 \\
\hline$A(4,12,13)$ & 113.0441 \\
\hline$A(11,12,13)$ & 113.1751 \\
\hline$A(5,13,6)$ & 113.9920 \\
\hline$A(5,13,7)$ & 113.5787 \\
\hline$A(5,13,12)$ & 104.2650 \\
\hline$A(6,13,7)$ & 113.9920 \\
\hline$A(6,13,12)$ & 105.3306 \\
\hline$A(7,13,12)$ & 104.2650 \\
\hline$A(8,14,9)$ & 113.9920 \\
\hline$A(8,14,10)$ & 113.5787 \\
\hline$A(8,14,11)$ & 104.2650 \\
\hline$A(9,14,10)$ & 113.9920 \\
\hline$A(9,14,11)$ & 105.3306 \\
\hline$A(10,14,11)$ & 104.2650 \\
\hline $\mathrm{D}(1,11,12,3)$ & 68.3156 \\
\hline $\mathrm{D}(1,11,12,4)$ & 180.0000 \\
\hline $\mathrm{D}(1,11,12,13)$ & -55.8422 \\
\hline$D(2,11,12,3)$ & 180.0000 \\
\hline $\mathrm{D}(2,11,12,4)$ & -68.3156 \\
\hline $\mathrm{D}(2,11,12,13)$ & 55.8422 \\
\hline$D(14,11,12,3)$ & -55.8422 \\
\hline $\mathrm{D}(14,11,12,4)$ & 55.8422 \\
\hline $\mathrm{D}(14,11,12,13)$ & 180.0000 \\
\hline$D(1,11,14,8)$ & -179.6692 \\
\hline $\mathrm{D}(1,11,14,9)$ & 60.0177 \\
\hline$D(1,11,14,10)$ & -60.2954 \\
\hline $\mathrm{D}(2,11,14,8)$ & 60.2954 \\
\hline $\mathrm{D}(2,11,14,9)$ & -60.0177 \\
\hline $\mathrm{D}(2,11,14,10)$ & 179.6692 \\
\hline$D(12,11,14,8)$ & -59.6869 \\
\hline $\mathrm{D}(12,11,14,9)$ & 180.0000 \\
\hline $\mathrm{D}(12,11,14,10)$ & 59.6869 \\
\hline$D(3,12,13,5)$ & -179.6692 \\
\hline$D(3,12,13,6)$ & 60.0177 \\
\hline $\mathrm{D}(3,12,13,7)$ & -60.2954 \\
\hline$D(4,12,13,5)$ & 60.29 \\
\hline
\end{tabular}


D $(4,12,13,6)$

D $(4,12,13,7)$

$\mathrm{D}(11,12,13,5)$

$D(11,12,13,6)$

D $(11,12,13,7)$
$-60.0177$

179.6692

$-59.6869$

180.0000

59.6869 


\section{Methylhydrazine bisborane (3b)}

\section{Neutral, ground state, CAM-B3LYP/6-311G(d,p)}

Atomic coordinates and value of zero point energy:

$\begin{array}{lrrr}\mathrm{C} & -0.67027 & 1.42571 & 0.11251 \\ \mathrm{H} & 0.59566 & -0.33928 & 1.37297 \\ \mathrm{H} & 0.60331 & -1.54404 & 0.27403 \\ \mathrm{H} & -0.18531 & 0.00618 & -1.27513 \\ \mathrm{H} & -1.4793 & 1.81291 & -0.50119 \\ \mathrm{H} & -0.98918 & 1.43357 & 1.15404 \\ \mathrm{H} & 0.23842 & 2.00672 & -0.02654 \\ \mathrm{H} & -1.36793 & -2.03057 & -0.44859 \\ \mathrm{H} & -2.63346 & -0.47549 & -0.70477 \\ \mathrm{H} & -1.92817 & -0.91392 & 1.13984 \\ \mathrm{H} & 2.94814 & -0.75993 & 0.35487 \\ \mathrm{H} & 2.06613 & -0.27042 & -1.39439 \\ \mathrm{H} & 2.27641 & 1.11437 & 0.06532 \\ \mathrm{~N} & 0.71243 & -0.53352 & 0.37819 \\ \mathrm{~N} & -0.45385 & 0.01956 & -0.28997 \\ \mathrm{~B} & -1.73902 & -0.94761 & -0.05404 \\ \mathrm{~B} & 2.15238 & -0.05173 & -0.20659\end{array}$

$\mathrm{ZPE}=-204.284614 \mathrm{Ha}$

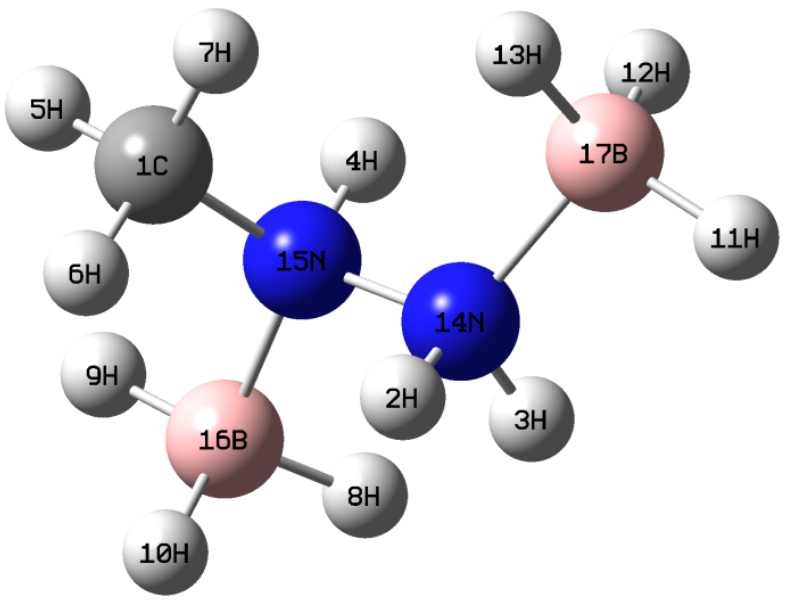

Geometrical parameters [bond distances (R) in $\AA$, angles (A) and dihedral (D) in degrees] :

$\begin{array}{ll}R(1,5) & 1.0868 \\ R(1,6) & 1.0893 \\ R(1,7) & 1.0875 \\ R(1,15) & 1.4785 \\ R(2,14) & 1.0203 \\ R(3,14) & 1.0217 \\ R(4,15) & 1.0212 \\ R(8,16) & 1.2109 \\ R(9,16) & 1.2027 \\ R(10,16) & 1.2092 \\ R(11,17) & 1.2042 \\ R(12,17) & 1.2108\end{array}$




\begin{tabular}{|c|c|}
\hline$R(13,17)$ & 1.2038 \\
\hline $\mathrm{R}(14,15)$ & 1.4535 \\
\hline $\mathrm{R}(14,17)$ & 1.6271 \\
\hline$R(15,16)$ & 1.6257 \\
\hline$A(5,1,6)$ & 108.6279 \\
\hline$A(5,1,7)$ & 111.0682 \\
\hline$A(5,1,15)$ & 107.1027 \\
\hline$A(6,1,7)$ & 111.2875 \\
\hline$A(6,1,15)$ & 108.0586 \\
\hline$A(7,1,15)$ & 110.5483 \\
\hline$A(2,14,3)$ & 105.9896 \\
\hline$A(2,14,15)$ & 106.4955 \\
\hline$A(2,14,17)$ & 113.2867 \\
\hline$A(3,14,15)$ & 104.1099 \\
\hline$A(3,14,17)$ & 110.5321 \\
\hline$A(15,14,17)$ & 115.6088 \\
\hline$A(1,15,4)$ & 108.2740 \\
\hline$A(1,15,14)$ & 110.7448 \\
\hline$A(1,15,16)$ & 114.2421 \\
\hline$A(4,15,14)$ & 103.1492 \\
\hline$A(4,15,16)$ & 109.8830 \\
\hline$A(14,15,16)$ & 109.9532 \\
\hline$A(8,16,9)$ & 113.7482 \\
\hline$A(8,16,10)$ & 113.2387 \\
\hline$A(8,16,15)$ & 104.0359 \\
\hline$A(9,16,10)$ & 114.0125 \\
\hline$A(9,16,15)$ & 106.0141 \\
\hline$A(10,16,15)$ & 104.4994 \\
\hline$A(11,17,12)$ & 113.4681 \\
\hline$A(11,17,13)$ & 113.3474 \\
\hline$A(11,17,14)$ & 104.0696 \\
\hline$A(12,17,13)$ & 113.8209 \\
\hline$A(12,17,14)$ & 103.6530 \\
\hline$A(13,17,14)$ & 107.2673 \\
\hline$D(5,1,15,4)$ & 61.2482 \\
\hline$D(5,1,15,14)$ & 173.6594 \\
\hline$D(5,1,15,16)$ & -61.5374 \\
\hline$D(6,1,15,4)$ & 178.1098 \\
\hline$D(6,1,15,14)$ & -69.4789 \\
\hline$D(6,1,15,16)$ & 55.3242 \\
\hline$D(7,1,15,4)$ & -59.8832 \\
\hline$D(7,1,15,14)$ & 52.5280 \\
\hline$D(7,1,15,16)$ & 177.3312 \\
\hline$D(2,14,15,1)$ & 51.0418 \\
\hline $\mathrm{D}(2,14,15,4)$ & 166.6879 \\
\hline $\mathrm{D}(2,14,15,16)$ & -76.1587 \\
\hline
\end{tabular}




$\begin{array}{lr}\text { D }(3,14,15,1) & 162.7982 \\ \text { D }(3,14,15,4) & -81.5556 \\ \text { D }(3,14,15,16) & 35.5977 \\ \text { D }(17,14,15,1) & -75.7675 \\ \text { D }(17,14,15,4) & 39.8787 \\ \text { D }(17,14,15,16) & 157.0320 \\ \text { D }(2,14,17,11) & 65.9485 \\ \text { D }(2,14,17,12) & -175.1458 \\ \text { D }(2,14,17,13) & -54.4324 \\ \text { D }(3,14,17,11) & -52.8302 \\ \text { D }(3,14,17,12) & 66.0754 \\ \text { D }(3,14,17,13) & -173.2111 \\ \text { D }(15,14,17,11) & -170.7471 \\ \text { D }(15,14,17,12) & -51.8414 \\ \text { D }(15,14,17,13) & 68.8720 \\ \text { D }(1,15,16,8) & 179.4845 \\ \text { D }(1,15,16,9) & 59.2446 \\ \text { D }(1,15,16,10) & -61.5204 \\ \text { D }(4,15,16,8) & 57.5777 \\ \text { D }(4,15,16,9) & -62.6623 \\ \text { D }(4,15,16,10) & 176.5728 \\ \text { D }(14,15,16,8) & -55.2932 \\ \text { D }(14,15,16,9) & -175.5332 \\ \text { D }(14,15,16,10) & 63.7019\end{array}$




\section{1,1-Dimethylhydrazine bisborane (3c)}

\section{Neutral, ground state, CAM-B3LYP/6-311G(d,p)}

Atomic coordinates and value of zero point energy:

$\begin{array}{lrrr}\text { C } & 0.48076 & 0.87625 & -1.21518 \\ \mathrm{C} & 0.48075 & 0.87625 & 1.21519 \\ \mathrm{H} & -0.70219 & -1.36889 & -0.80791 \\ \mathrm{H} & -0.70219 & -1.36891 & 0.80789 \\ \mathrm{H} & 1.44174 & 1.38357 & -1.22084 \\ \mathrm{H} & 0.41702 & 0.23437 & -2.09134 \\ \mathrm{H} & -0.34213 & 1.58686 & -1.20618 \\ \mathrm{H} & 1.50692 & -1.70063 & 1.00878 \\ \mathrm{H} & 2.69239 & -0.41286 & 0 . \\ \mathrm{H} & 1.50692 & -1.70062 & -1.00879 \\ \mathrm{H} & -2.9881 & -1.01435 & -0.00001 \\ \mathrm{H} & -2.35928 & 0.59912 & 1.01201 \\ \mathrm{H} & -2.35928 & 0.59914 & -1.012 \\ \mathrm{H} & 0.41701 & 0.23436 & 2.09135 \\ \mathrm{H} & 1.44173 & 1.38356 & 1.22085 \\ \mathrm{H} & -0.34213 & 1.58686 & 1.20619 \\ \mathrm{~N} & -0.79544 & -0.75156 & -0.00001 \\ \mathrm{~N} & 0.44748 & 0.02885 & 0 . \\ \mathrm{B} & 1.6755 & -1.05426 & 0 . \\ \mathrm{B} & -2.26784 & -0.04527 & 0 .\end{array}$

$\mathrm{ZPE}=-243.547999 \mathrm{Ha}$

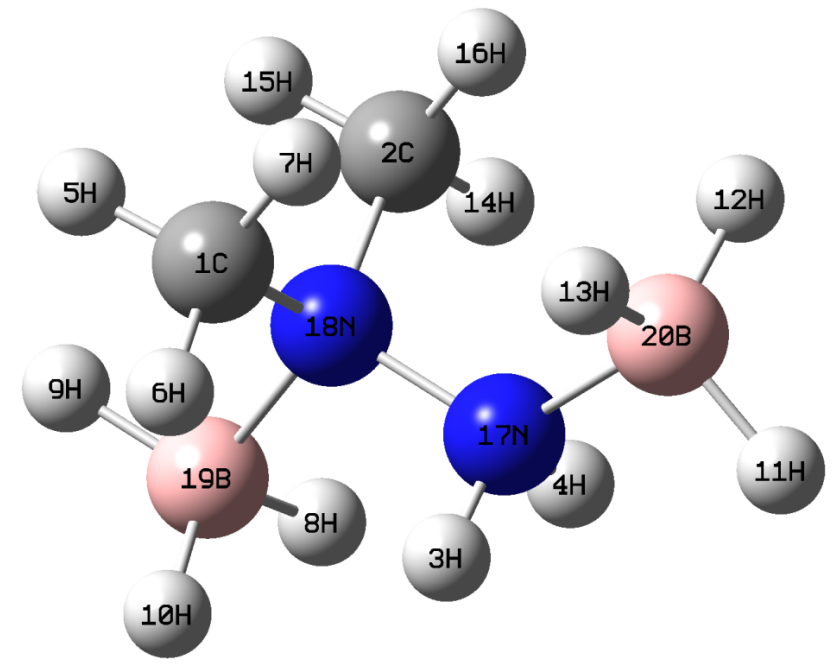

Geometrical parameters [bond distances (R) in $\AA$, angles (A) and dihedral (D) in degrees]:

$\begin{array}{ll}R(1,5) & 1.0867 \\ R(1,6) & 1.0880 \\ R(1,7) & 1.0873 \\ R(1,18) & 1.4818 \\ R(2,14) & 1.0880 \\ R(2,15) & 1.0867 \\ R(2,16) & 1.0873 \\ R(2,18) & 1.4818\end{array}$




\begin{tabular}{|c|c|}
\hline$R(3,17)$ & 1.0210 \\
\hline$R(4,17)$ & 1.0210 \\
\hline $\mathrm{R}(8,19)$ & 1.2099 \\
\hline$R(9,19)$ & 1.2023 \\
\hline$R(10,19)$ & 1.2099 \\
\hline$R(11,20)$ & 1.2074 \\
\hline$R(12,20)$ & 1.2032 \\
\hline$R(13,20)$ & 1.2032 \\
\hline $\mathrm{R}(17,18)$ & 1.4676 \\
\hline$R(17,20)$ & 1.6330 \\
\hline$R(18,19)$ & 1.6374 \\
\hline$A(5,1,6)$ & 108.8468 \\
\hline$A(5,1,7)$ & 111.3589 \\
\hline$A(5,1,18)$ & 106.9232 \\
\hline$A(6,1,7)$ & 110.3598 \\
\hline$A(6,1,18)$ & 108.7652 \\
\hline$A(7,1,18)$ & 110.4850 \\
\hline$A(14,2,15)$ & 108.8469 \\
\hline$A(14,2,16)$ & 110.3598 \\
\hline$A(14,2,18)$ & 108.7652 \\
\hline$A(15,2,16)$ & 111.3589 \\
\hline$A(15,2,18)$ & 106.9232 \\
\hline$A(16,2,18)$ & 110.4850 \\
\hline$A(3,17,4)$ & 104.6063 \\
\hline$A(3,17,18)$ & 104.1323 \\
\hline$A(3,17,20)$ & 110.1114 \\
\hline$A(4,17,18)$ & 104.1323 \\
\hline$A(4,17,20)$ & 110.1114 \\
\hline$A(18,17,20)$ & 122.2499 \\
\hline$A(1,18,2)$ & 110.1793 \\
\hline$A(1,18,17)$ & 108.8504 \\
\hline$A(1,18,19)$ & 111.1878 \\
\hline$A(2,18,17)$ & 108.8504 \\
\hline$A(2,18,19)$ & 111.1878 \\
\hline $\mathrm{A}(17,18,19)$ & 106.4640 \\
\hline$A(8,19,9)$ & 113.7569 \\
\hline$A(8,19,10)$ & 112.9769 \\
\hline$A(8,19,18)$ & 104.4115 \\
\hline$A(9,19,10)$ & 113.7569 \\
\hline$A(9,19,18)$ & 106.3464 \\
\hline $\mathrm{A}(10,19,18)$ & 104.4115 \\
\hline$A(11,20,12)$ & 112.6130 \\
\hline$A(11,20,13)$ & 112.6130 \\
\hline$A(11,20,17)$ & 100.9951 \\
\hline$A(12,20,13)$ & 114.5067 \\
\hline$A(12,20,17)$ & 107.4665 \\
\hline
\end{tabular}




\begin{tabular}{|c|c|}
\hline$A(13,20,17)$ & 107.4665 \\
\hline$D(5,1,18,2)$ & 64.7555 \\
\hline $\mathrm{D}(5,1,18,17)$ & -175.9557 \\
\hline$D(5,1,18,19)$ & -58.9855 \\
\hline$D(6,1,18,2)$ & -177.8579 \\
\hline$D(6,1,18,17)$ & -58.5691 \\
\hline $\mathrm{D}(6,1,18,19)$ & 58.4011 \\
\hline $\mathrm{D}(7,1,18,2)$ & -56.5809 \\
\hline$D(7,1,18,17)$ & 62.7079 \\
\hline$D(7,1,18,19)$ & 179.6781 \\
\hline $\mathrm{D}(14,2,18,1)$ & 177.8578 \\
\hline $\mathrm{D}(14,2,18,17)$ & 58.5690 \\
\hline$D(14,2,18,19)$ & -58.4012 \\
\hline$D(15,2,18,1)$ & -64.7556 \\
\hline$D(15,2,18,17)$ & 175.9556 \\
\hline $\mathrm{D}(15,2,18,19)$ & 58.9854 \\
\hline$D(16,2,18,1)$ & 56.5808 \\
\hline $\mathrm{D}(16,2,18,17)$ & -62.7080 \\
\hline$D(16,2,18,19)$ & -179.6782 \\
\hline$D(3,17,18,1)$ & 65.2609 \\
\hline$D(3,17,18,2)$ & -174.6247 \\
\hline $\mathrm{D}(3,17,18,19)$ & -54.6819 \\
\hline $\mathrm{D}(4,17,18,1)$ & 174.6242 \\
\hline $\mathrm{D}(4,17,18,2)$ & -65.2614 \\
\hline $\mathrm{D}(4,17,18,19)$ & 54.6814 \\
\hline $\mathrm{D}(20,17,18,1)$ & -60.0575 \\
\hline$D(20,17,18,2)$ & 60.0570 \\
\hline $\mathrm{D}(20,17,18,19)$ & -180.0003 \\
\hline $\mathrm{D}(3,17,20,11)$ & 57.4194 \\
\hline$D(3,17,20,12)$ & 175.5682 \\
\hline $\mathrm{D}(3,17,20,13)$ & -60.7295 \\
\hline$D(4,17,20,11)$ & -57.4197 \\
\hline$D(4,17,20,12)$ & 60.7291 \\
\hline $\mathrm{D}(4,17,20,13)$ & -175.5686 \\
\hline $\mathrm{D}(18,17,20,11)$ & -180.0002 \\
\hline $\mathrm{D}(18,17,20,12)$ & -61.8513 \\
\hline $\mathrm{D}(18,17,20,13)$ & 61.8510 \\
\hline$D(1,18,19,8)$ & -177.8313 \\
\hline $\mathrm{D}(1,18,19,9)$ & 61.5823 \\
\hline $\mathrm{D}(1,18,19,10)$ & -59.0040 \\
\hline $\mathrm{D}(2,18,19,8)$ & 59.0040 \\
\hline $\mathrm{D}(2,18,19,9)$ & -61.5824 \\
\hline$D(2,18,19,10)$ & 177.8313 \\
\hline $\mathrm{D}(17,18,19,8)$ & -59.4136 \\
\hline $\mathrm{D}(17,18,19,9)$ & -180.0000 \\
\hline $\mathrm{D}(17,18,19,10)$ & 59.4136 \\
\hline
\end{tabular}


Hydrazine borane dehydrogenation derivatives

\section{HYDRAZINE DEHYDROGENATED COMPOUNDS}

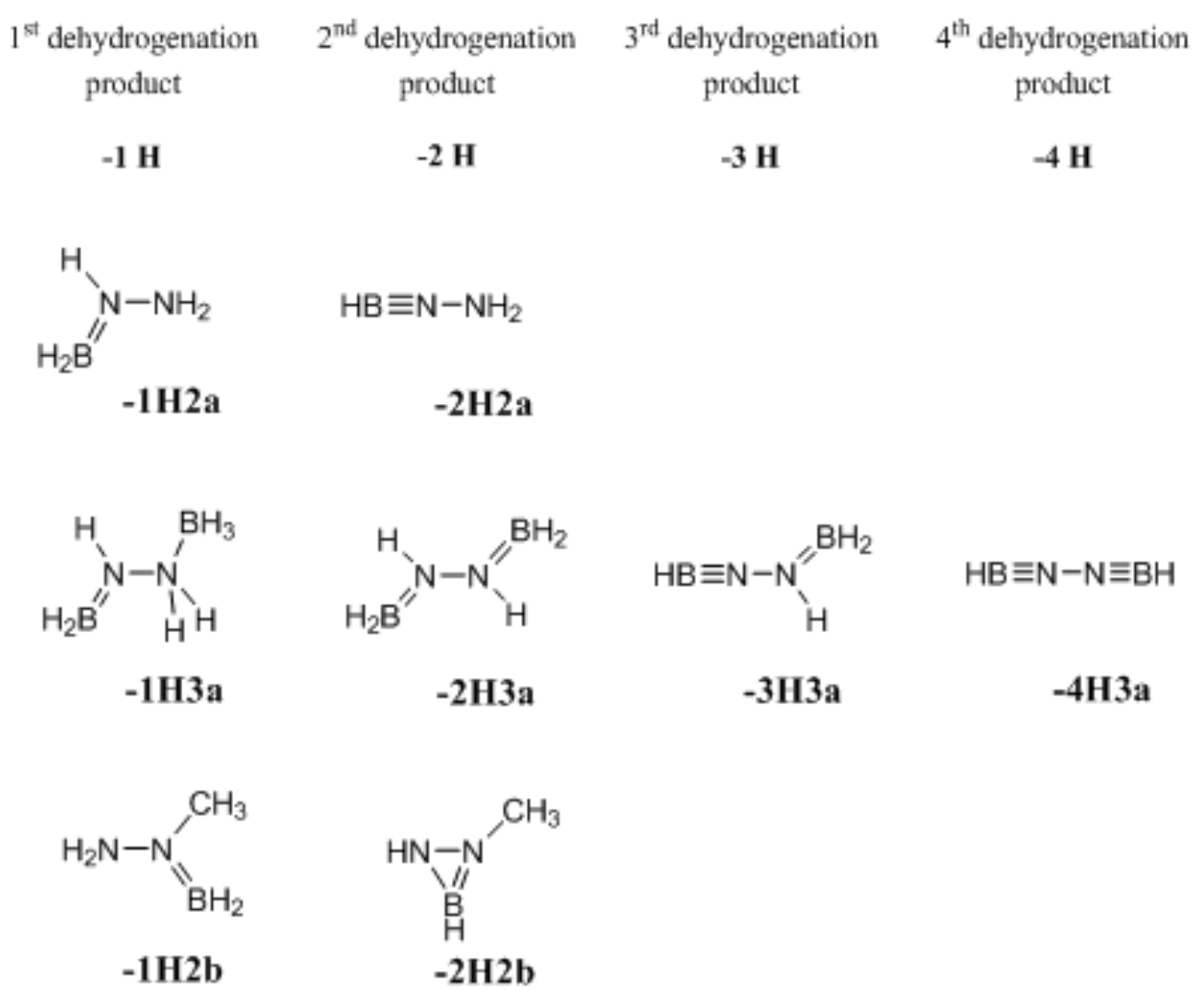<smiles></smiles><smiles>CN1BN1</smiles><smiles>BN(C)N=[BH2-]</smiles>

Chart 2 


\section{Hydrogen $\left(\mathrm{H}_{2}\right)$}

Neutral, ground state, CAM-B3LYP/6-311G (d,p)

Atomic coordinates and value of zero point energy:
$\mathrm{H} \quad 0.0000000000$
0.0000000000
0.3733457021
$\mathrm{H} \quad 0.0000000000$
0.0000000000
$-0.3733457021$

$\mathrm{ZPE}=-1.161093 \mathrm{Ha}$

\section{$1 \mathrm{H}-2 \mathrm{H}$}

Geometrical parameters bond distance (R) in $\AA$ :
$R(1,2)$
0.7467

Dehydrogenated hydrazine borane [-1H2a]

Neutral, ground state, CAM-B3LYP/6-311G $(d, p)$

Atomic coordinates and value of zero point energy: 


$\begin{array}{lrrr}\text { H } & 1.08615100 & -1.00103800 & 0.81722300 \\ \mathrm{H} & 1.08615100 & -1.00103800 & -0.81722300 \\ \mathrm{H} & 0.28520400 & 1.41935100 & 0.00000000 \\ \mathrm{H} & -2.15360800 & 0.93687300 & 0.00000000 \\ \mathrm{H} & -1.60265800 & -1.08279900 & 0.00000000 \\ \mathrm{~N} & 1.13426700 & -0.40376000 & 0.00000000 \\ \mathrm{~N} & 0.00000000 & 0.45211900 & 0.00000000 \\ \mathrm{~B} & -1.32822200 & 0.07802800 & 0.00000000\end{array}$

$\mathrm{ZPE}=-137.256285 \mathrm{Ha}$
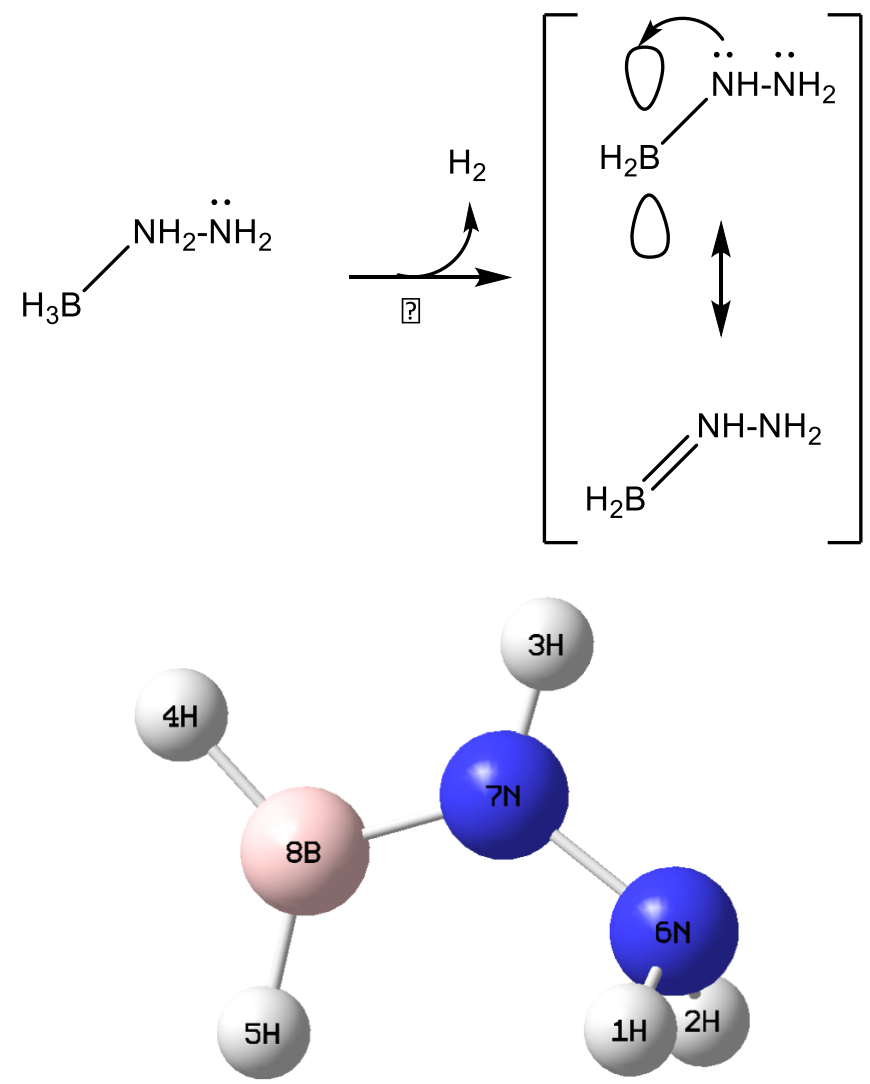

Geometrical parameters [bond distances (R) in $\AA$, angles (A) and dihedral (D) in degrees]:

$\begin{array}{ll}\mathrm{R}(1,6) & 1.0134 \\ \mathrm{R}(2,6) & 1.0134 \\ \mathrm{R}(3,7) & 1.0084 \\ \mathrm{R}(4,8) & 1.1912 \\ \mathrm{R}(5,8) & 1.1928 \\ \mathrm{R}(6,7) & 1.4209 \\ \mathrm{R}(7,8) & 1.3799 \\ \mathrm{~A}(1,6,2) & 107.50 \\ \mathrm{~A}(1,6,7) & 108.49 \\ \mathrm{~A}(2,6,7) & 108.49 \\ \mathrm{~A}(3,7,6) & 110.61 \\ \mathrm{~A}(3,7,8) & 122.16\end{array}$




$\begin{array}{lr}\text { A }(6,7,8) & 127.23 \\ \text { A }(4,8,5) & 122.84 \\ \text { A }(4,8,7) & 118.13 \\ \text { A }(5,8,7) & 119.03 \\ \text { D }(1,6,7,3) & 121.75 \\ \text { D }(1,6,7,8) & -58.25 \\ \text { D }(2,6,7,3) & -121.75 \\ \text { D }(2,6,7,8) & 58.25 \\ \text { D }(3,7,8,4) & 0.00 \\ \text { D }(3,7,8,5) & 180.00 \\ \text { D }(6,7,8,4) & 180.00 \\ \text { D }(6,7,8,5) & 0.00\end{array}$


Didehydrogenated hydrazine borane [-2H2a]

Neutral, ground state, CAM-B3LYP/6-311G (d,p)

Atomic coordinates and value of zero point energy:

$\begin{array}{crcr}\mathrm{H} & 0.39086 & -1.52223 & 0.82313 \\ \mathrm{H} & 0.39086 & -1.52223 & -0.82313 \\ \mathrm{H} & -0.05998 & 2.59402 & 0.00000 \\ \mathrm{~N} & -0.07509 & -1.1558 & 0.00000 \\ \mathrm{~N} & 0.00000 & 0.19984 & 0.00000 \\ \mathrm{~B} & -0.03922 & 1.42843 & 0.00000\end{array}$

$\mathrm{ZPE}=-136.041564 \mathrm{Ha}$

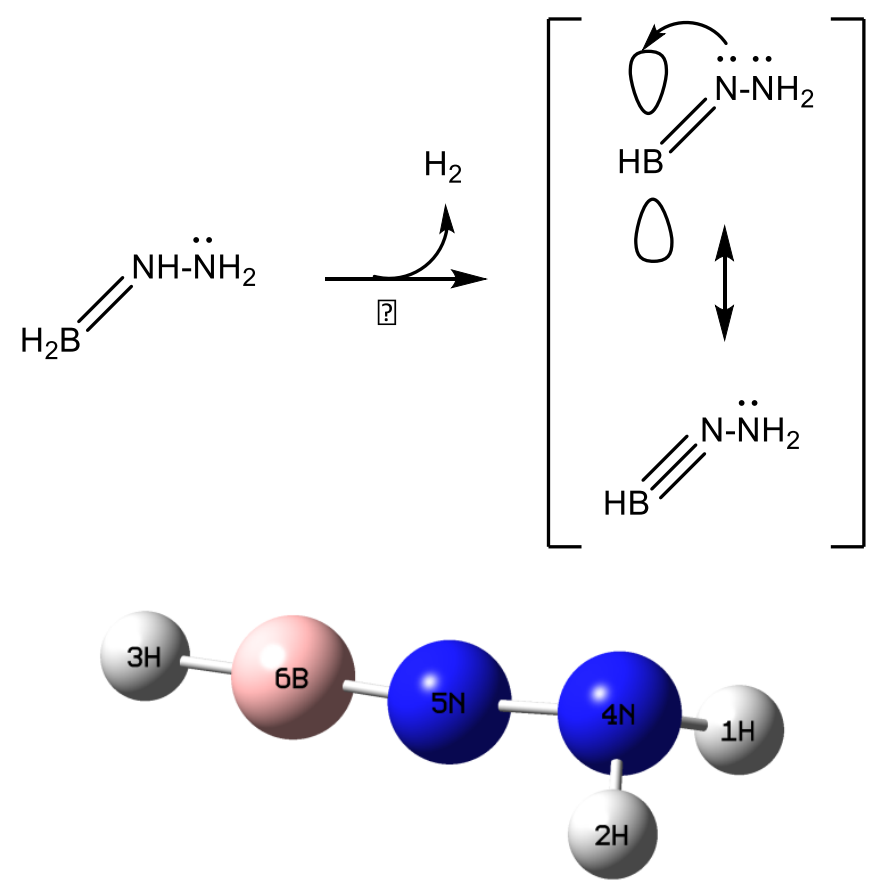

Geometrical parameters [bond distances (R) in $\AA$, angles (A) and dihedral (D) in degrees] :

$\begin{array}{lr}\mathrm{R}(1,4) & 1.0144 \\ \mathrm{R}(2,4) & 1.0144 \\ \mathrm{R}(3,6) & 1.1658 \\ \mathrm{R}(4,5) & 1.3577 \\ \mathrm{R}(5,6) & 1.2292 \\ \mathrm{~A}(1,4,2) & 108.4809 \\ \mathrm{~A}(1,4,5) & 109.5897 \\ \mathrm{~A}(2,4,5) & 109.5897 \\ \mathrm{~L}(4,5,6,1,-1) & 182.2267 \\ \mathrm{~L}(3,6,5,1,-1) & 179.6789 \\ \mathrm{~L}(4,5,6,1,-2) & 189.4677 \\ \mathrm{~L}(3,6,5,1,-2) & 182.5566 \\ \mathrm{D}(1,4,5,2) & 118.9331\end{array}$




\section{Dehydrogenated hydrazine bisborane [-1H3a]}

Neutral, ground state, CAM-B3LYP/6-311G $(d, p)$

Atomic coordinates and value of zero point energy:

$\begin{array}{lrrr}\mathrm{H} & 0.46327 & 1.05005 & -0.93978 \\ \mathrm{H} & 0.34483 & 1.18200 & 0.69347 \\ \mathrm{H} & 1.83199 & -0.79601 & 1.16388 \\ \mathrm{H} & 2.71464 & 0.61795 & 0.01672 \\ \mathrm{H} & 1.91233 & -1.02485 & -0.84340 \\ \mathrm{H} & -2.80900 & -0.82552 & 0.06683 \\ \mathrm{H} & -2.22316 & 1.18173 & 0.20000 \\ \mathrm{H} & -0.33063 & -1.31561 & -0.16245 \\ \mathrm{~N} & 0.45620 & 0.52014 & -0.07073 \\ \mathrm{~N} & -0.66043 & -0.36432 & -0.07265 \\ \mathrm{~B} & 1.88497 & -0.25476 & 0.08801 \\ \mathrm{~B} & -1.97991 & 0.02266 & 0.07367\end{array}$

$\mathrm{ZPE}=-163.866493 \mathrm{Ha}$
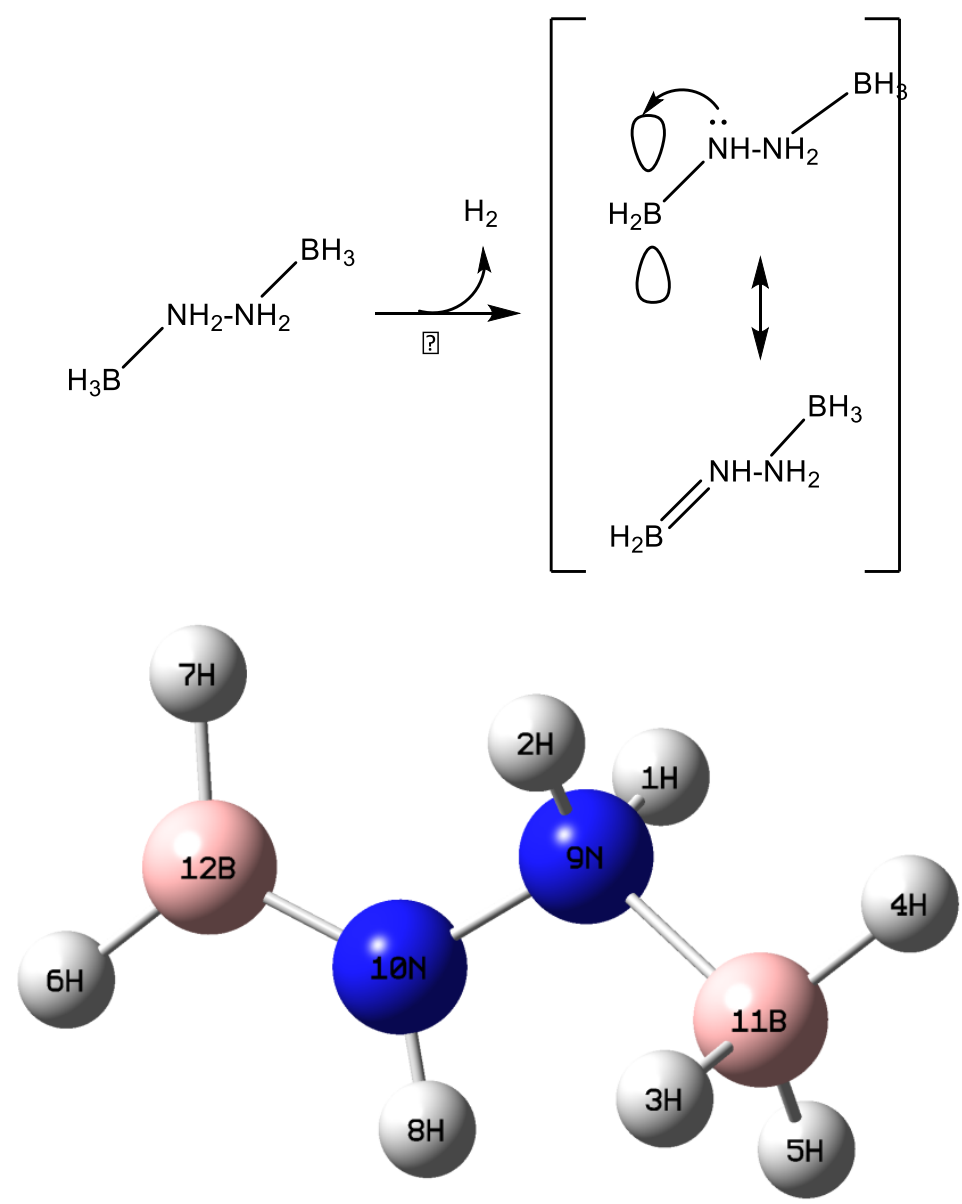

Geometrical parameters [bond distances (R) in $\AA$, angles (A) and dihedral (D) in degrees]:
$\mathrm{R}(1,9)$
1.0171
$\mathrm{R}(2,9)$
1.0179
$\mathrm{R}(3,11)$
1.2089
$\mathrm{R}(4,11)$
1.2063 


\begin{tabular}{|c|c|}
\hline $\mathrm{R}(5,11)$ & 1.2055 \\
\hline$R(6,12)$ & 1.1861 \\
\hline $\mathrm{R}(7,12)$ & 1.1910 \\
\hline$R(8,10)$ & 1.0108 \\
\hline$R(9,10)$ & 1.4245 \\
\hline$R(9,11)$ & 1.6331 \\
\hline$R(10,12)$ & 1.3828 \\
\hline$A(1,9,2)$ & 107.6672 \\
\hline$A(1,9,10)$ & 108.6162 \\
\hline$A(1,9,11)$ & 109.3617 \\
\hline$A(2,9,10)$ & 109.1192 \\
\hline$A(2,9,11)$ & 108.9008 \\
\hline$A(10,9,11)$ & 113.0366 \\
\hline$A(8,10,9)$ & 109.1897 \\
\hline$A(8,10,12)$ & 125.7385 \\
\hline$A(9,10,12)$ & 125.0347 \\
\hline $\mathrm{A}(3,11,4)$ & 113.5647 \\
\hline$A(3,11,5)$ & 113.7415 \\
\hline$A(3,11,9)$ & 104.3107 \\
\hline$A(4,11,5)$ & 114.0672 \\
\hline$A(4,11,9)$ & 104.6381 \\
\hline $\mathrm{A}(5,11,9)$ & 105.1493 \\
\hline $\mathrm{A}(6,12,7)$ & 123.6247 \\
\hline$A(6,12,10)$ & 117.7914 \\
\hline$A(7,12,10)$ & 118.5840 \\
\hline$D(1,9,10,8)$ & 132.9619 \\
\hline$D(1,9,10,12)$ & -44.9281 \\
\hline$D(2,9,10,8)$ & -109.9274 \\
\hline$D(2,9,10,12)$ & 72.1826 \\
\hline$D(11,9,10,8)$ & 114.0210 \\
\hline$D(11,9,10,12)$ & -166.4879 \\
\hline$D(1,9,11,3)$ & -178.9077 \\
\hline$D(1,9,11,4)$ & 61.5506 \\
\hline$D(1,9,11,5)$ & -58.9388 \\
\hline$D(2,9,11,3)$ & 63.6838 \\
\hline$D(2,9,11,4)$ & -55.8579 \\
\hline$D(2,9,11,5)$ & -176.3473 \\
\hline$D(10,9,11,3)$ & -57.7690 \\
\hline$D(10,9,11,4)$ & -177.3107 \\
\hline$D(10,9,11,5)$ & 62.1999 \\
\hline$D(8,10,12,6)$ & 167.2700 \\
\hline$D(8,10,12,7)$ & -178.2787 \\
\hline$D(9,10,12,6)$ & 179.2175 \\
\hline$D(9,10,12,7)$ & -0.7339 \\
\hline
\end{tabular}


Didehydrogenated hydrazine bisborane [-2H3a]

Neutral, ground state, CAM-B3LYP/6-311G $(d, p)$

Atomic coordinates and value of zero point energy:

\section{Conformer}

Total

Energy

( $\mathrm{Ha})$

$\Delta \mathrm{E}$

$(\mathrm{kcal} / \mathrm{mol})$
Form2

$-162.713471$

1.01
Form3

$-162.643276$

45.06
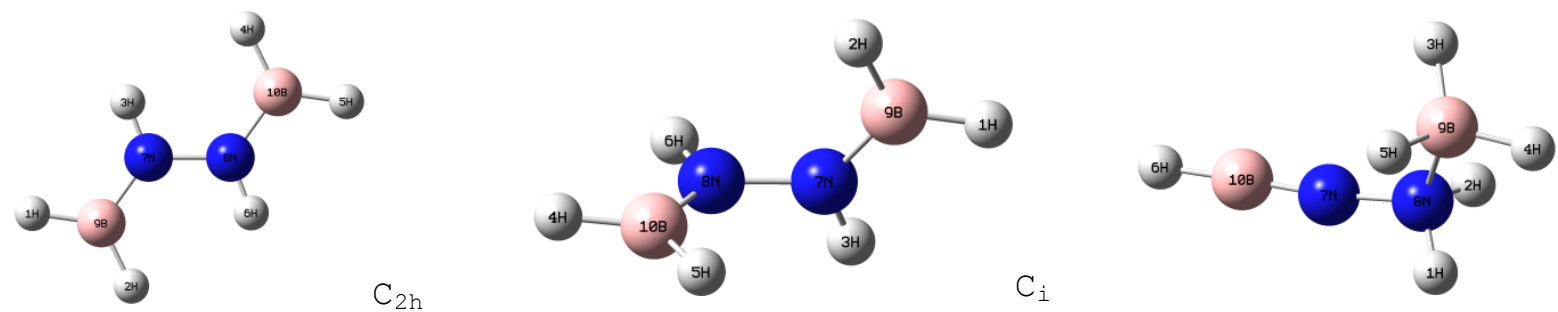

Form1: $\mathrm{ZPE}=-162.715082 \mathrm{Ha}$

$\mathrm{H}$
$\mathrm{H}$
$\mathrm{H}$
$\mathrm{H}$
$\mathrm{H}$
$\mathrm{H}$
$\mathrm{N}$
$\mathrm{N}$
$\mathrm{B}$
$\mathrm{B}$

$$
\begin{array}{r}
-0.36156 \\
1.50806 \\
-1.31987 \\
-1.50806 \\
0.36156 \\
1.31987 \\
-0.31635 \\
0.31635 \\
0.31635 \\
-0.31635
\end{array}
$$

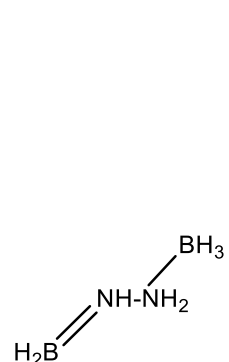

2.823730 .

1.874290

0.514550 .

-1.874290 .

-2.823730 .

-0.514550 .

0.623590 .

-0.623590 .

1.848610 .

$-1.848610$ 


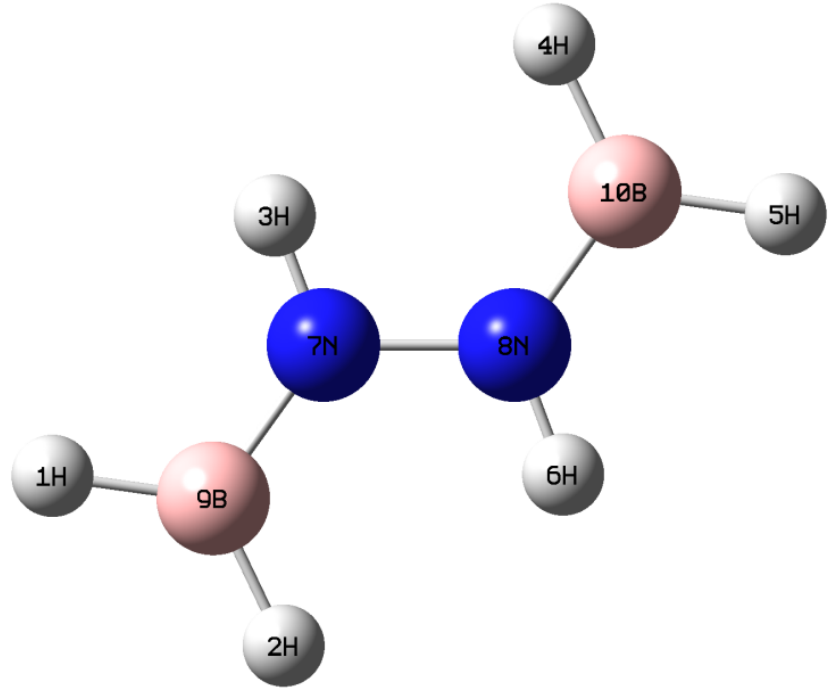

Geometrical parameters [bond distances (R) in $\AA$, angles (A) and dihedral (D) in degrees]:

\begin{tabular}{|c|c|}
\hline$R(1,9)$ & 1.1876 \\
\hline$R(2,9)$ & 1.1920 \\
\hline$R(3,7)$ & 1.0094 \\
\hline$R(4,10)$ & 1.1920 \\
\hline$R(5,10)$ & 1.1876 \\
\hline$R(6,8)$ & 1.0094 \\
\hline$R(7,8)$ & 1.3985 \\
\hline$R(7,9)$ & 1.3788 \\
\hline$R(8,10)$ & 1.3788 \\
\hline$A(3,7,8)$ & 110.6976 \\
\hline$A(3,7,9)$ & 123.5170 \\
\hline$A(8,7,9)$ & 125.7854 \\
\hline$A(6,8,7)$ & 110.6976 \\
\hline$A(6,8,10)$ & 123.5170 \\
\hline$A(7,8,10)$ & 125.7854 \\
\hline$A(1,9,2)$ & 123.5732 \\
\hline$A(1,9,7)$ & 117.8767 \\
\hline$A(2,9,7)$ & 118.5501 \\
\hline$A(4,10,5)$ & 123.5732 \\
\hline$A(4,10,8)$ & 118.5501 \\
\hline$A(5,10,8)$ & 117.8767 \\
\hline$D(3,7,8,6)$ & 180.0000 \\
\hline $\mathrm{D}(3,7,8,10)$ & 0.0000 \\
\hline$D(9,7,8,6)$ & 0.0000 \\
\hline $\mathrm{D}(9,7,8,10)$ & 180.0000 \\
\hline$D(3,7,9,1)$ & 0.0000 \\
\hline$D(3,7,9,2)$ & 180.0000 \\
\hline $\mathrm{D}(8,7,9,1)$ & 180.0000 \\
\hline$D(8,7,9,2)$ & 0.0000 \\
\hline $\mathrm{D}(6,8,10,4)$ & 180.0000 \\
\hline
\end{tabular}


$D(6,8,10,5) \quad 0.0000$

$D(7,8,10,4) \quad 0.0000$

$\mathrm{D}(7,8,10,5) \quad 180.0000$ 
Tridehydrogenated hydrazine bisborane [-3H3a]

Neutral, ground state, CAM-B3LYP/6-311G(d,p)

Atomic coordinates and value of zero point energy:

$\begin{array}{rrrr}\mathrm{H} & -1.36347 & -1.68693 & 0.00000 \\ \mathrm{H} & 0.46844 & -2.71171 & 0.00000 \\ \mathrm{H} & 1.54275 & -0.50183 & 0.00000 \\ \mathrm{H} & -0.97399 & 2.90265 & 0.00000 \\ \mathrm{~N} & 0.53228 & -0.51784 & 0.00000 \\ \mathrm{~N} & 0.00000 & 0.71490 & 0.00000 \\ \mathrm{~B} & -0.17764 & -1.71346 & 0.00000 \\ \mathrm{~B} & -0.50229 & 1.83714 & 0.00000\end{array}$

$\mathrm{ZPE}=-161.499980 \mathrm{Ha}$

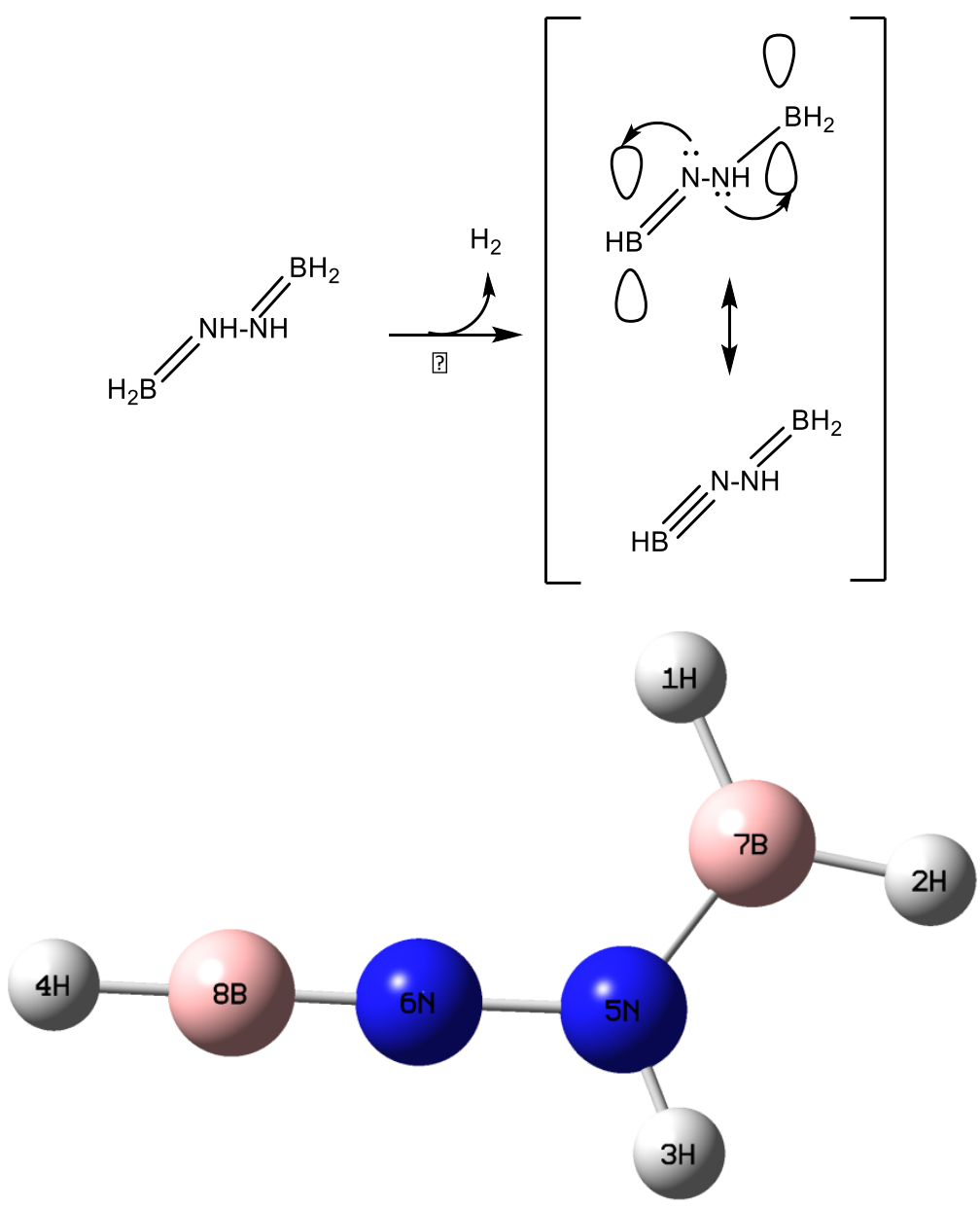

Geometrical parameters [bond distances (R) in $\AA$, angles (A) and dihedral (D) in degrees]:
$\mathrm{R}(1,7)$
1.1861
$R(2,7)$
1.1891
$R(3,5)$
1.0106
$\mathrm{R}(4,8)$
1.1653
$R(5,6)$
1. 3428
$R(5,7)$
1. 3905 


$\begin{array}{lr}\mathrm{R}(6,8) & 1.2295 \\ \mathrm{~A}(3,5,6) & 112.4458 \\ \mathrm{~A}(3,5,7) & 121.6085 \\ \mathrm{~A}(6,5,7) & 125.9456 \\ \mathrm{~A}(1,7,2) & 124.1932 \\ \mathrm{~A}(1,7,5) & 119.4189 \\ \mathrm{~A}(2,7,5) & 116.3879 \\ \mathrm{D}(3,5,7,1) & 180.0000 \\ \mathrm{D}(3,5,7,2) & 0.0000 \\ \mathrm{D}(6,5,7,1) & 0.0000 \\ \mathrm{D}(6,5,7,2) & 180.0000\end{array}$


Tetradehydrogenated hydrazine bisborane [ $-4 \mathrm{H} 3 \mathrm{a}]$

Neutral, ground state, CAM-B3LYP/6-311G $(d, p)$

Atomic coordinates and value of zero point energy:

$\begin{array}{lllr}H & 0 . & 0 . & 3.0383 \\ H & 0 . & 0 . & -3.0383 \\ N & 0 . & 0 . & -0.63987 \\ N & 0 . & 0 . & 0.63987 \\ B & 0 . & 0 . & 1.87372 \\ B & 0 . & 0 . & -1.87372\end{array}$

$\mathrm{ZPE}=-160.292698 \mathrm{Ha}$

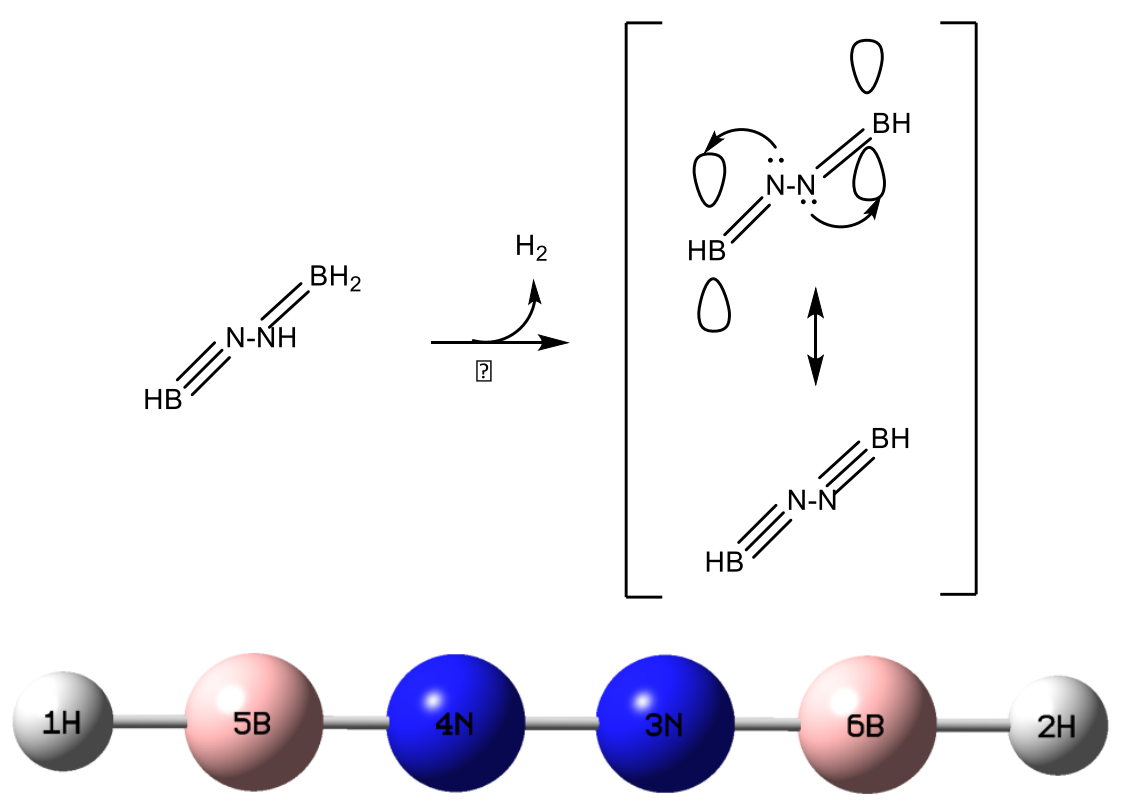

Geometrical parameters [bond distances

(R) in $\AA$, angles (A) and dihedral

(D) in degrees ] :

$\begin{array}{lr}R(1,5) & 1.1646 \\ R(2,6) & 1.1646 \\ R(3,4) & 1.2797 \\ R(3,6) & 1.2339 \\ R(4,5) & 1.2339 \\ A(4,3,6) & 180.0000 \\ A(3,4,5) & 180.0000 \\ A(1,5,4) & 180.0000 \\ A(2,6,3) & 180.0000\end{array}$




\section{Dehydrogenated methylhydrazine borane [-1H2b]}

Neutral, ground state, CAM-B3LYP/6-311G $(d, p)$

Atomic coordinates and value of zero point energy:

$\begin{array}{lrrr}\mathrm{C} & 1.37743 & -0.12248 & -0.01789 \\ \mathrm{H} & -0.92006 & -1.45698 & 0.87059 \\ \mathrm{H} & -1.68573 & -0.89367 & -0.45697 \\ \mathrm{H} & 1.90299 & 0.82965 & 0.01608 \\ \mathrm{H} & 1.68479 & -0.72593 & 0.84199 \\ \mathrm{H} & 1.64525 & -0.66622 & -0.92594 \\ \mathrm{H} & -1.85022 & 1.41369 & 0.02943 \\ \mathrm{H} & 0.02468 & 2.33502 & 0.01556 \\ \mathrm{~N} & -0.77254 & -1.10648 & -0.07308 \\ \mathrm{~N} & -0.05260 & 0.12051 & 0.01457 \\ \mathrm{~B} & -0.65807 & 1.36022 & 0.02524\end{array}$

$\mathrm{ZPE}=-176.521007 \mathrm{Ha}$
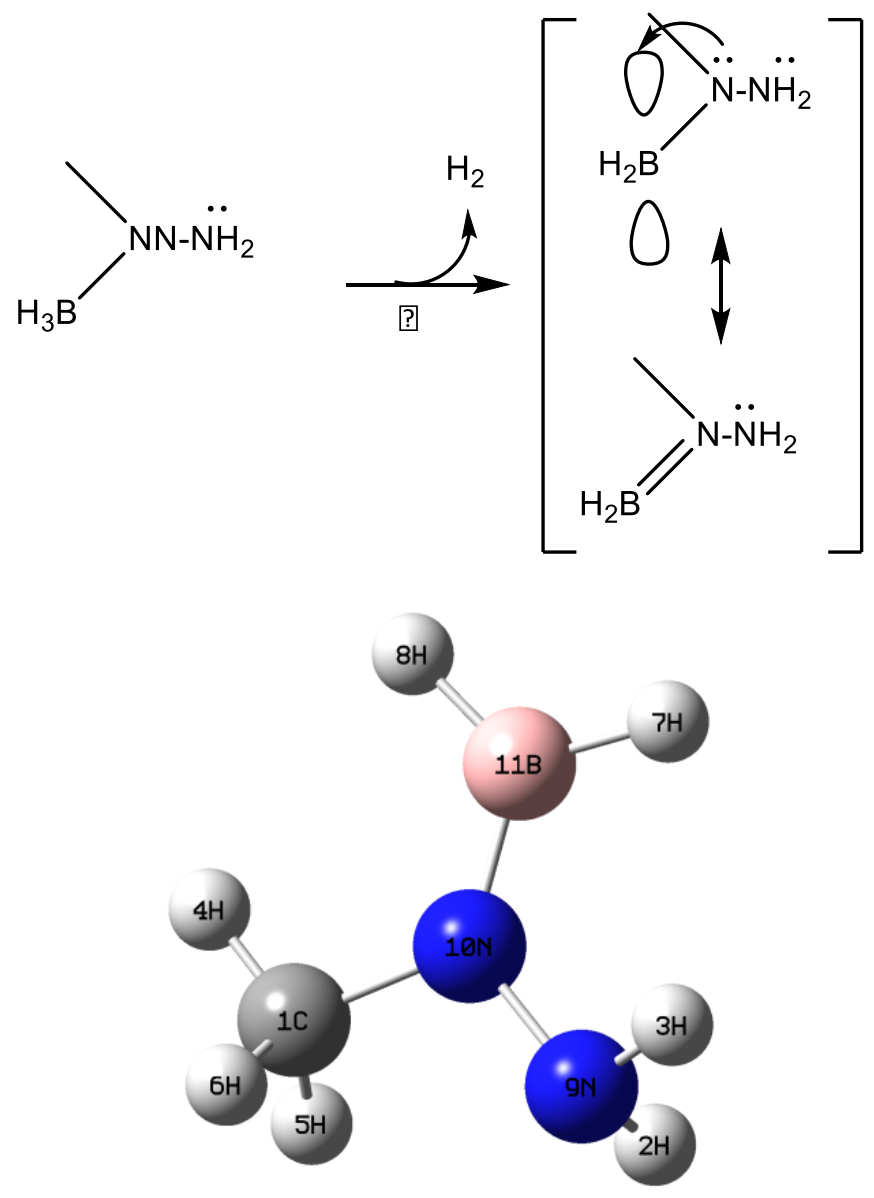

Geometrical parameters [bond distances (R) in $\AA$, angles (A) and dihedral (D) in degrees ] :
$R(1,4)$
1.0881
$R(1,5)$
1.0945
$R(1,6)$
1.0918 


$\begin{array}{lr}\text { R }(1,10) & 1.4509 \\ \text { R }(2,9) & 1.0174 \\ \text { R }(3,9) & 1.0132 \\ \text { R }(7,11) & 1.1933 \\ \text { R }(8,11) & 1.1902 \\ \text { R }(9,10) & 1.4253 \\ \text { R }(10,11) & 1.3797 \\ \text { A }(4,1,5) & 108.8014 \\ \text { A }(4,1,6) & 110.0777 \\ \text { A }(4,1,10) & 109.1970 \\ \text { A }(5,1,6) & 108.0555 \\ \text { A }(5,1,10) & 110.5811 \\ \text { A }(6,1,10) & 110.1086 \\ \text { A }(2,9,3) & 107.0435 \\ \text { A }(2,9,10) & 108.2259 \\ \text { A }(3,9,10) & 107.3217 \\ \text { A }(1,10,9) & 110.6277 \\ \text { A }(1,10,11) & 125.6753 \\ \text { A }(9,10,11) & 123.5266 \\ \text { A }(7,11,8) & 122.4398 \\ \text { A }(7,11,10) & 118.5996 \\ \text { A }(8,11,10) & 118.9556 \\ \text { D }(4,1,10,9) & 178.0892 \\ \text { D (4,1,10,11) } & 27.1640 \\ \text { D }(5,1,10,9) & -62.2232 \\ \text { D }(5,1,10,11) & 122.4041 \\ \text { D }(6,1,10,9) & 57.1199 \\ \text { D }(6,1,10,11) & -118.2529 \\ \text { D }(2,9,10,1) & 89.7280 \\ \text { D }(2,9,10,11) & -94.7808 \\ \text { D }(3,9,10,1) & -155.0621 \\ \text { D }(3,9,10,11) & 20.4291 \\ \text { D }(1,10,11,7) & 178.7281 \\ \text { D }(1,10,11,8) & -0.4823 \\ \text { D }(9,10,11,7) & 39.2440 \\ \text { D }(9,10,11,8) & -175.2860\end{array}$




\section{Didehydrogenated methylhydrazine borane [-2H2b] Neutral, ground state, CAM-B3LYP/6-311G $(d, p)$}
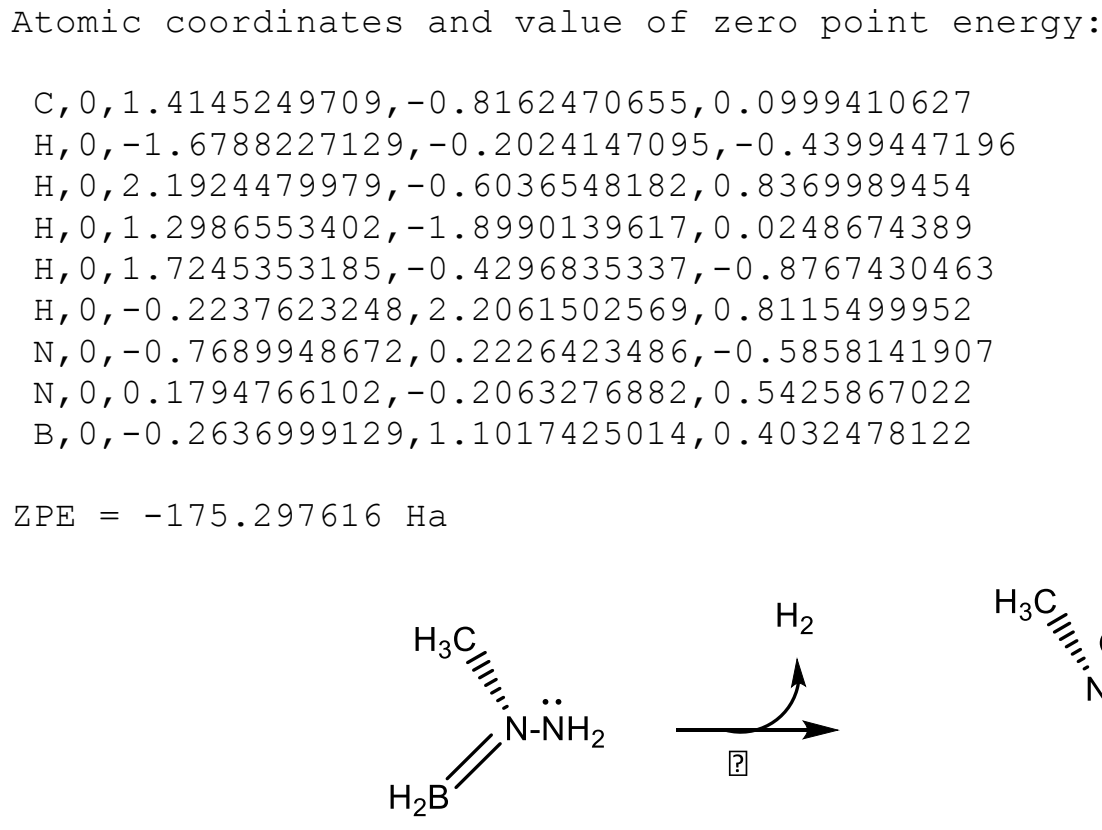<smiles>C[C@@H]1[B-]N1</smiles>

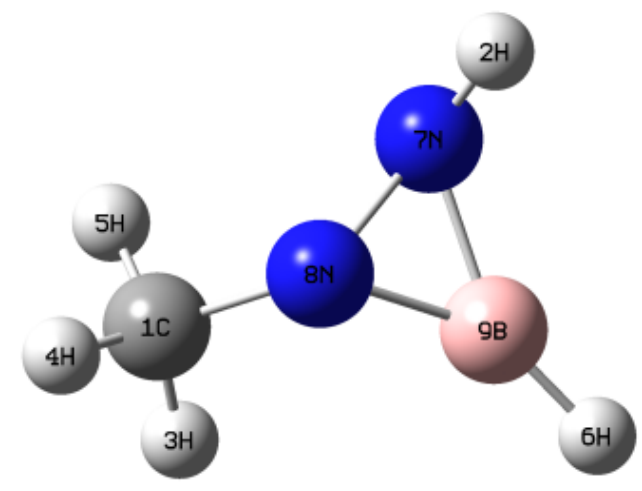

Geometrical parameters [bond distances (R) in $\AA$, angles (A) and dihedral (D) in degrees ]:
$R(1,3)$
1.0925
$R(1,4)$
1.0915
$\mathrm{R}(1,5)$
1.0952
$\mathrm{R}(1,8)$
1. 4468
$R(2,7)$
1.0148
$\mathrm{R}(6,9)$
1.1781
$\mathrm{R}(7,8)$
1.5352
$R(7,9)$
1. 4165
$\mathrm{R}(8,9)$
1.3881
$A(3,1,4)$
108.3614
$A(3,1,5)$
109.3537
$A(3,1,8)$
108.6259
$A(4,1,5)$
108.5929
$A(4,1,8)$
110.4017 


$\begin{array}{lr}\text { A }(5,1,8) & 111.4492 \\ A(2,7,8) & 109.3432 \\ \text { A }(2,7,9) & 118.6484 \\ \text { A }(1,8,7) & 114.8535 \\ \text { A }(1,8,9) & 129.7228 \\ \text { A }(6,9,7) & 146.7065 \\ \text { A }(6,9,8) & 146.9029 \\ \text { D }(3,1,8,7) & 150.3345 \\ \text { D }(3,1,8,9) & 82.7144 \\ \text { D }(4,1,8,7) & -90.9785 \\ \text { D }(4,1,8,9) & -158.5985 \\ \text { D }(5,1,8,7) & 29.7933 \\ \text { D }(5,1,8,9) & -37.8267 \\ \text { D }(2,7,8,1) & 125.2019 \\ \text { D }(2,7,8,9) & -112.0861 \\ \text { D }(2,7,9,6) & -86.7264 \\ \text { D }(8,7,9,6) & 178.3299 \\ \text { D }(1,8,9,6) & -81.3574\end{array}$


Dehydrogenated methylhydrazine bisborane [-1H3b]

Neutral, ground state, CAM-B3LYP/6-311G(d,p)

Atomic coordinates and value of zero point energy:

$\begin{array}{rrrr}\mathrm{C} & -0.73948 & 1.36221 & 0.04094 \\ \mathrm{H} & 0.84216 & -0.33584 & 1.42958 \\ \mathrm{H} & 0.68917 & -1.54048 & 0.33101 \\ \mathrm{H} & -1.77588 & 1.59956 & -0.18397 \\ \mathrm{H} & -0.47564 & 1.79678 & 1.00947 \\ \mathrm{H} & -0.08434 & 1.78562 & -0.71855 \\ \mathrm{H} & -2.66181 & -0.61381 & -0.58411 \\ \mathrm{H} & -1.31584 & -2.16700 & -0.19705 \\ \mathrm{H} & 1.72430 & -0.14153 & -1.56209 \\ \mathrm{H} & 2.88512 & -0.64142 & 0.01683 \\ \mathrm{H} & 2.11017 & 1.20661 & -0.10065 \\ \mathrm{~N} & -0.61876 & -0.08679 & 0.07804 \\ \mathrm{~N} & 0.69732 & -0.52871 & 0.43726 \\ \mathrm{~B} & -1.59250 & -1.00949 & -0.25835 \\ \mathrm{~B} & 1.98242 & 0.04683 & -0.40030\end{array}$

$\mathrm{ZPE}=-203.127804 \mathrm{Ha}$
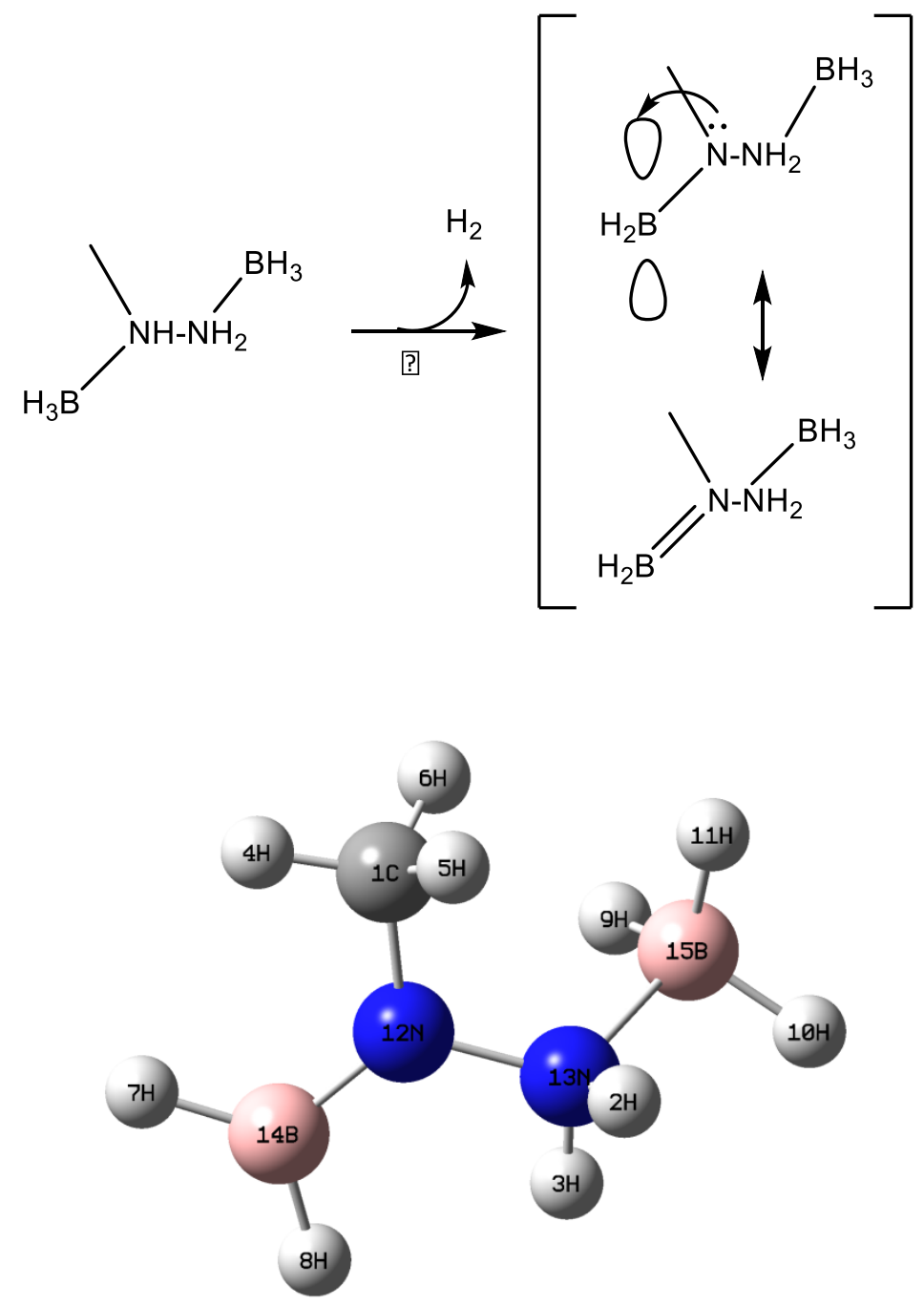
Geometrical parameters [bond distances (R) in $\AA$, angles $(A)$ and dihedral (D) in degrees] :

\begin{tabular}{|c|c|}
\hline$R(1,4)$ & 1.0868 \\
\hline$R(1,5)$ & 1.0939 \\
\hline$R(1,6)$ & 1.0887 \\
\hline$R(1,12)$ & 1.4545 \\
\hline$R(2,13)$ & 1.0212 \\
\hline$R(3,13)$ & 1.0174 \\
\hline$R(7,14)$ & 1.1858 \\
\hline$R(8,14)$ & 1.1917 \\
\hline$R(9,15)$ & 1.2049 \\
\hline$R(10,15)$ & 1.2094 \\
\hline$R(11,15)$ & 1.2047 \\
\hline$R(12,13)$ & 1.4340 \\
\hline$R(12,14)$ & 1.3830 \\
\hline$R(13,15)$ & 1.6384 \\
\hline$A(4,1,5)$ & 109.0568 \\
\hline$A(4,1,6)$ & 110.1552 \\
\hline$A(4,1,12)$ & 107.5785 \\
\hline$A(5,1,6)$ & 108.5435 \\
\hline$A(5,1,12)$ & 110.6813 \\
\hline$A(6,1,12)$ & 110.8110 \\
\hline$A(1,12,13)$ & 112.9277 \\
\hline$A(1,12,14)$ & 126.8704 \\
\hline$A(13,12,14)$ & 120.0993 \\
\hline$A(2,13,3)$ & 106.8841 \\
\hline$A(2,13,12)$ & 108.3843 \\
\hline$A(2,13,15)$ & 108.6117 \\
\hline$A(3,13,12)$ & 105.8407 \\
\hline$A(3,13,15)$ & 107.5925 \\
\hline$A(12,13,15)$ & 118.9179 \\
\hline$A(7,14,8)$ & 123.2022 \\
\hline$A(7,14,12)$ & 118.6272 \\
\hline$A(8,14,12)$ & 118.1685 \\
\hline$A(9,15,10)$ & 113.7970 \\
\hline$A(9,15,11)$ & 114.3967 \\
\hline$A(9,15,13)$ & 105.6625 \\
\hline$A(10,15,11)$ & 112.5164 \\
\hline$A(10,15,13)$ & 102.0777 \\
\hline$A(11,15,13)$ & 107.1113 \\
\hline$D(4,1,12,13)$ & -176.9220 \\
\hline$D(4,1,12,14)$ & 67.9320 \\
\hline$D(5,1,12,13)$ & -57.8648 \\
\hline$D(5,1,12,14)$ & 125.8504 \\
\hline$D(6,1,12,13)$ & 62.6084 \\
\hline $\mathrm{D}(6,1,12,14)$ & -113.6764 \\
\hline
\end{tabular}




$\begin{array}{lr}\text { D }(1,12,13,2) & 71.6985 \\ \text { D }(1,12,13,3) & -173.9433 \\ \text { D }(1,12,13,15) & -52.9005 \\ \text { D }(14,12,13,2) & -111.7365 \\ \text { D }(14,12,13,3) & 26.2170 \\ \text { D }(14,12,13,15) & 123.6645 \\ \text { D }(1,12,14,7) & -20.5720 \\ \text { D }(1,12,14,8) & 178.4471 \\ \text { D }(13,12,14,7) & -178.1019 \\ \text { D }(13,12,14,8) & 24.0250 \\ \text { D }(2,13,15,9) & -176.3489 \\ \text { D }(2,13,15,10) & 64.4066 \\ \text { D }(2,13,15,11) & -53.9936 \\ \text { D }(3,13,15,9) & 68.2927 \\ \text { D }(3,13,15,10) & -50.9519 \\ \text { D }(3,13,15,11) & -169.3521 \\ \text { D }(12,13,15,9) & -51.8604 \\ \text { D }(12,13,15,10) & -171.1049 \\ \text { D }(12,13,15,11) & 70.4949\end{array}$


Didehydrogenated methylhydrazine bisborane [-2H3b] Neutral, ground state, CAM-B3LYP/6-311G(d,p)

Atomic coordinates and value of zero point energy:

$\begin{array}{lrrr}\text { C } & -1.27056 & 1.03084 & -0.15991 \\ \mathrm{H} & 0.94976 & 0.52170 & 1.39135 \\ \mathrm{H} & -2.28407 & 0.71267 & -0.39377 \\ \mathrm{H} & -1.29823 & 1.68370 & 0.71812 \\ \mathrm{H} & -0.86388 & 1.60099 & -0.99889 \\ \mathrm{H} & -0.10721 & -2.33408 & 0.23861 \\ \mathrm{H} & -2.00030 & -1.65848 & -0.33478 \\ \mathrm{H} & 1.81184 & -0.33826 & -1.50210 \\ \mathrm{H} & 3.02865 & 0.35887 & 0.05468 \\ \mathrm{~N} & 0.87005 & 0.18536 & 0.44093 \\ \mathrm{~N} & -0.45344 & -0.14370 & 0.09876 \\ \mathrm{~B} & -0.87351 & -1.46091 & -0.00620 \\ \mathrm{~B} & 1.96761 & 0.05615 & -0.39212\end{array}$
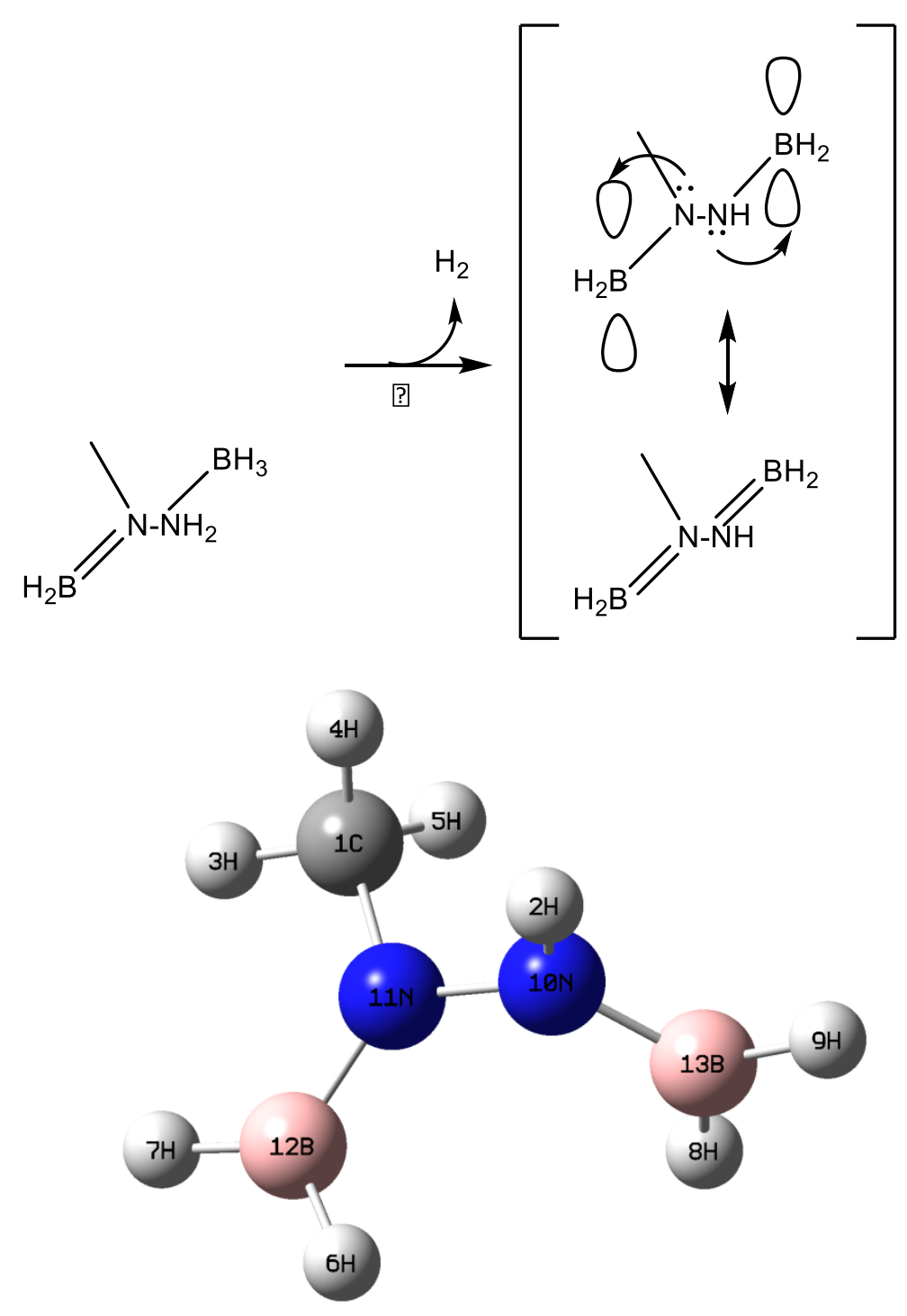
Geometrical parameters [bond distances (R) in $\AA$, angles $(A)$ and dihedral (D) in degrees] :

\begin{tabular}{|c|c|}
\hline$R(1,3)$ & 1.0877 \\
\hline$R(1,4)$ & 1.0945 \\
\hline$R(1,5)$ & 1.0929 \\
\hline $\mathrm{R}(1,11)$ & 1.4540 \\
\hline$R(2,10)$ & 1.0113 \\
\hline $\mathrm{R}(6,12)$ & 1.1873 \\
\hline $\mathrm{R}(7,12)$ & 1.1902 \\
\hline$R(8,13)$ & 1.1882 \\
\hline$R(9,13)$ & 1.1904 \\
\hline$R(10,11)$ & 1.4060 \\
\hline$R(10,13)$ & 1.3839 \\
\hline$R(11,12)$ & 1.3866 \\
\hline$A(3,1,4)$ & 108.8691 \\
\hline$A(3,1,5)$ & 109.5294 \\
\hline$A(3,1,11)$ & 109.0018 \\
\hline$A(4,1,5)$ & 108.3048 \\
\hline$A(4,1,11)$ & 110.6912 \\
\hline $\mathrm{A}(5,1,11)$ & 110.4185 \\
\hline$A(2,10,11)$ & 112.3780 \\
\hline$A(2,10,13)$ & 122.2916 \\
\hline$A(11,10,13)$ & 125.3220 \\
\hline$A(1,11,10)$ & 112.5341 \\
\hline$A(1,11,12)$ & 125.7090 \\
\hline$A(10,11,12)$ & 121.7299 \\
\hline$A(6,12,7)$ & 123.0840 \\
\hline$A(6,12,11)$ & 119.1783 \\
\hline$A(7,12,11)$ & 117.7366 \\
\hline$A(8,13,9)$ & 123.4959 \\
\hline$A(8,13,10)$ & 119.2955 \\
\hline$A(9,13,10)$ & 117.2076 \\
\hline$D(3,1,11,10)$ & -178.6073 \\
\hline$D(3,1,11,12)$ & 32.6510 \\
\hline $\mathrm{D}(4,1,11,10)$ & -58.8918 \\
\hline$D(4,1,11,12)$ & 122.9805 \\
\hline$D(5,1,11,10)$ & 61.0312 \\
\hline$D(5,1,11,12)$ & -117.0964 \\
\hline$D(2,10,11,1)$ & 76.2454 \\
\hline $\mathrm{D}(2,10,11,12)$ & -105.5421 \\
\hline $\mathrm{D}(13,10,11,1)$ & -104.7949 \\
\hline $\mathrm{D}(13,10,11,12)$ & 73.4176 \\
\hline$D(2,10,13,8)$ & -179.6220 \\
\hline$D(2,10,13,9)$ & 0.0303 \\
\hline$D(11,10,13,8)$ & 151.5900 \\
\hline$D(11,10,13,9)$ & -178.8319 \\
\hline
\end{tabular}




$\begin{array}{lr}D(1,11,12,6) & -179.7056 \\ D(1,11,12,7) & -0.0841 \\ D(10,11,12,6) & 23.2780 \\ D(10,11,12,7) & -178.0507\end{array}$


Tridehydrogenated methylhydrazine bisborane [-3H3b]

Neutral, ground state, CAM-B3LYP/6-311G(d,p)

Atomic coordinates and value of zero point energy:

$\begin{array}{lrrr}\mathrm{C} & 1.44893 & 0.60045 & 0 . \\ \mathrm{H} & 1.67013 & 1.66477 & 0 . \\ \mathrm{H} & 1.88238 & 0.13484 & 0.88776 \\ \mathrm{H} & 1.88238 & 0.13484 & -0.88776 \\ \mathrm{H} & -2.08903 & 1.19132 & 0 . \\ \mathrm{H} & -0.5152 & 2.57205 & 0 . \\ \mathrm{H} & -0.95655 & -3.19044 & 0 . \\ \mathrm{N} & -0.36666 & -0.86865 & 0 . \\ \mathrm{N} & 0 . & 0.42614 & 0 . \\ \mathrm{B} & -0.93368 & 1.45932 & 0 . \\ \mathrm{B} & -0.66654 & -2.06181 & 0 .\end{array}$

$\mathrm{ZPE}=-200.765242 \mathrm{Ha}$
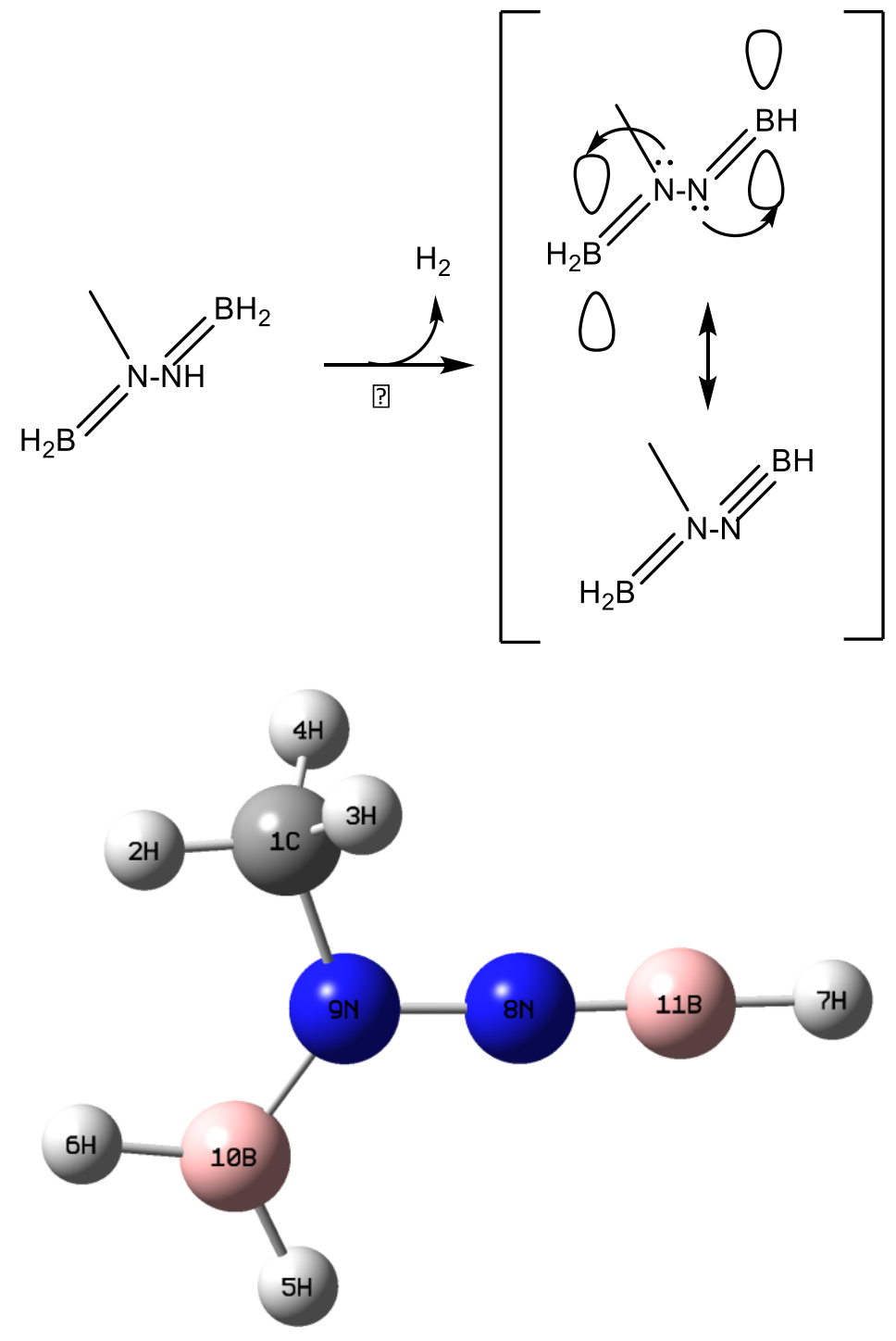
Geometrical parameters [bond distances (R) in $\AA$, angles $(A)$ and dihedral (D) in degrees] :

\begin{tabular}{|c|c|}
\hline$R(1,2)$ & 1.0871 \\
\hline$R(1,3)$ & 1.0921 \\
\hline$R(1,4)$ & 1.0921 \\
\hline$R(1,9)$ & 1.4594 \\
\hline$R(5,10)$ & 1.1860 \\
\hline$R(6,10)$ & 1.1888 \\
\hline $\mathrm{R}(7,11)$ & 1.1653 \\
\hline$R(8,9)$ & 1.3457 \\
\hline $\mathrm{R}(8,11)$ & 1.2303 \\
\hline$R(9,10)$ & 1.3926 \\
\hline$A(2,1,3)$ & 109.6729 \\
\hline$A(2,1,4)$ & 109.6729 \\
\hline$A(2,1,9)$ & 108.6003 \\
\hline$A(3,1,4)$ & 108.7524 \\
\hline$A(3,1,9)$ & 110.0665 \\
\hline$A(4,1,9)$ & 110.0665 \\
\hline$A(1,9,8)$ & 112.6710 \\
\hline$A(1,9,10)$ & 125.2439 \\
\hline$A(8,9,10)$ & 122.0850 \\
\hline$A(5,10,6)$ & 123.6695 \\
\hline$A(5,10,9)$ & 119.0446 \\
\hline$A(6,10,9)$ & 117.2859 \\
\hline $\mathrm{D}(9,8,11,5)$ & 180.0000 \\
\hline $\mathrm{D}(7,11,8,5)$ & 180.0000 \\
\hline $\mathrm{D}(2,1,9,8)$ & 180.0000 \\
\hline $\mathrm{D}(2,1,9,10)$ & 0.0000 \\
\hline$D(3,1,9,8)$ & -59.9276 \\
\hline $\mathrm{D}(3,1,9,10)$ & 120.0724 \\
\hline $\mathrm{D}(4,1,9,8)$ & 59.9276 \\
\hline $\mathrm{D}(4,1,9,10)$ & -120.0724 \\
\hline$D(1,9,10,5)$ & 180.0000 \\
\hline$D(1,9,10,6)$ & 0.0000 \\
\hline $\mathrm{D}(8,9,10,5)$ & 0.0000 \\
\hline$D(8,9,10,6)$ & 180.0000 \\
\hline
\end{tabular}


Dehydrogenated 1,1-Dimethylhydrazine borane [-1H2c]

Neutral, ground state, CAM-B3LYP/6-311G (d,p)

Atomic coordinates and value of zero point energy:

$\begin{array}{lrrr}\mathrm{C} & -0.82219 & 1.27136 & 0.08918 \\ \mathrm{C} & -0.9981 & -1.16827 & 0.01797 \\ \mathrm{H} & 1.12692 & 0.78616 & -1.26655 \\ \mathrm{H} & -1.51076 & 1.30672 & 0.93442 \\ \mathrm{H} & -0.09825 & 2.07643 & 0.19415 \\ \mathrm{H} & -1.38399 & 1.39099 & -0.84234 \\ \mathrm{H} & 1.74581 & 0.89166 & 1.16186 \\ \mathrm{H} & 1.61796 & -1.17035 & 1.15239 \\ \mathrm{H} & -0.38087 & -2.06079 & -0.0168 \\ \mathrm{H} & -1.63347 & -1.19569 & 0.90478 \\ \mathrm{H} & -1.61284 & -1.11586 & -0.88414 \\ \mathrm{~N} & 1.01145 & -0.12794 & -0.83736 \\ \mathrm{~N} & -0.11781 & -0.00553 & 0.09223 \\ \mathrm{~B} & 1.35916 & -0.11871 & 0.64705\end{array}$

$\mathrm{ZPE}=-215.765332 \mathrm{Ha}$
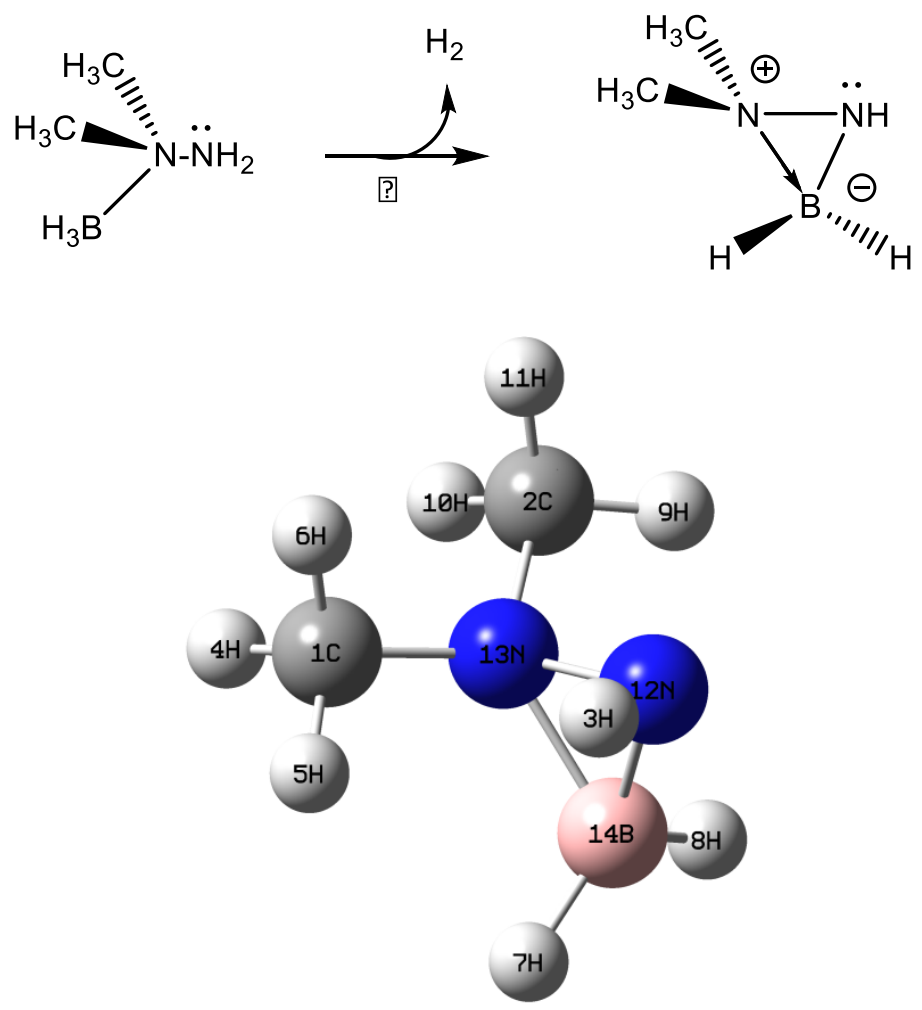
Geometrical parameters [bond distances (R) in $\AA$, angles (A) and dihedral
(D) in degrees]:
$R(1,4)$
1.0908
$R(1,5)$
1.0878
$R(1,6)$
1.0944
$R(1,13)$
1.4583
$R(2,9)$
1.0857
$\mathrm{R}(2,10)$
1.0913 


\begin{tabular}{|c|c|}
\hline$R(2,11)$ & 1.0929 \\
\hline$R(2,13)$ & 1.4603 \\
\hline$R(3,12)$ & 1.0164 \\
\hline$R(7,14)$ & 1.1981 \\
\hline $\mathrm{R}(8,14)$ & 1.1951 \\
\hline $\mathrm{R}(12,13)$ & 1.4678 \\
\hline$R(12,14)$ & 1.5246 \\
\hline$R(13,14)$ & 1.5818 \\
\hline$A(4,1,5)$ & 108.7444 \\
\hline$A(4,1,6)$ & 109.3894 \\
\hline$A(4,1,13)$ & 109.3705 \\
\hline$A(5,1,6)$ & 110.0528 \\
\hline$A(5,1,13)$ & 109.0493 \\
\hline$A(6,1,13)$ & 110.2091 \\
\hline$A(9,2,10)$ & 109.6556 \\
\hline$A(9,2,11)$ & 109.4363 \\
\hline$A(9,2,13)$ & 108.2697 \\
\hline$A(10,2,11)$ & 110.1506 \\
\hline$A(10,2,13)$ & 109.2482 \\
\hline$A(11,2,13)$ & 110.0511 \\
\hline$A(3,12,13)$ & 106.2499 \\
\hline$A(3,12,14)$ & 112.3194 \\
\hline$A(1,13,2)$ & 113.9490 \\
\hline$A(1,13,12)$ & 116.3161 \\
\hline$A(1,13,14)$ & 120.9571 \\
\hline$A(2,13,12)$ & 111.4191 \\
\hline$A(2,13,14)$ & 121.5830 \\
\hline$A(7,14,8)$ & 119.3736 \\
\hline$A(7,14,12)$ & 119.8067 \\
\hline$A(7,14,13)$ & 113.0612 \\
\hline$A(8,14,12)$ & 117.1135 \\
\hline$A(8,14,13)$ & 114.4236 \\
\hline$D(4,1,13,2)$ & 58.5412 \\
\hline$D(4,1,13,12)$ & -169.7408 \\
\hline$D(4,1,13,14)$ & -100.6370 \\
\hline$D(5,1,13,2)$ & 177.3415 \\
\hline$D(5,1,13,12)$ & -50.9405 \\
\hline$D(5,1,13,14)$ & 181.6330 \\
\hline$D(6,1,13,2)$ & -61.7511 \\
\hline$D(6,1,13,12)$ & 69.9669 \\
\hline$D(6,1,13,14)$ & 139.0707 \\
\hline$D(9,2,13,1)$ & 177.6014 \\
\hline$D(9,2,13,12)$ & 43.5494 \\
\hline$D(9,2,13,14)$ & -23.3655 \\
\hline$D(10,2,13,1)$ & -63.0270 \\
\hline$D(10,2,13,12)$ & 162.920 \\
\hline
\end{tabular}




$\begin{array}{lr}\text { D }(10,2,13,14) & 96.0061 \\ \text { D }(11,2,13,1) & 58.0288 \\ \text { D }(11,2,13,12) & -76.0232 \\ \text { D }(11,2,13,14) & -142.9381 \\ \text { D }(3,12,13,1) & 48.0780 \\ \text { D }(3,12,13,2) & 137.6881 \\ \text { D }(3,12,14,7) & -150.4700 \\ \text { D }(3,12,14,8) & -159.6906 \\ \text { D }(1,13,14,7) & 70.0060 \\ \text { D (1,13,14,8) } & 148.1543 \\ \text { D }(2,13,14,7) & -150.5825 \\ \text { D }(2,13,14,8) & -94.2880\end{array}$


Dehydrogenated 1,1-Dimethylhydrazine bisborane [-1H3c] Neutral, ground state, CAM-B3LYP/6-311G(d,p)

Atomic coordinates and value of zero point energy:

$\begin{array}{rrr}0.4503 & 0.85171 & -1.21604 \\ 0.4503 & 0.85166 & 1.21607 \\ -0.74534 & -1.67671 & -0.00001 \\ 1.43327 & 1.31693 & -1.21269 \\ 0.35991 & 0.20445 & -2.08381 \\ -0.33096 & 1.61168 & -1.22349 \\ 1.33432 & -1.7675 & 1.00798 \\ 2.56593 & -0.52456 & -0.00004 \\ 1.33429 & -1.76748 & -1.00804 \\ -3.10432 & -0.87106 & -0.00002 \\ -2.32381 & 1.06091 & 0 . \\ 0.35993 & 0.20436 & 2.08382 \\ 1.43327 & 1.31689 & 1.21273 \\ -0.33096 & 1.61162 & 1.22357 \\ -0.92139 & -0.68173 & -0.00001 \\ 0.33761 & 0.01123 & 0 . \\ 1.5246 & -1.12975 & -0.00003 \\ -2.18514 & -0.11949 & -0.00001\end{array}$

$\mathrm{ZPE}=-242.397432 \mathrm{Ha}$
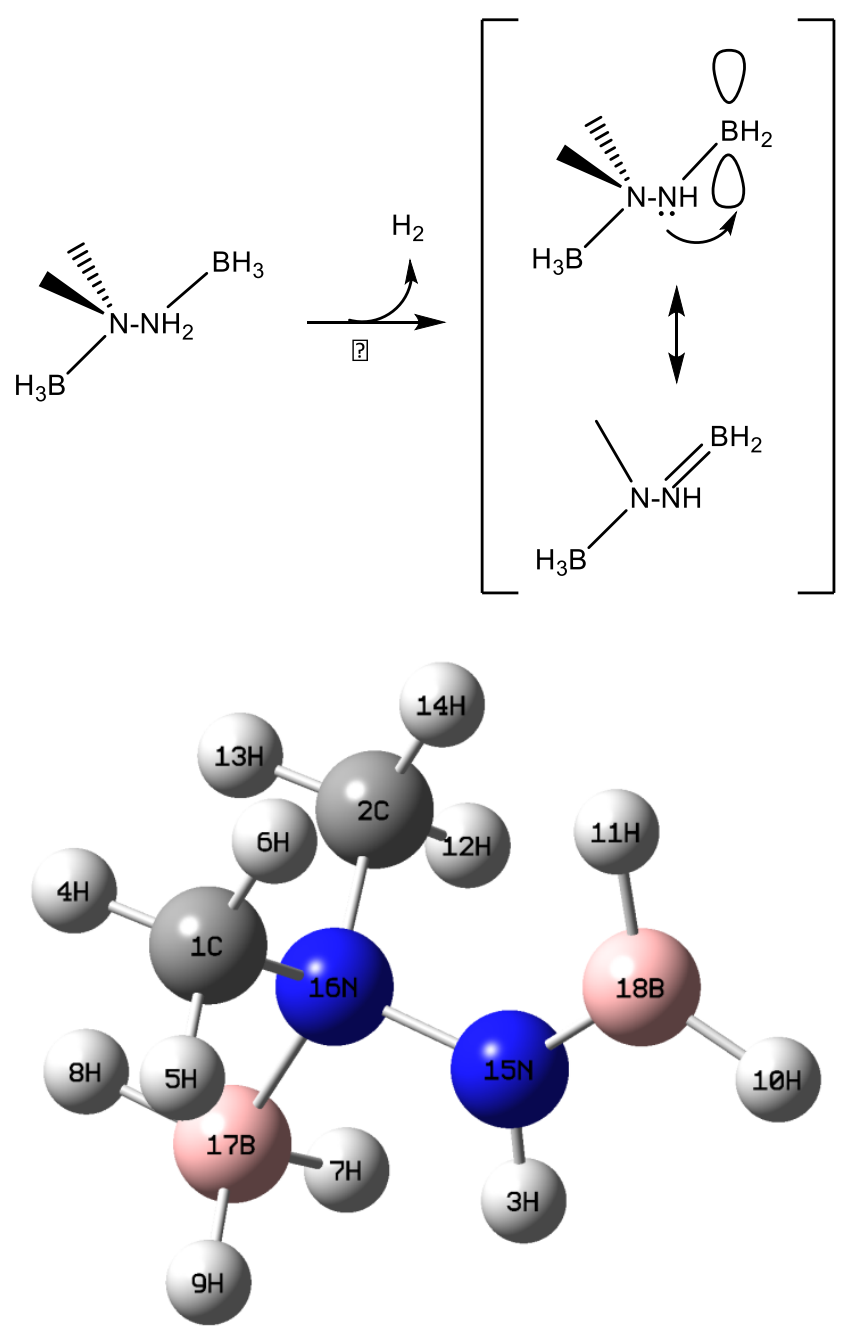
Geometrical parameters [bond distances (R) in $\AA$, angles (A) and dihedral (D) in degrees] :

\begin{tabular}{|c|c|}
\hline $\mathrm{R}(1,4)$ & 1.0875 \\
\hline$R(1,5)$ & 1.0863 \\
\hline$R(1,6)$ & 1.0899 \\
\hline $\mathrm{R}(1,16)$ & 1.4825 \\
\hline$R(2,12)$ & 1.0863 \\
\hline $\mathrm{R}(2,13)$ & 1.0875 \\
\hline$R(2,14)$ & 1.0899 \\
\hline$R(2,16)$ & 1.4825 \\
\hline$R(3,15)$ & 1.0104 \\
\hline$R(7,17)$ & 1.2079 \\
\hline $\mathrm{R}(8,17)$ & 1.2044 \\
\hline$R(9,17)$ & 1.2079 \\
\hline$R(10,18)$ & 1.1873 \\
\hline $\mathrm{R}(11,18)$ & 1.1885 \\
\hline$R(15,16)$ & 1.4371 \\
\hline$R(15,18)$ & 1.3832 \\
\hline$R(16,17)$ & 1.6464 \\
\hline$A(4,1,5)$ & 109.4232 \\
\hline$A(4,1,6)$ & 110.4649 \\
\hline$A(4,1,16)$ & 107.9811 \\
\hline$A(5,1,6)$ & 110.5078 \\
\hline$A(5,1,16)$ & 108.1261 \\
\hline $\mathrm{A}(6,1,16)$ & 110.2685 \\
\hline$A(12,2,13)$ & 109.4236 \\
\hline$A(12,2,14)$ & 110.5077 \\
\hline$A(12,2,16)$ & 108.1261 \\
\hline$A(13,2,14)$ & 110.4648 \\
\hline$A(13,2,16)$ & 107.9810 \\
\hline$A(14,2,16)$ & 110.2684 \\
\hline$A(3,15,16)$ & 108.7942 \\
\hline$A(3,15,18)$ & 124.0183 \\
\hline$A(16,15,18)$ & 127.1876 \\
\hline$A(1,16,2)$ & 110.2224 \\
\hline$A(1,16,15)$ & 109.8740 \\
\hline$A(1,16,17)$ & 109.7590 \\
\hline$A(2,16,15)$ & 109.8737 \\
\hline$A(2,16,17)$ & 109.7590 \\
\hline$A(15,16,17)$ & 107.3041 \\
\hline$A(7,17,8)$ & 113.6728 \\
\hline$A(7,17,9)$ & 113.1312 \\
\hline$A(7,17,16)$ & 104.6139 \\
\hline$A(8,17,9)$ & 113.6725 \\
\hline$A(8,17,16)$ & 105.9682 \\
\hline$A(9,17,16)$ & 104.6138 \\
\hline
\end{tabular}




\begin{tabular}{lr} 
A $(10,18,11)$ & 122.5710 \\
A $(10,18,15)$ & 116.7444 \\
A $(11,18,15)$ & 120.6846 \\
D $(4,1,16,2)$ & 61.8958 \\
D $(4,1,16,15)$ & -176.8803 \\
D $(4,1,16,17)$ & -59.1108 \\
D $(5,1,16,2)$ & -179.8021 \\
D $(5,1,16,15)$ & -58.5782 \\
D $(5,1,16,17)$ & 59.1914 \\
D $(6,1,16,2)$ & -58.8816 \\
D $(6,1,16,15)$ & 62.3422 \\
D $(6,1,16,17)$ & -179.8882 \\
D $(12,2,16,1)$ & 179.8028 \\
D $(12,2,16,15)$ & 58.5788 \\
D $(12,2,16,17)$ & -59.1906 \\
D $(13,2,16,1)$ & -61.8948 \\
D $(13,2,16,15)$ & 176.8812 \\
D $(13,2,16,17)$ & 59.1118 \\
D $(14,2,16,1)$ & 58.8824 \\
D $(14,2,16,15)$ & -62.3416 \\
D $(14,2,16,17)$ & 179.8890 \\
D $(3,15,16,1)$ & 119.2849 \\
D $(3,15,16,2)$ & -119.2821 \\
D (3,15,16,17) & 0.0013 \\
D $(18,15,16,1)$ & -60.7159 \\
D $(18,15,16,2)$ & 60.7170 \\
D $(18,15,16,17)$ & 180.0005 \\
D $(3,15,18,10)$ & -0.0010 \\
D $(3,15,18,11)$ & 179.9994 \\
D $(16,15,18,10)$ & -180.0000 \\
D $(16,15,18,11)$ & 0.0003 \\
D $(1,16,17,7)$ & -178.9473 \\
D $(1,16,17,8)$ & 60.6417 \\
D $(1,16,17,9)$ & -59.7690 \\
D $(2,16,17,7)$ & 59.7671 \\
D $(2,16,17,8)$ & -60.6438 \\
D $(2,16,17,9)$ & 178.9455 \\
D $(15,16,17,7)$ & -59.5899 \\
D $(15,16,17,8)$ & -180.0009 \\
D $(15,16,17,9)$ & 59.5884 \\
\hline
\end{tabular}


Didehydrogenated 1,1-Dimethylhydrazine bisborane [-2H3C]

Neutral, ground state, CAM-B3LYP/6-311G $(d, p)$

Atomic coordinates and value of zero point energy:

$\begin{array}{lccc}\mathrm{C} & 0.77113 & -0.68212 & 1.21785 \\ \mathrm{C} & 0.77113 & -0.68212 & -1.21785 \\ \mathrm{H} & 1.8559 & -0.61478 & 1.2112 \\ \mathrm{H} & 0.37807 & -0.15684 & 2.08316 \\ \mathrm{H} & 0.44177 & -1.72196 & 1.21648 \\ \mathrm{H} & 0.31707 & 2.0614 & -1.01137 \\ \mathrm{H} & 1.97643 & 1.50566 & 0 . \\ \mathrm{H} & 0.31707 & 2.0614 & 1.01137 \\ \mathrm{H} & -3.49883 & 0.02866 & 0 . \\ \mathrm{H} & 0.37807 & -0.15684 & -2.08316 \\ \mathrm{H} & 1.8559 & -0.61478 & -1.2112 \\ \mathrm{H} & 0.44177 & -1.72196 & -1.21648 \\ \mathrm{~N} & -1.10601 & -0.06568 & 0 . \\ \mathrm{N} & 0.26296 & 0.0027 & 0 . \\ \mathrm{B} & 0.77113 & 1.59217 & 0 . \\ \mathrm{B} & -2.33421 & -0.00091 & 0 .\end{array}$

$\mathrm{ZPE}=-241.177176 \mathrm{Ha}$
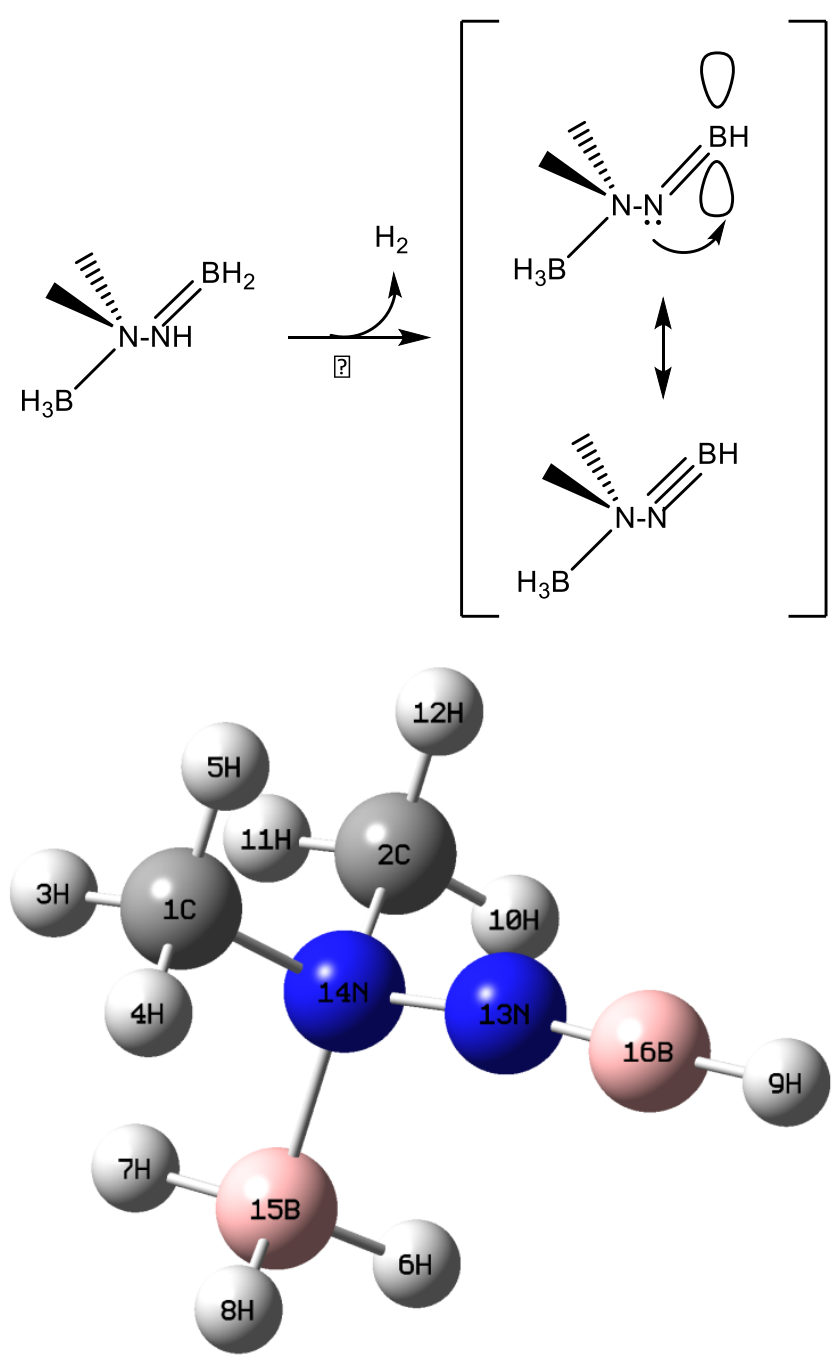
Geometrical parameters [bond distances (R) in $\AA$, angles (A) and dihedral (D) in degrees] :

\begin{tabular}{|c|c|}
\hline$R(1,3)$ & 1.0869 \\
\hline$R(1,4)$ & 1.0859 \\
\hline$R(1,5)$ & 1.0908 \\
\hline$R(1,14)$ & 1.4867 \\
\hline$R(2,10)$ & 1.0859 \\
\hline$R(2,11)$ & 1.0869 \\
\hline$R(2,12)$ & 1.0908 \\
\hline$R(2,14)$ & 1.4867 \\
\hline$R(6,15)$ & 1.2038 \\
\hline$R(7,15)$ & 1.2084 \\
\hline$R(8,15)$ & 1.2038 \\
\hline$R(9,16)$ & 1.1650 \\
\hline$R(13,14)$ & 1.3707 \\
\hline$R(13,16)$ & 1.2299 \\
\hline$R(14,15)$ & 1.6687 \\
\hline$A(3,1,4)$ & 109.6444 \\
\hline$A(3,1,5)$ & 111.1260 \\
\hline$A(3,1,14)$ & 107.9139 \\
\hline$A(4,1,5)$ & 110.6620 \\
\hline$A(4,1,14)$ & 107.8309 \\
\hline$A(5,1,14)$ & 109.5649 \\
\hline$A(10,2,11)$ & 109.6444 \\
\hline$A(10,2,12)$ & 110.6620 \\
\hline$A(10,2,14)$ & 107.8309 \\
\hline$A(11,2,12)$ & 111.1260 \\
\hline$A(11,2,14)$ & 107.9139 \\
\hline$A(12,2,14)$ & 109.5649 \\
\hline$A(1,14,2)$ & 109.9990 \\
\hline$A(1,14,13)$ & 108.5661 \\
\hline$A(1,14,15)$ & 109.5515 \\
\hline$A(2,14,13)$ & 108.5661 \\
\hline$A(2,14,15)$ & 109.5515 \\
\hline$A(13,14,15)$ & 110.5889 \\
\hline$A(6,15,7)$ & 113.8358 \\
\hline$A(6,15,8)$ & 114.3066 \\
\hline$A(6,15,14)$ & 104.8569 \\
\hline$A(7,15,8)$ & 113.8358 \\
\hline$A(7,15,14)$ & 103.6239 \\
\hline$A(8,15,14)$ & 104.8569 \\
\hline $\mathrm{D}(3,1,14,2)$ & 61.779 \\
\hline$D(3,1,14,13)$ & -179.5566 \\
\hline$D(3,1,14,15)$ & -58.696 \\
\hline $\mathrm{D}(4,1,14,2)$ & -179.853 \\
\hline$D(4,1,14,13)$ & -61.189 \\
\hline
\end{tabular}




\begin{tabular}{|c|c|}
\hline$D(4,1,14,15)$ & 59.6707 \\
\hline $\mathrm{D}(5,1,14,2)$ & -59.3439 \\
\hline$D(5,1,14,13)$ & 59.3203 \\
\hline$D(5,1,14,15)$ & -179.8194 \\
\hline$D(10,2,14,1)$ & 179.8538 \\
\hline$D(10,2,14,13)$ & 61.1895 \\
\hline $\mathrm{D}(10,2,14,15)$ & -59.6707 \\
\hline$D(11,2,14,1)$ & -61.7792 \\
\hline$D(11,2,14,13)$ & 179.5566 \\
\hline$D(11,2,14,15)$ & 58.6963 \\
\hline $\mathrm{D}(12,2,14,1)$ & 59.3439 \\
\hline$D(12,2,14,13)$ & -59.3203 \\
\hline$D(12,2,14,15)$ & 179.8194 \\
\hline$D(1,14,15,6)$ & -179.9900 \\
\hline$D(1,14,15,7)$ & 60.3737 \\
\hline $\mathrm{D}(1,14,15,8)$ & -59.2626 \\
\hline$D(2,14,15,6)$ & 59.2626 \\
\hline $\mathrm{D}(2,14,15,7)$ & -60.3737 \\
\hline $\mathrm{D}(2,14,15,8)$ & 179.9900 \\
\hline$D(13,14,15,6)$ & -603.6370 \\
\hline$D(13,14,15,7)$ & 180.0000 \\
\hline$D(13,14,15,8)$ & 60.3637 \\
\hline
\end{tabular}




\section{Transition States}

\section{Dehydrogenation Hydrazine borane $\mathrm{TS}^{\neq}$ Neutral, ground state, CAM-B3LYP/6-311G $(d, p)$}

Atomic coordinates and value of zero point energy:

$\begin{array}{llrr}\mathrm{N} & 1.20483 & 0.30477 & 0.08241 \\ \mathrm{H} & 1.98262 & -0.24559 & 0.42484 \\ \mathrm{~N} & 0.0558 & -0.50631 & -0.03366 \\ \mathrm{H} & 0.13492 & -1.30983 & -0.64405 \\ \mathrm{~B} & -1.31262 & 0.26088 & -0.11483 \\ \mathrm{H} & -2.16692 & -0.42684 & -0.58584 \\ \mathrm{H} & -1.25919 & 1.41889 & -0.39286 \\ \mathrm{H} & -1.5968 & 0.25492 & 1.21469 \\ \mathrm{H} & -0.80658 & -0.29688 & 1.03176 \\ \mathrm{H} & 1.45064 & 0.71174 & -0.81566\end{array}$

$\mathrm{ZPE}=-138.347494 \mathrm{Ha}$

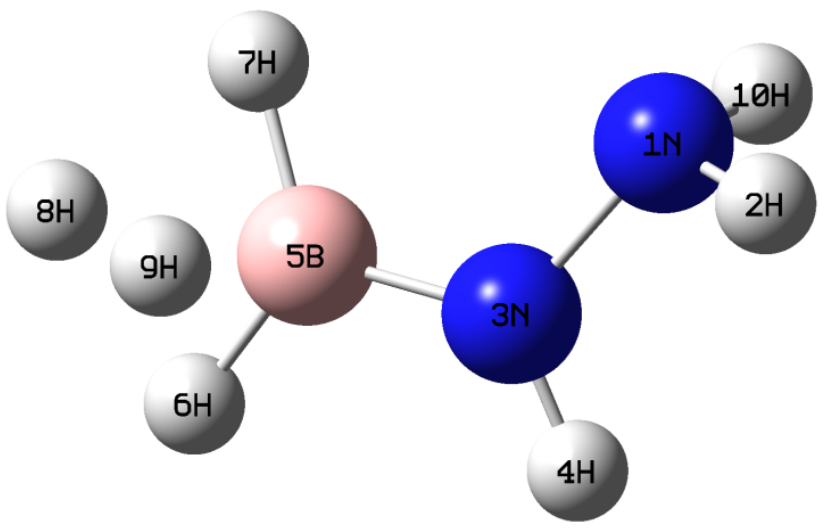

Geometrical parameters [bond distances

(R) in $\AA$, angles

(A) and dihedral

(D) in degrees]:

$\begin{array}{lr}R(1,2) & 1.0125 \\ R(1,3) & 1.4112 \\ R(1,10) & 1.0161 \\ R(3,4) & 1.0122 \\ R(3,5) & 1.5709 \\ R(5,6) & 1.1936 \\ R(5,7) & 1.1921 \\ R(5,8) & 1.3596 \\ R(5,9) & 1.3718 \\ A(2,1,3) & 109.9308 \\ A(2,1,10) & 109.3161 \\ A(3,1,10) & 110.7597 \\ A(1,3,4) & 116.2446 \\ A(1,3,5) & 115.6467\end{array}$




$\begin{array}{lr}\text { A }(4,3,5) & 115.1276 \\ A(3,5,6) & 111.2533 \\ A(3,5,7) & 116.5778 \\ A(3,5,8) & 97.4357 \\ A(3,5,9) & 55.7300 \\ A(6,5,7) & 119.9824 \\ A(6,5,8) & 103.5185 \\ \text { A }(6,5,9) & 111.0754 \\ \text { A }(7,5,8) & 103.9868 \\ \text { A }(7,5,9) & 124.9849 \\ \text { A }(8,5,9) & 42.0952 \\ \text { D }(2,1,3,4) & -58.6764 \\ \text { D }(2,1,3,5) & 161.6922 \\ \text { D }(10,1,3,4) & 62.2363 \\ \text { D }(10,1,3,5) & -77.3950 \\ \text { D }(1,3,5,6) & 159.9939 \\ \text { D }(1,3,5,7) & 17.4197 \\ \text { D }(1,3,5,8) & -92.3257 \\ \text { D }(1,3,5,9) & -98.3597 \\ \text { D }(4,3,5,6) & 19.9094 \\ \text { D }(4,3,5,7) & -122.6648 \\ \text { D }(4,3,5,8) & 127.5898 \\ \text { D (4,3,5,9) } & 121.5558\end{array}$




\section{Dehydrogenation Hydrazine bisborane $\mathrm{TS}^{\neq}$ Neutral, ground state, CAM-B3LYP/6-311G (d,p)}

Atomic coordinates and value of zero point energy:

$\mathrm{H}, 0,0.8224171943,1.9988651728,-0.0378109617$

$\mathrm{H}, 0,0.8787603401,-0.046576186,-0.479252819$

$\mathrm{H}, 0,1.038528478,0.7928600429,2.1382243484$

$\mathrm{H}, 0,-0.3176335155,-0.0627597427,1.7953241242$

$\mathrm{H}, 0,2.6399870402,-0.9491960276,1.3134148235$

$\mathrm{H}, 0,0.9964342928,-1.9951557168,0.7696633429$

$\mathrm{H}, 0,1.3642622231,-1.6369499119,2.7244291181$

$\mathrm{H}, \mathrm{O},-0.9438669859,1.5662539936,-1.2954924446$

$\mathrm{H}, \mathrm{0}, \mathrm{0} .2496284419,2.7968056039,-0.2453652158$

$\mathrm{H}, 0,-1.2623683797,1.9122583693,0.7209041218$

$\mathrm{N}, 0,0.622615743,0.6722188333,0.1841072607$

$\mathrm{N}, 0,0.6403123706,0.1152309801,1.4919124954$

$\mathrm{B}, 0,1.5090736179,-1.2644089775,1.5857957596$

$\mathrm{B}, 0,-0.5232954208,1.6982093867,-0.1917564235$

$\mathrm{ZPE}=-164.959384 \mathrm{Ha}$

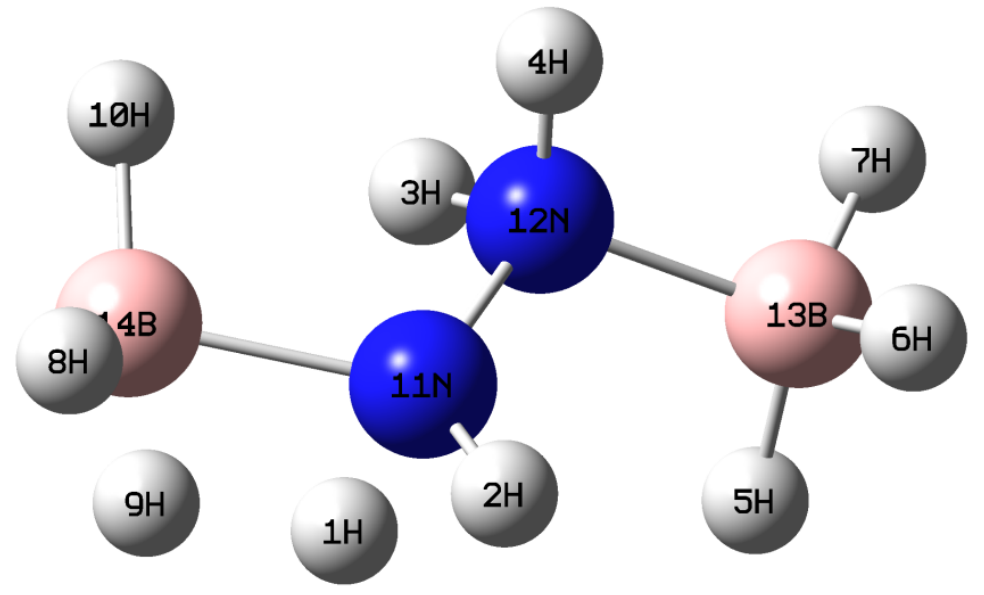

Geometrical parameters [bond distances (R) in $\AA$, angles (A) and dihedral (D) in degrees]:

$\begin{array}{lr}R(1,9) & 1.0039 \\ R(1,11) & 1.3598 \\ R(2,11) & 1.0111 \\ R(3,12) & 1.0176 \\ R(4,12) & 1.0205 \\ R(5,13) & 1.2052 \\ R(6,13) & 1.2095 \\ R(7,13) & 1.2067 \\ R(8,14) & 1.1885 \\ R(9,14) & 1.3443 \\ R(10,14) & 1.1937 \\ R(11,12) & 1.4216 \\ R(12,13) & 1.6331 \\ A(9,1,11) & 136.4959\end{array}$




\begin{tabular}{|c|c|}
\hline$A(1,9,14)$ & 70.7470 \\
\hline$A(1,11,2)$ & 123.3164 \\
\hline$A(1,11,12)$ & 122.0425 \\
\hline$A(2,11,12)$ & 108.7764 \\
\hline$A(3,12,4)$ & 107.1370 \\
\hline$A(3,12,11)$ & 109.1637 \\
\hline$A(3,12,13)$ & 108.5347 \\
\hline$A(4,12,11)$ & 109.2794 \\
\hline$A(4,12,13)$ & 109.5683 \\
\hline$A(11,12,13)$ & 112.9863 \\
\hline$A(5,13,6)$ & 113.7808 \\
\hline$A(5,13,7)$ & 113.9910 \\
\hline$A(5,13,12)$ & 105.3811 \\
\hline$A(6,13,7)$ & 113.5348 \\
\hline$A(6,13,12)$ & 104.2495 \\
\hline$A(7,13,12)$ & 104.5491 \\
\hline$A(8,14,9)$ & 104.9014 \\
\hline$A(8,14,10)$ & 120.7196 \\
\hline$A(9,14,10)$ & 103.8821 \\
\hline$D(11,1,9,14)$ & -28.8160 \\
\hline$D(9,1,11,2)$ & 107.7674 \\
\hline$D(9,1,11,12)$ & -102.2581 \\
\hline$D(1,9,14,8)$ & -115.3387 \\
\hline$D(1,9,14,10)$ & 117.0512 \\
\hline$D(10,9,14,8)$ & 127.6101 \\
\hline$D(1,11,12,3)$ & -13.7684 \\
\hline$D(1,11,12,4)$ & 103.1062 \\
\hline$D(1,11,12,13)$ & -134.6304 \\
\hline$D(2,11,12,3)$ & 140.0223 \\
\hline$D(2,11,12,4)$ & -103.1031 \\
\hline$D(2,11,12,13)$ & 19.1604 \\
\hline$D(3,12,13,5)$ & -58.1690 \\
\hline$D(3,12,13,6)$ & -178.2587 \\
\hline$D(3,12,13,7)$ & 62.2994 \\
\hline$D(4,12,13,5)$ & -174.8487 \\
\hline$D(4,12,13,6)$ & 65.0616 \\
\hline$D(4,12,13,7)$ & -54.3803 \\
\hline$D(11,12,13,5)$ & 63.0497 \\
\hline$D(11,12,13,6)$ & -57.0400 \\
\hline $\mathrm{D}(11,12,13,7)$ & -176.4819 \\
\hline
\end{tabular}




\section{Dehydrogenation Methylhydrazine borane TS $^{\neq}$}

Neutral, ground state, CAM-B3LYP/6-311G (d,p)

Atomic coordinates and value of zero point energy:

$\mathrm{C}, 0,-1.0544746324,0.9883583289,-0.0531584197$

$\mathrm{H}, 0,-1.1579615614,-1.5752512426,0.4970295952$

$\mathrm{H}, \mathrm{0},-0.5123170418,-1.4566022439,-1.0175921754$

$\mathrm{H}, 0,1.1047580687,0.0365403759,1.0859252064$

$\mathrm{H}, 0,-0.7397497293,1.9807400382,0.2673227357$

$\mathrm{H}, \mathrm{O},-1.2828642169,1.0272737143,-1.1284922592$

$\mathrm{H}, \mathrm{O},-1.9689197721,0.7242000983,0.4861506881$

$\mathrm{H}, 0,2.0691744929,0.0661321726,0.9814526953$

$\mathrm{H}, 0,1.6287969646,1.5644988223,-0.2792024816$

$\mathrm{H}, 0,1.9822562296,-0.3380776275,-0.9906470062$

$\mathrm{N}, 0,-0.3239581106,-1.3246118208,-0.0239666173$

$\mathrm{N}, 0,-0.0049628373,0.0272227362,0.231092083$

$\mathrm{B}, 0,1.4648601261,0.386295418,-0.1961752043$

$\mathrm{ZPE}=-177.614138 \mathrm{Ha}$

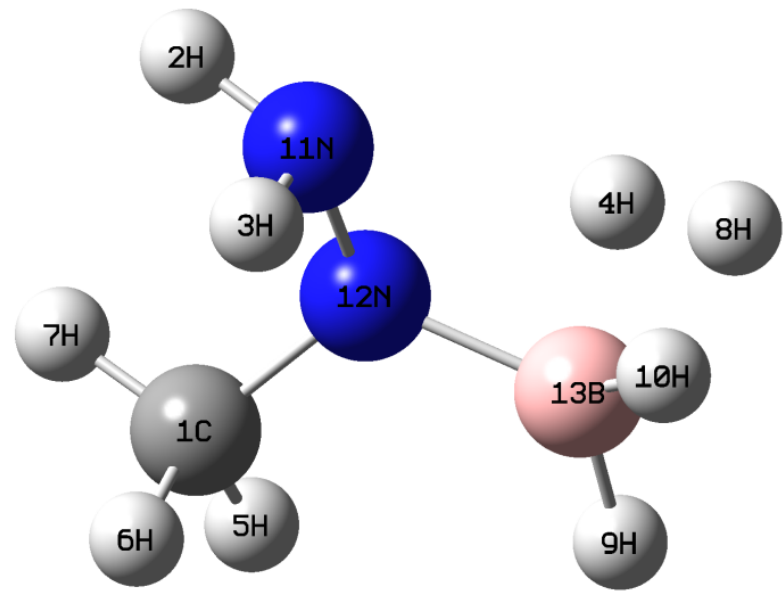

Geometrical parameters [bond distances (R) in $\AA$, angles (A) and dihedral (D) in degrees ] :

$\begin{array}{ll}R(1,5) & 1.0893 \\ R(1,6) & 1.1000 \\ R(1,7) & 1.0940 \\ R(1,12) & 1.4512 \\ R(2,11) & 1.0148 \\ R(3,11) & 1.0199 \\ R(4,13) & 1.3769 \\ R(8,13) & 1.3618 \\ R(9,13) & 1.1924 \\ R(10,13) & 1.1931 \\ R(11,12) & 1.4122 \\ R(12,13) & 1.5722\end{array}$




\begin{tabular}{|c|c|}
\hline$A(5,1,6)$ & 108.3829 \\
\hline$A(5,1,7)$ & 108.4480 \\
\hline$A(5,1,12)$ & 109.6815 \\
\hline$A(6,1,7)$ & 108.4758 \\
\hline$A(6,1,12)$ & 111.4112 \\
\hline$A(7,1,12)$ & 110.3662 \\
\hline$A(2,11,3)$ & 108.4471 \\
\hline$A(2,11,12)$ & 109.2291 \\
\hline$A(3,11,12)$ & 109.9721 \\
\hline$A(1,12,11)$ & 115.8014 \\
\hline$A(1,12,13)$ & 118.1384 \\
\hline$A(11,12,13)$ & 112.3782 \\
\hline$A(4,13,8)$ & 41.5053 \\
\hline$A(4,13,9)$ & 110.5961 \\
\hline$A(4,13,10)$ & 125.3959 \\
\hline$A(4,13,12)$ & 56.2499 \\
\hline$A(8,13,9)$ & 103.3852 \\
\hline$A(8,13,10)$ & 103.9245 \\
\hline$A(8,13,12)$ & 97.2476 \\
\hline$A(9,13,10)$ & 119.5959 \\
\hline$A(9,13,12)$ & 111.9073 \\
\hline$A(10,13,12)$ & 116.5960 \\
\hline$D(5,1,12,11)$ & 174.5666 \\
\hline$D(5,1,12,13)$ & -47.8630 \\
\hline$D(6,1,12,11)$ & -65.4321 \\
\hline$D(6,1,12,13)$ & 72.1382 \\
\hline $\mathrm{D}(7,1,12,11)$ & 55.1425 \\
\hline $\mathrm{D}(7,1,12,13)$ & -167.2871 \\
\hline $\mathrm{D}(2,11,12,1)$ & -56.1460 \\
\hline$D(2,11,12,13)$ & 163.8996 \\
\hline$D(3,11,12,1)$ & 62.7573 \\
\hline $\mathrm{D}(3,11,12,13)$ & -77.1971 \\
\hline$D(1,12,13,4)$ & 125.2816 \\
\hline$D(1,12,13,8)$ & 132.1041 \\
\hline$D(1,12,13,9)$ & 24.4862 \\
\hline$D(1,12,13,10)$ & -118.3258 \\
\hline$D(11,12,13,4)$ & -95.7818 \\
\hline$D(11,12,13,8)$ & -88.9593 \\
\hline$D(11,12,13,9)$ & 163.4228 \\
\hline $\mathrm{D}(11,12,13,10)$ & 20.6108 \\
\hline
\end{tabular}




\section{Dehydrogenation Methylhydrazine bisborane TS $^{\neq}$ Neutral, ground state, CAM-B3LYP/6-311G(d,p)}

$\begin{array}{lrrr}\text { Atomic coordinates and value of zero point energy: } \\ \text { C } & 0,9784973010 & -1,2809527973 & -0,2354759088 \\ \mathrm{H} & -0,4677327012 & 0,3604399858 & 1,5404925546 \\ \mathrm{H} & -1,7418277861 & 0,9871278891 & 0,0796202453 \\ \mathrm{H} & 0,3840526090 & 0,4725285729 & -1,1048711505 \\ \mathrm{H} & 1,8540179411 & -1,3402405274 & -0,8781078891 \\ \mathrm{H} & 1,2524780726 & -1,5977448545 & 0,7686617678 \\ \mathrm{H} & 0,1725528127 & -1,9010006988 & -0,6263360646 \\ \mathrm{H} & 1,2816672913 & 2,2268882135 & 0,3578373051 \\ \mathrm{H} & 2,7028990788 & 0,8862079038 & -0,1611336902 \\ \mathrm{H} & 1,7873016645 & 0,7415847956 & 1,6326497996 \\ \mathrm{H} & -2,6713356880 & -0,8967138255 & 0,8754795588 \\ \mathrm{H} & -2,6533943152 & 0,7654843058 & -0,2616305198 \\ \mathrm{H} & -1,8787369889 & -0,8973931157 & -1,0297591117 \\ \mathrm{~N} & -0,6692588075 & 0,2836515334 & 0,5527134432 \\ \mathrm{~N} & 0,5551651962 & 0,1348392920 & -0,1602167623 \\ \mathrm{~B} & 1,7043051937 & 1,1044420482 & 0,4792412096 \\ \mathrm{~B} & -2,0050576540 & -0,3793079509 & 0,0366997629\end{array}$

$\mathrm{ZPE}=-204.225145 \mathrm{Ha}$

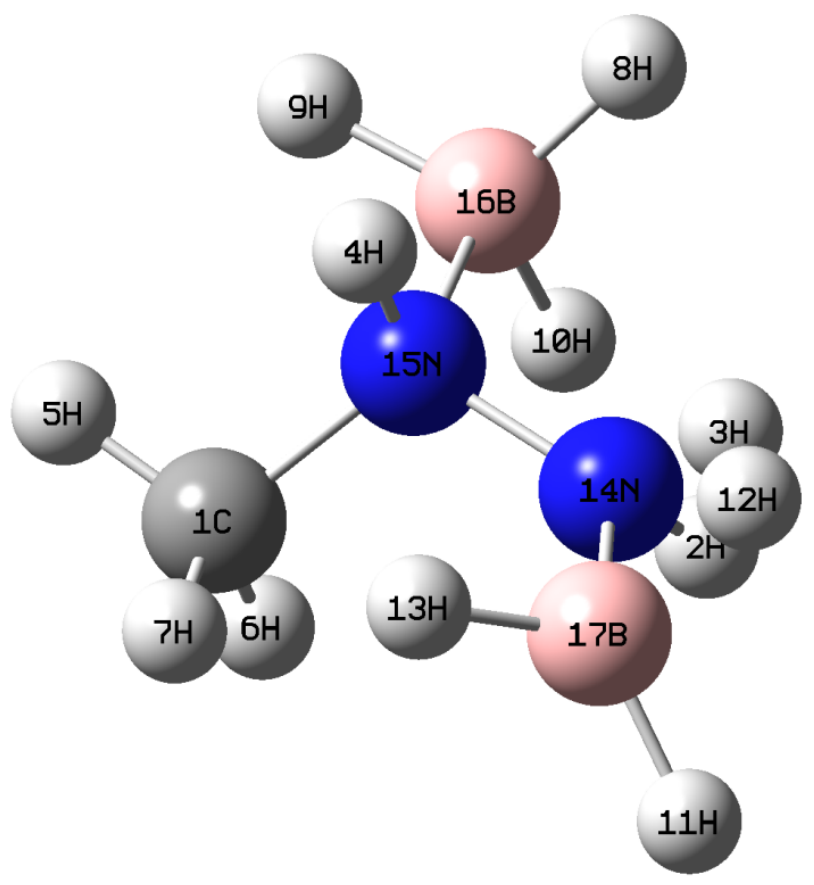


Geometrical parameters (D) in degrees] :

\begin{tabular}{|c|c|}
\hline $\mathrm{R}(1,5)$ & 1,0877 \\
\hline$R(1,6)$ & 1,0880 \\
\hline$R(1,7)$ & 1,0894 \\
\hline $\mathrm{R}(1,15)$ & 1,4796 \\
\hline$R(2,14)$ & 1,0110 \\
\hline $\mathrm{R}(3,12)$ & 0,9983 \\
\hline$R(3,14)$ & 1,3672 \\
\hline $\mathrm{R}(4,15)$ & 1,0177 \\
\hline$R(8,16)$ & 1,2055 \\
\hline$R(9,16)$ & 1,2062 \\
\hline$R(10,16)$ & 1,2120 \\
\hline $\mathrm{R}(11,17)$ & 1,1896 \\
\hline $\mathrm{R}(12,17)$ & 1,3490 \\
\hline$R(13,17)$ & 1,1924 \\
\hline$R(14,15)$ & 1,4247 \\
\hline$R(14,17)$ & 1,5780 \\
\hline$R(15,16)$ & 1,6339 \\
\hline$A(5,1,6)$ & 109,0699 \\
\hline$A(5,1,7)$ & 110,6405 \\
\hline$A(5,1,15)$ & 108,2104 \\
\hline$A(6,1,7)$ & 110,5920 \\
\hline$A(6,1,15)$ & 107,6807 \\
\hline$A(7,1,15)$ & 110,5603 \\
\hline$A(12,3,14)$ & 136,0908 \\
\hline$A(3,12,17)$ & 70,9716 \\
\hline$A(2,14,3)$ & 117,0890 \\
\hline$A(2,14,15)$ & 108,9977 \\
\hline$A(2,14,17)$ & 121,3398 \\
\hline$A(3,14,15)$ & 123,7002 \\
\hline$A(3,14,17)$ & 55,8690 \\
\hline$A(1,15,4)$ & 108,5658 \\
\hline$A(1,15,14)$ & 111,7955 \\
\hline$A(1,15,16)$ & 112,7376 \\
\hline$A(4,15,14)$ & 106,5794 \\
\hline$A(4,15,16)$ & 106,5367 \\
\hline $\mathrm{A}(14,15,16)$ & 110,2814 \\
\hline$A(8,16,9)$ & 113,9065 \\
\hline$A(8,16,10)$ & 113,4914 \\
\hline $\mathrm{A}(8,16,15)$ & 105,4595 \\
\hline$A(9,16,10)$ & 113,2277 \\
\hline$A(9,16,15)$ & 105,4929 \\
\hline $\mathrm{A}(10,16,15)$ & 104,0609 \\
\hline$A(11,17,12)$ & 104,8235 \\
\hline$A(11,17,13)$ & 120,0657 \\
\hline
\end{tabular}

[bond distances (R) in $\AA$, angles (A) and dihedral [bond distances

(n) 


\begin{tabular}{|c|c|}
\hline$A(11,17,14)$ & 115,2311 \\
\hline$A(12,17,13)$ & 102,8174 \\
\hline$A(12,17,14)$ & 97,0435 \\
\hline$A(13,17,14)$ & 112,6648 \\
\hline$L(15,14,17,2,-1)$ & 230,3375 \\
\hline $\mathrm{L}(15,14,17,2,-2)$ & 211,3981 \\
\hline $\mathrm{D}(5,1,15,4)$ & 56,6909 \\
\hline$D(5,1,15,14)$ & 173,9808 \\
\hline$D(5,1,15,16)$ & $-61,1044$ \\
\hline$D(6,1,15,4)$ & 174,4575 \\
\hline$D(6,1,15,14)$ & $-68,2525$ \\
\hline$D(6,1,15,16)$ & 56,6623 \\
\hline $\mathrm{D}(7,1,15,4)$ & $-64,6233$ \\
\hline $\mathrm{D}(7,1,15,14)$ & 52,6666 \\
\hline$D(7,1,15,16)$ & 177,5814 \\
\hline$D(14,3,12,17)$ & $-1,7129$ \\
\hline$D(12,3,14,2)$ & $-109,3699$ \\
\hline$D(12,3,14,15)$ & 109,3128 \\
\hline$D(12,3,14,17)$ & 1,6724 \\
\hline $\mathrm{D}(3,12,17,11)$ & 119,5264 \\
\hline$D(3,12,17,13)$ & $-114,1962$ \\
\hline $\mathrm{D}(3,12,17,14)$ & 1,0369 \\
\hline$D(2,14,15,1)$ & 86,3735 \\
\hline $\mathrm{D}(2,14,15,4)$ & $-155,1476$ \\
\hline $\mathrm{D}(2,14,15,16)$ & $-39,8939$ \\
\hline$D(3,14,15,1)$ & $-129,6770$ \\
\hline$D(3,14,15,4)$ & $-11,1981$ \\
\hline$D(3,14,15,16)$ & 104,0556 \\
\hline $\mathrm{D}(1,15,17,11)$ & 66,7687 \\
\hline $\mathrm{D}(1,15,17,12)$ & $-162,7616$ \\
\hline$D(1,15,17,13)$ & $-60,8307$ \\
\hline $\mathrm{D}(4,15,17,11)$ & 175,2605 \\
\hline$D(4,15,17,12)$ & $-54,2698$ \\
\hline $\mathrm{D}(4,15,17,13)$ & 47,6611 \\
\hline $\mathrm{D}(16,15,17,11)$ & $-70,2685$ \\
\hline$D(16,15,17,12)$ & 60,2012 \\
\hline $\mathrm{D}(16,15,17,13)$ & 162,1321 \\
\hline $\mathrm{D}(2,14,17,11)$ & $-7,5611$ \\
\hline $\mathrm{D}(2,14,17,12)$ & 102,5107 \\
\hline$D(2,14,17,13)$ & $-150,4017$ \\
\hline $\mathrm{D}(3,14,17,11)$ & $-110,9365$ \\
\hline $\mathrm{D}(3,14,17,12)$ & $-0,8647$ \\
\hline$D(3,14,17,13)$ & 106,2229 \\
\hline$D(1,15,16,8)$ & 173,4285 \\
\hline$D(1,15,16,9)$ & 52,5664 \\
\hline$D(1,15,16,10)$ & $-66,8611$ \\
\hline
\end{tabular}


$\mathrm{D}(4,15,16,8)$

$\mathrm{D}(4,15,16,9)$

D $(4,15,16,10)$

$\mathrm{D}(14,15,16,8)$

$\mathrm{D}(14,15,16,9)$

D $(14,15,16,10)$
54,4456

$-66,4164$

174,1560

$-60,8350$

178,3030

58,8755 


\section{Dehydrogenation 1,1-Dimethylhydrazine bisborane TS $^{\neq}$ Neutral, ground state, CAM-B3LYP/6-311G (d,p)}

Atomic coordinates and value of zero point energy:

$\begin{array}{lrrr}\mathrm{C} & 0.60543906840 & 1.14583393000 & 1.72622322210 \\ \mathrm{C} & 0.55791488910 & 1.17571787350 & -0.70304899660 \\ \mathrm{H} & -2.11636675830 & 0.86422471370 & 1.00785660930 \\ \mathrm{H} & -1.62783049690 & -0.45126021020 & -0.47837131170 \\ \mathrm{H} & 1.40593972500 & 1.88148932040 & 1.71059228800 \\ \mathrm{H} & -0.00925215660 & 1.30698671060 & 2.60809738940 \\ \mathrm{H} & 1.01509246040 & 0.13613825800 & 1.72685417780 \\ \mathrm{H} & -1.51346038140 & 2.97689736250 & -0.46337490230 \\ \mathrm{H} & 0.06136451280 & 3.62346857980 & 0.62454110860 \\ \mathrm{H} & -1.57219659470 & 2.88535247160 & 1.55114277160 \\ \mathrm{H} & -1.59779839430 & -1.43987802950 & -0.59861816430 \\ \mathrm{H} & -0.02763233220 & -1.51072503260 & 0.61136072110 \\ \mathrm{H} & -1.93312035890 & -1.65404713020 & 1.37419443250 \\ \mathrm{H} & -0.08965818660 & 1.35658632100 & -1.55716505330 \\ \mathrm{H} & 1.35536609360 & 1.91473482300 & -0.69759377300 \\ \mathrm{H} & 0.97949993420 & 0.17047692120 & -0.74014290260 \\ \mathrm{~N} & -1.33996714920 & 0.43352872580 & 0.52446023530 \\ \mathrm{~N} & -0.24232203980 & 1.35773367940 & 0.52792745190 \\ \mathrm{~B} & -0.88037945870 & 2.87377017170 & 0.55690350570 \\ \mathrm{~B} & -1.15612086580 & -1.13440052960 & 0.63796180020\end{array}$

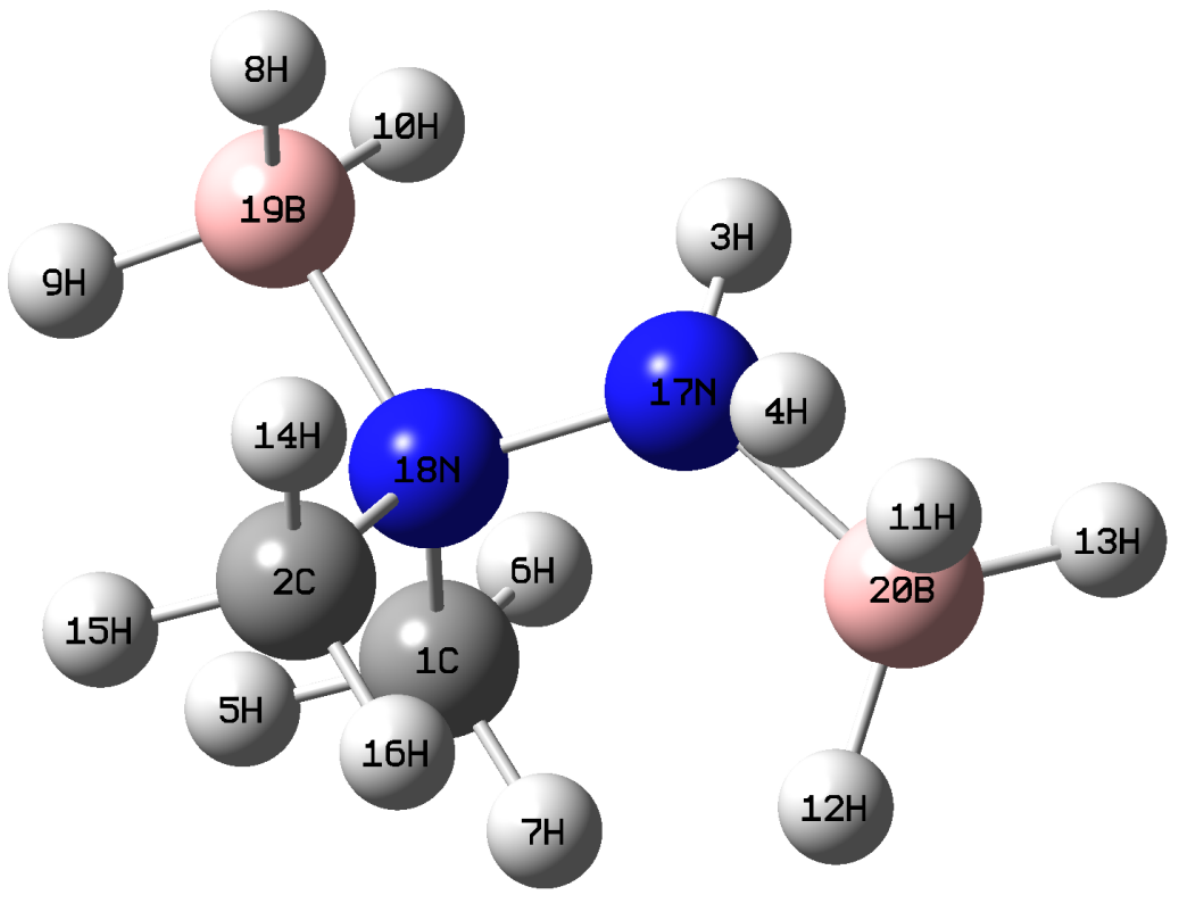


Geometrical parameters (D) in degrees] :

\begin{tabular}{|c|c|}
\hline$R(1,5)$ & 1.0873 \\
\hline$R(1,6)$ & 1.0870 \\
\hline $\mathrm{R}(1,7)$ & 1.0896 \\
\hline $\mathrm{R}(1,18)$ & 1.4831 \\
\hline $\mathrm{R}(2,14)$ & 1.0870 \\
\hline$R(2,15)$ & 1.0872 \\
\hline$R(2,16)$ & 1.0907 \\
\hline $\mathrm{R}(2,18)$ & 1.4795 \\
\hline$R(3,17)$ & 1.0109 \\
\hline$R(4,11)$ & 0.9964 \\
\hline$R(4,17)$ & 1.3680 \\
\hline $\mathrm{R}(8,19)$ & 1.2052 \\
\hline$R(9,19)$ & 1.2056 \\
\hline$R(10,19)$ & 1.2113 \\
\hline$R(11,20)$ & 1.3482 \\
\hline$R(12,20)$ & 1.1899 \\
\hline$R(13,20)$ & 1.1899 \\
\hline $\mathrm{R}(17,18)$ & 1.4349 \\
\hline$R(17,20)$ & 1.5827 \\
\hline $\mathrm{R}(18,19)$ & 1.6451 \\
\hline$A(5,1,6)$ & 109.1288 \\
\hline$A(5,1,7)$ & 110.4978 \\
\hline$A(5,1,18)$ & 108.2135 \\
\hline$A(6,1,7)$ & 110.4577 \\
\hline$A(6,1,18)$ & 108.1245 \\
\hline$A(7,1,18)$ & 110.3509 \\
\hline$A(14,2,15)$ & 109.1350 \\
\hline$A(14,2,16)$ & 110.9101 \\
\hline$A(14,2,18)$ & 108.1240 \\
\hline$A(15,2,16)$ & 110.0675 \\
\hline$A(15,2,18)$ & 107.9946 \\
\hline$A(16,2,18)$ & 110.5337 \\
\hline$A(11,4,17)$ & 136.3764 \\
\hline$A(4,11,20)$ & 70.9949 \\
\hline$A(3,17,4)$ & 117.6766 \\
\hline$A(3,17,18)$ & 108.1756 \\
\hline$A(3,17,20)$ & 118.4878 \\
\hline$A(4,17,18)$ & 125.4027 \\
\hline$A(4,17,20)$ & 55.6869 \\
\hline$A(1,18,2)$ & 110.2127 \\
\hline$A(1,18,17)$ & 110.3157 \\
\hline$A(1,18,19)$ & 109.8248 \\
\hline$A(2,18,17)$ & 109.421 \\
\hline$A(2,18,19)$ & 109.7442 \\
\hline
\end{tabular}

[bond distances (R) in $\AA$, angles (A) and dihedral [bond distances

(a) 


\begin{tabular}{|c|c|}
\hline$A(17,18,19)$ & 107.2719 \\
\hline$A(8,19,9)$ & 113.8674 \\
\hline$A(8,19,10)$ & 113.2065 \\
\hline $\mathrm{A}(8,19,18)$ & 105.5270 \\
\hline$A(9,19,10)$ & 113.2122 \\
\hline$A(9,19,18)$ & 105.7285 \\
\hline$A(10,19,18)$ & 104.1692 \\
\hline$A(11,20,12)$ & 102.6235 \\
\hline$A(11,20,13)$ & 104.7526 \\
\hline$A(11,20,17)$ & 96.9288 \\
\hline$A(12,20,13)$ & 119.6716 \\
\hline$A(12,20,17)$ & 114.9527 \\
\hline$A(13,20,17)$ & 113.6505 \\
\hline $\mathrm{L}(18,17,20,3,-1)$ & 226.6634 \\
\hline$L(18,17,20,3,-2)$ & 213.3183 \\
\hline$D(5,1,18,2)$ & 60.7959 \\
\hline $\mathrm{D}(5,1,18,17)$ & -178.2639 \\
\hline$D(5,1,18,19)$ & -60.2286 \\
\hline $\mathrm{D}(6,1,18,2)$ & 178.8750 \\
\hline$D(6,1,18,17)$ & -60.1848 \\
\hline $\mathrm{D}(6,1,18,19)$ & 57.8505 \\
\hline $\mathrm{D}(7,1,18,2)$ & -60.2171 \\
\hline $\mathrm{D}(7,1,18,17)$ & 60.7231 \\
\hline $\mathrm{D}(7,1,18,19)$ & 178.7584 \\
\hline $\mathrm{D}(14,2,18,1)$ & -178.9496 \\
\hline$D(14,2,18,17)$ & 59.5766 \\
\hline$D(14,2,18,19)$ & -57.8769 \\
\hline$D(15,2,18,1)$ & -60.9826 \\
\hline$D(15,2,18,17)$ & 177.5436 \\
\hline $\mathrm{D}(15,2,18,19)$ & 60.0901 \\
\hline $\mathrm{D}(16,2,18,1)$ & 59.4757 \\
\hline$D(16,2,18,17)$ & -61.9980 \\
\hline $\mathrm{D}(16,2,18,19)$ & -179.4516 \\
\hline$D(17,4,11,20)$ & -1.2380 \\
\hline $\mathrm{D}(11,4,17,3)$ & -105.8977 \\
\hline$D(11,4,17,18)$ & 110.5012 \\
\hline$D(11,4,17,20)$ & 1.2071 \\
\hline$D(4,11,20,12)$ & -116.8190 \\
\hline$D(4,11,20,13)$ & 117.4798 \\
\hline$D(4,11,20,17)$ & 0.7436 \\
\hline $\mathrm{D}(3,17,18,1)$ & 90.3364 \\
\hline$D(3,17,18,2)$ & -148.2520 \\
\hline $\mathrm{D}(3,17,18,19)$ & -29.2564 \\
\hline$D(4,17,18,1)$ & -123.2440 \\
\hline$D(4,17,18,2)$ & -1.8324 \\
\hline $\mathrm{D}(4,17,18,19)$ & 117.163 \\
\hline
\end{tabular}




$\begin{array}{lr}\text { D }(1,18,20,11) & -157.9860 \\ \text { D }(1,18,20,12) & -55.3282 \\ \text { D }(1,18,20,13) & 73.8993 \\ \text { D }(2,18,20,11) & -47.4584 \\ \text { D }(2,18,20,12) & 55.1993 \\ \text { D }(2,18,20,13) & -175.5732 \\ \text { D }(19,18,20,11) & 80.1534 \\ \text { D }(19,18,20,12) & -177.1889 \\ \text { D }(19,18,20,13) & -47.9614 \\ \text { D }(3,17,20,11) & 105.0111 \\ \text { D }(3,17,20,12) & -147.5696 \\ \text { D }(3,17,20,13) & -4.4557 \\ \text { D }(4,17,20,11) & -0.6200 \\ \text { D }(4,17,20,12) & 106.7993 \\ \text { D }(4,17,20,13) & -110.0867 \\ \text { D }(1,18,19,8) & 177.8334 \\ \text { D }(1,18,19,9) & 56.8675 \\ \text { D }(1,18,19,10) & -62.6980 \\ \text { D }(2,18,19,8) & 56.5273 \\ \text { D }(2,18,19,9) & -64.4386 \\ \text { D }(2,18,19,10) & 175.9959 \\ \text { D }(17,18,19,8) & -62.2601 \\ \text { D (17,18,19,9) } & 176.7740 \\ \text { D (17,18,19,10) } & 57.2085\end{array}$




\section{Calculated M0 energies (Tables S1-S7)}

Table S1: Molecular orbital energies for $\mathbf{2 a}, \mathbf{- 1} \mathbf{H} \mathbf{2 a}$ and $\mathbf{- 2 H 2 a}$ : experimental and calculated by Kohn-Sham energies of MOs $\left(-\varepsilon^{K-S}\right)$ and $\triangle S C F+T D-D F T$ at the CAM-B3LYP/6-311G(d,p) level, OVGF, P3 and SAC-Cl. All values in eV. MO's visualizations from Avogadro software.

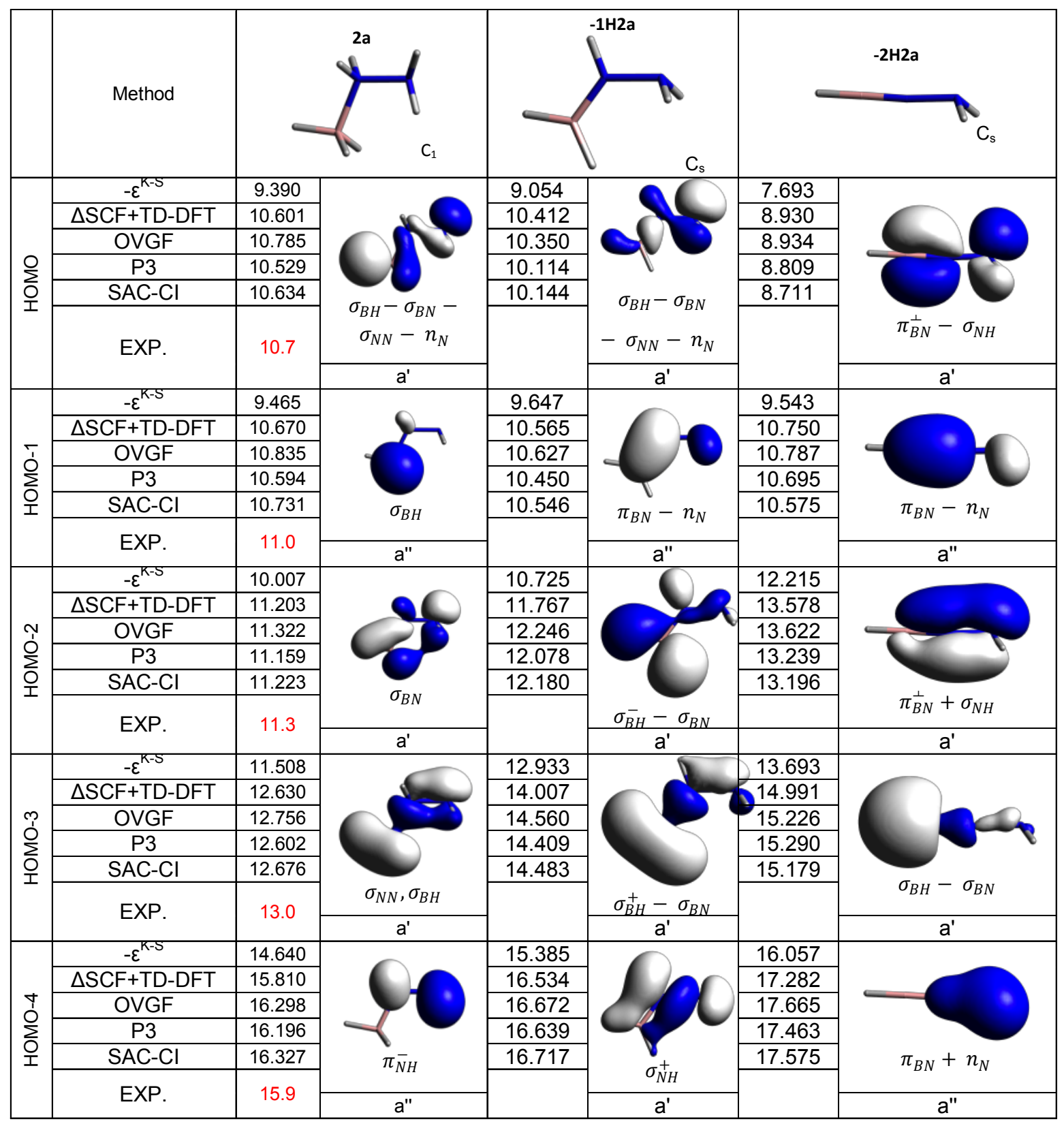


Table S2: Molecular orbital energies for 3a and -1H3a: experimental and calculated by KohnSham energies of MOs $\left(-\varepsilon^{K-S}\right)$ and $\triangle S C F+T D-D F T$ at the CAM-B3LYP/6-311G(d,p) level, OVGF, P3 and SAC-Cl. All values in eV. MO's visualizations from Avogadro software.

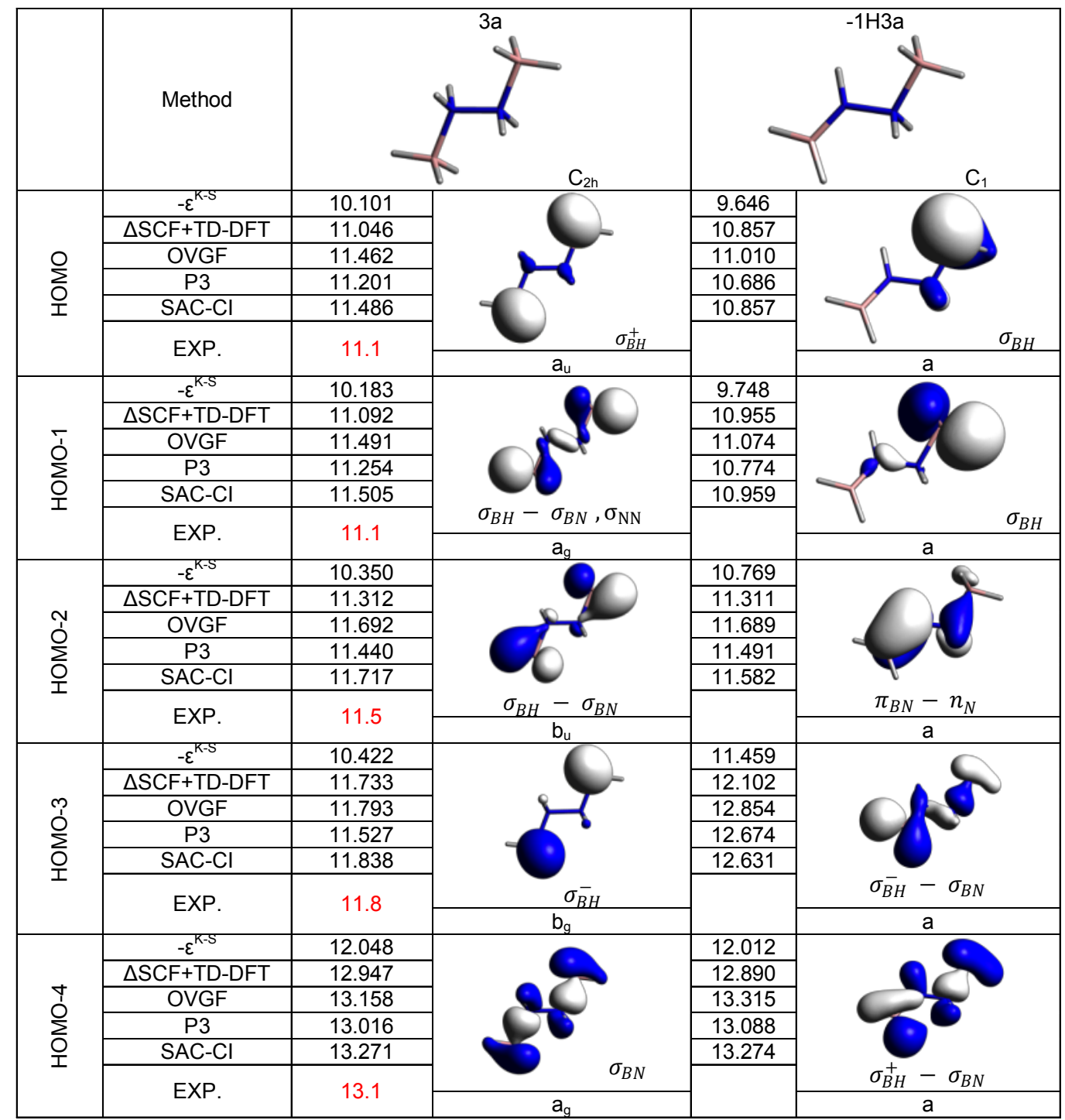


Table S3: Molecular orbital energies for $-\mathbf{2 H 3 a},-3 \mathrm{H} 3 \mathbf{a}$ and $-\mathbf{4 H} 3 \mathrm{a}$ : experimental and calculated by Kohn-Sham energies of MOs $\left(-\varepsilon^{\mathrm{K}-\mathrm{S}}\right)$ and $\triangle \mathrm{SCF}+\mathrm{TD}-\mathrm{DFT}$ at the CAM-B3LYP/6-311G(d,p) level, OVGF, P3 and SAC-Cl. All values in eV. MO's visualizations from Avogadro software.

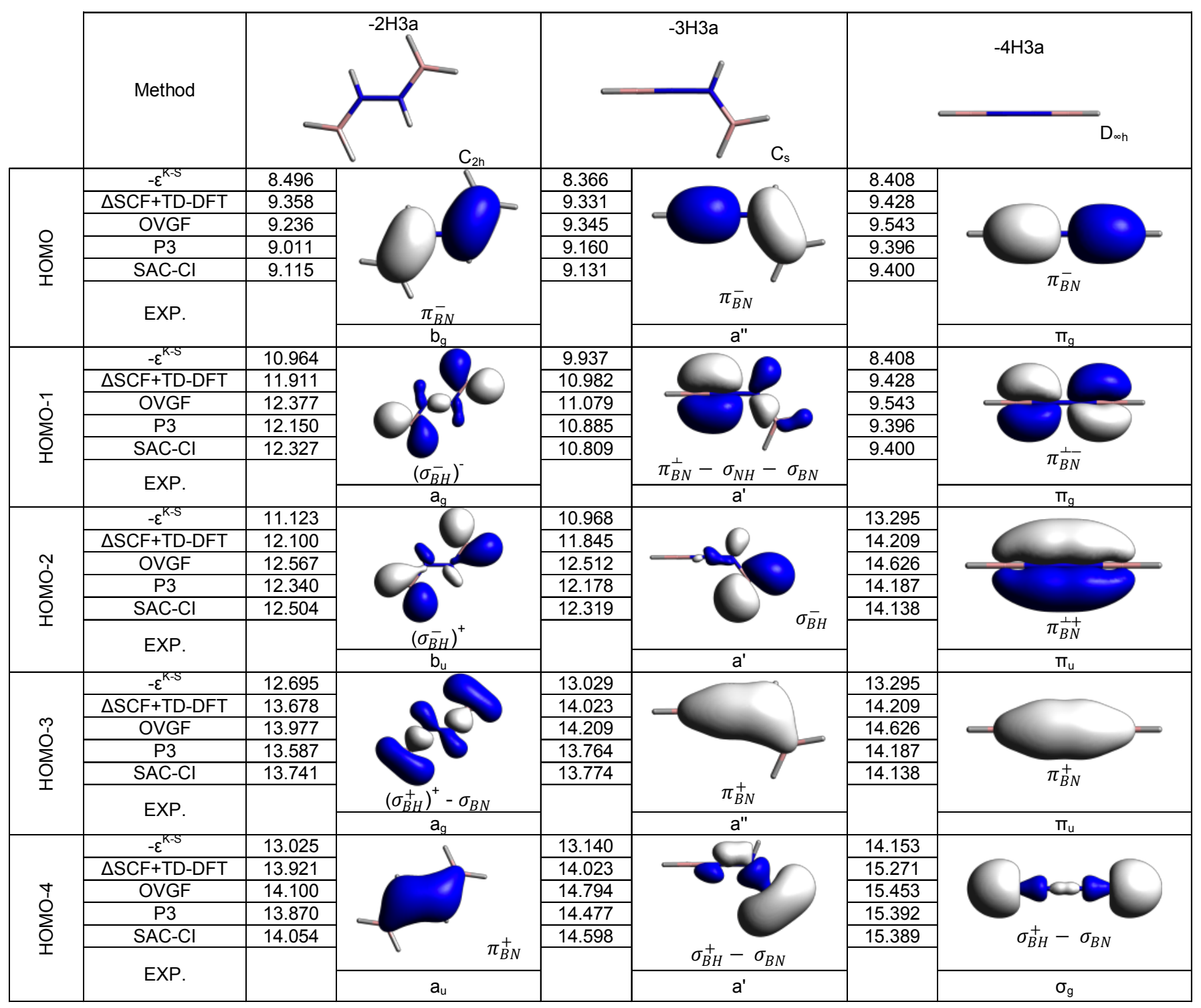


Table S4: Molecular orbital energies for $\mathbf{2 b}$ and $\mathbf{- 1} \mathbf{H} \mathbf{2} \mathbf{b}$ : experimental and calculated by KohnSham energies of MOs $\left(-\varepsilon^{K-S}\right)$ and $\triangle S C F+T D-D F T$ at the CAM-B3LYP/6-311G(d,p) level, OVGF, P3 and SAC-Cl. All values in eV. MO's visualizations from Avogadro software.

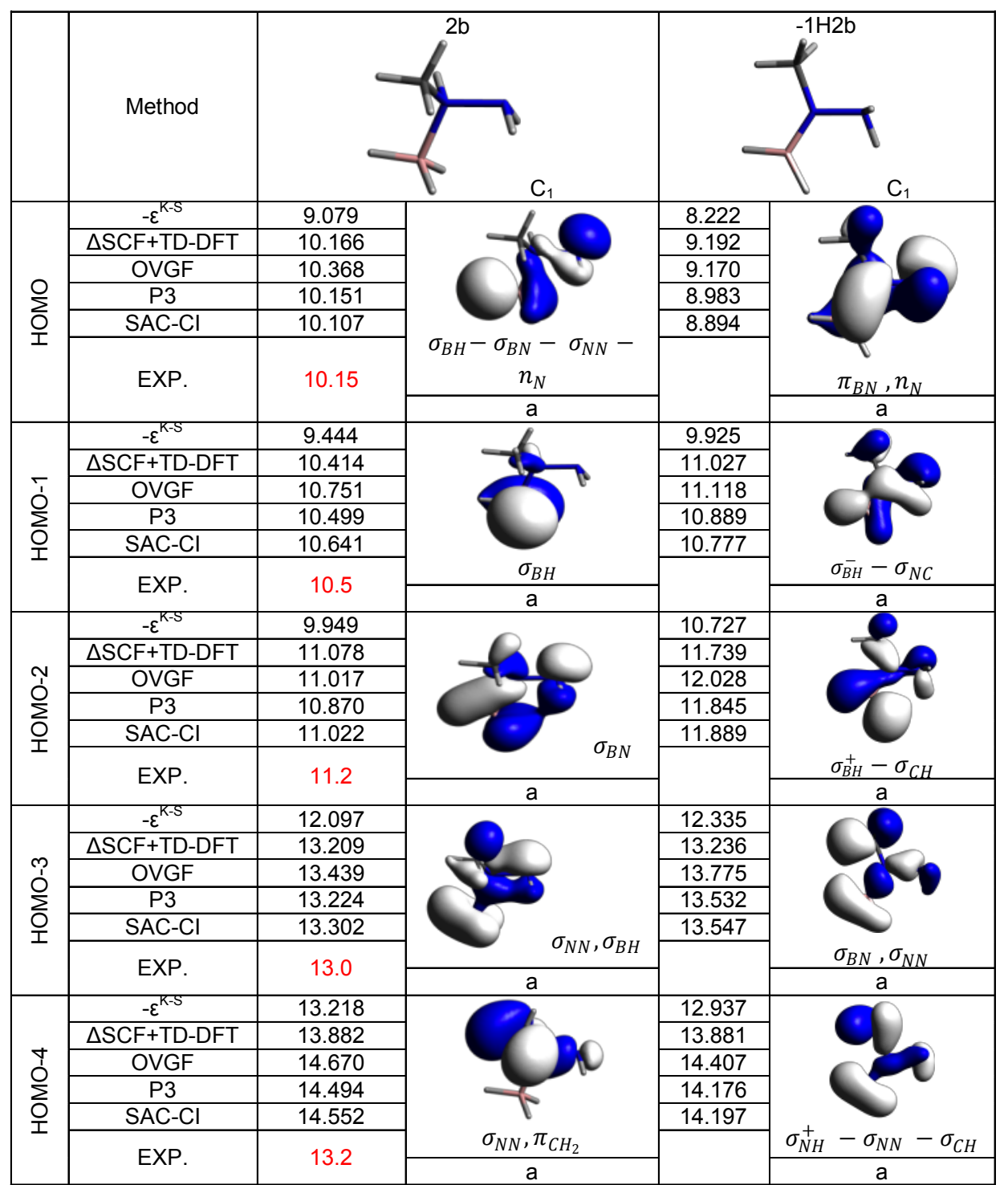


Table S5: Molecular orbital energies for $\mathbf{- 1 H} \mathbf{H b}, \mathbf{- 2 H 3 b}$ and $\mathbf{- 3 H} \mathbf{3 b}$ : experimental and calculated by Kohn-Sham energies of MOs $\left(-\varepsilon^{\mathrm{K}-\mathrm{S}}\right)$ and $\triangle \mathrm{SCF}+\mathrm{TD}-\mathrm{DFT}$ at the CAM-B3LYP/6-311G(d,p) level, OVGF, P3 and SAC-Cl. All values in eV. MO's visualizations from Avogadro software.

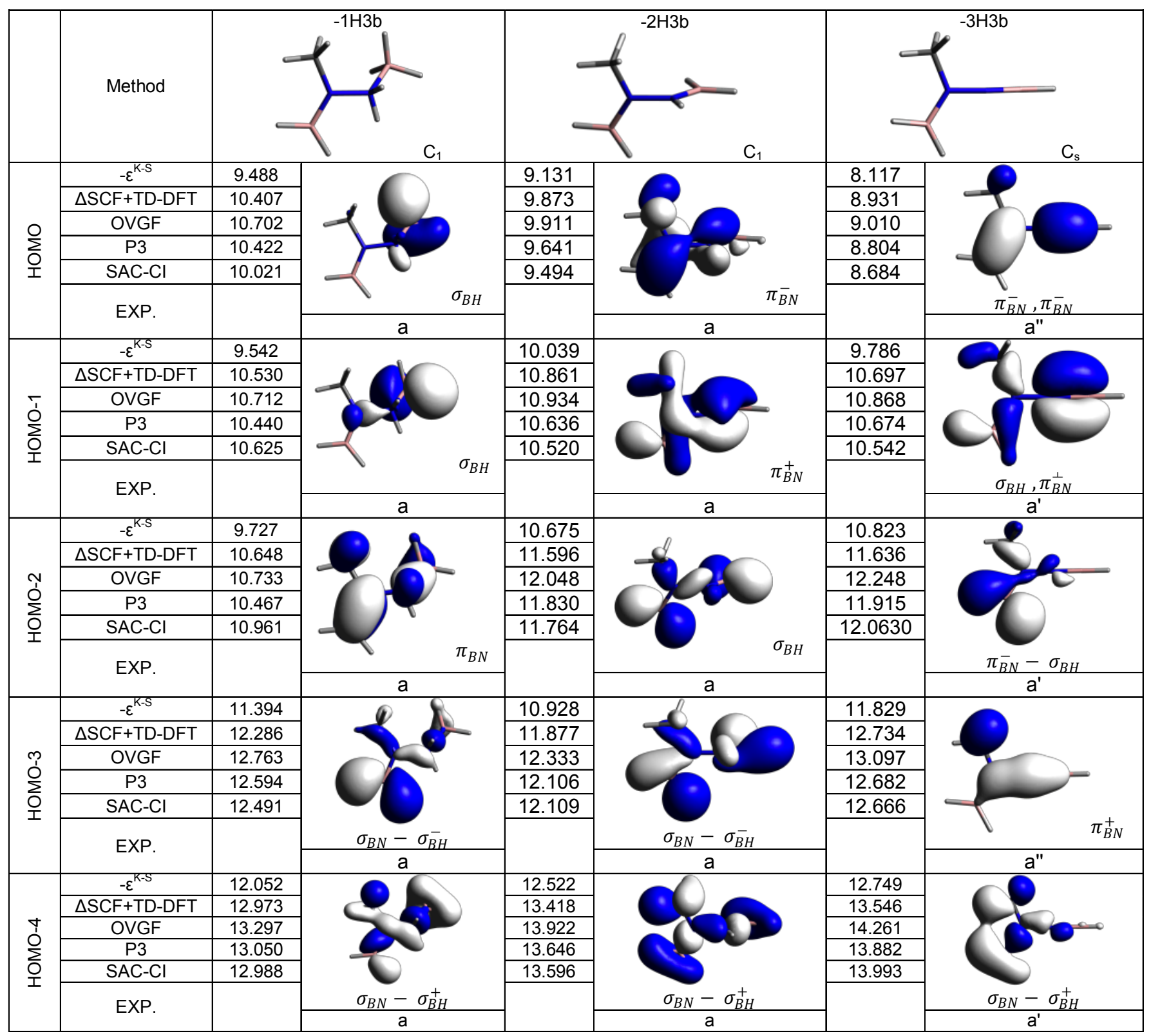


Table S6: Molecular orbital energies for 2c and -1H2c: experimental and calculated by KohnSham energies of MOs $\left(-\varepsilon^{K-S}\right)$ and $\triangle S C F+T D-D F T$ at the CAM-B3LYP/6-311G(d,p) level, OVGF, P3 and SAC-Cl. All values in eV. MO's visualizations from Avogadro software.

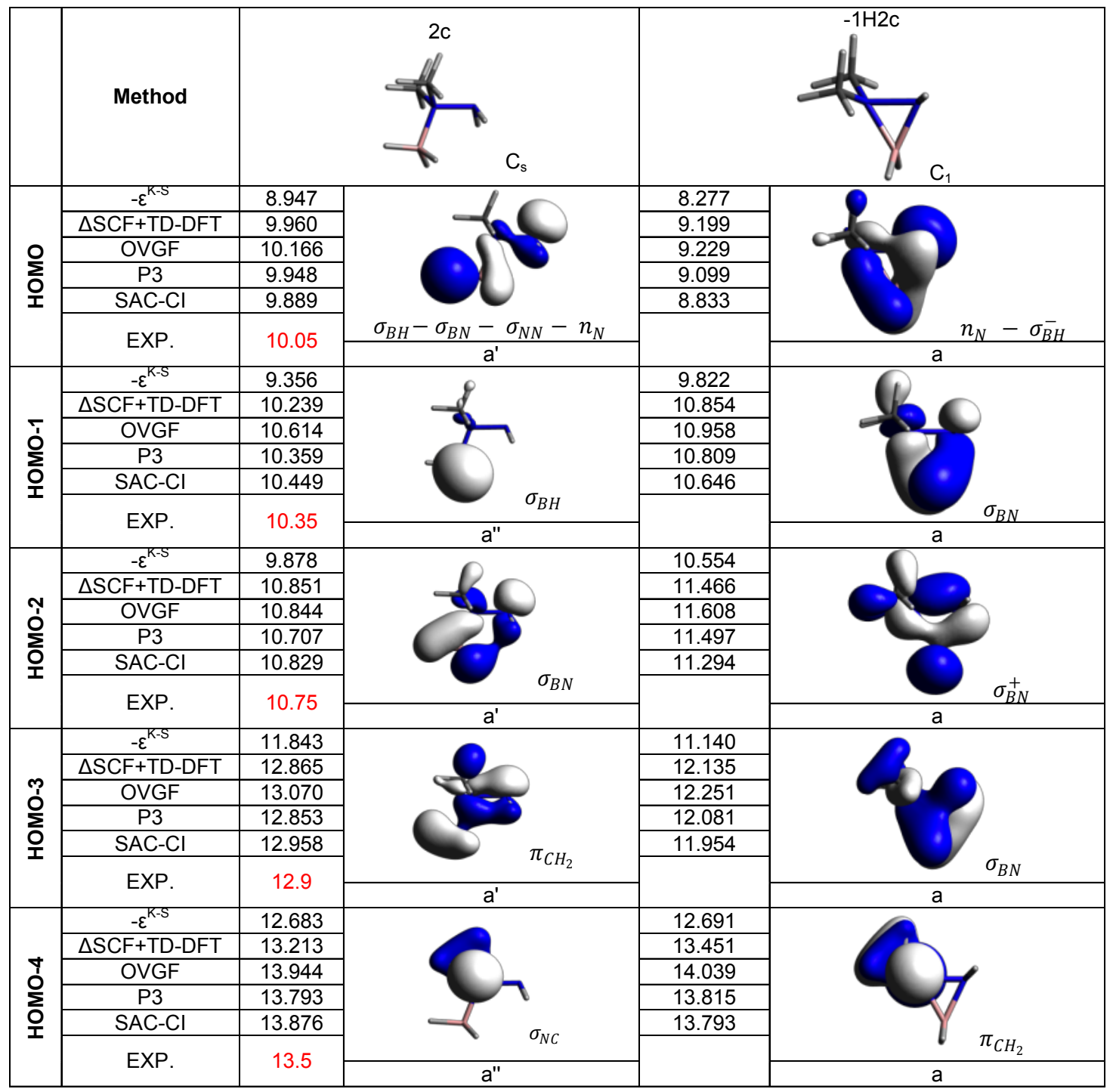


Table S7: Molecular orbital energies for $\mathbf{3 c}, \mathbf{- 1 H 3 c}$ and $\mathbf{- 2 H 3 c}$ : experimental and calculated by Kohn-Sham energies of MOs $\left(-\varepsilon^{K-S}\right)$ and $\triangle S C F+T D-D F T$ at the CAM-B3LYP/6-311G(d,p) level, OVGF, P3 and SAC-Cl. All values in eV. MO's visualizations from Avogadro software.

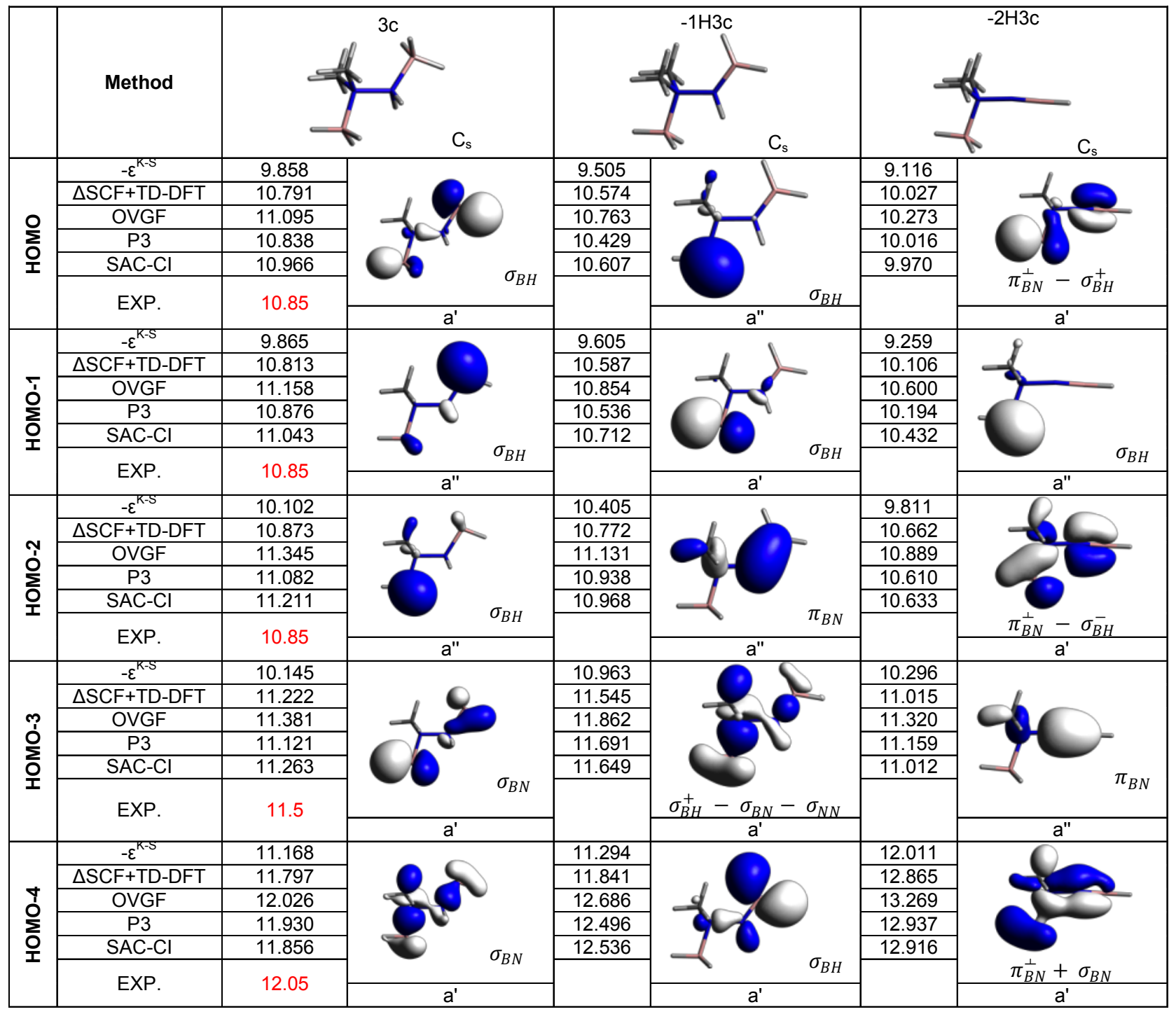


Figures S1-S3

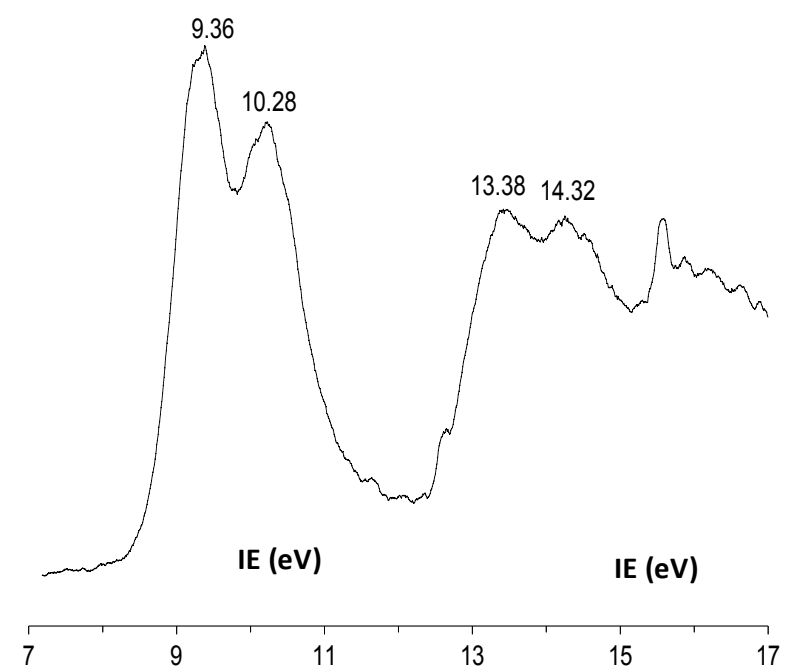

Figure S1: Photoelectron spectrum of methylhydrazine $1 \mathbf{b}\left(70^{\circ} \mathrm{C}\right)$.

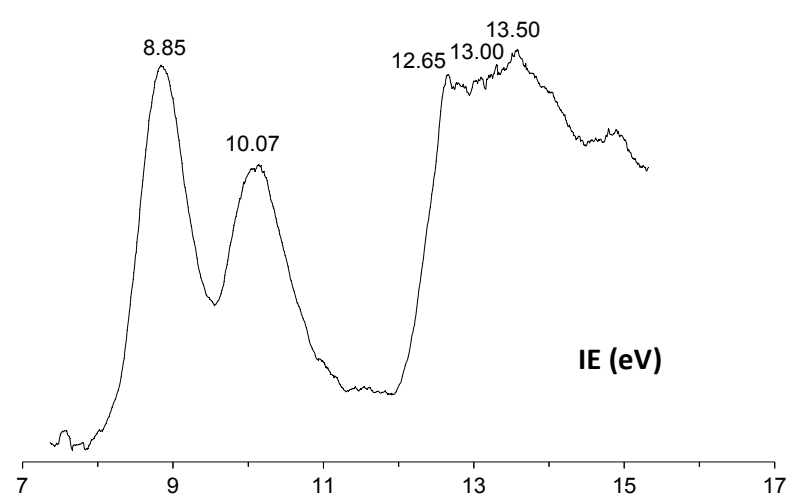

Figure S2: Photoelectron spectrum of 1,1-dimethylhydrazine $1 \mathrm{c}\left(70^{\circ} \mathrm{C}\right)$, all values in $\mathrm{eV}$.

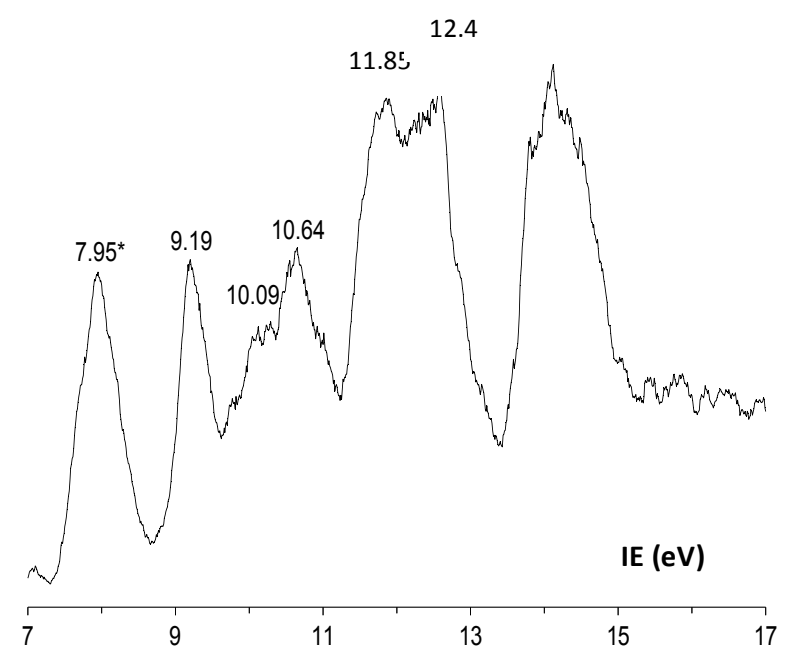

Figure S3: Photoelectron spectrum of phenylhydrazine $1 \mathrm{~d}\left(80^{\circ} \mathrm{C}\right)$ 


\section{Bibliography}

(1) Wrackmeyer, B.; Giumanini, A. G.; Gambi, A.; Verardo, G.; Gilli, G.; Bertolasi, V. Phenylhydrazine-Borane Adduct Characterized in the Solid state and in Solution. Heteroat. Chem. 2002, 13 (4), 366-372. https://doi.org/10.1002/hc.10049. 